\title{
Hidden obstacles in education for students from low socioeconomic backgrounds
}

Citation for published version (APA):

Muskens, M. M. E. (2019). Hidden obstacles in education for students from low socioeconomic backgrounds. [Doctoral Thesis, Maastricht University]. Maastricht University. https://doi.org/10.26481/dis.20191115mm

Document status and date:

Published: 15/11/2019

DOI:

10.26481/dis.20191115mm

Document Version:

Publisher's PDF, also known as Version of record

\section{Please check the document version of this publication:}

- A submitted manuscript is the version of the article upon submission and before peer-review. There can be important differences between the submitted version and the official published version of record.

People interested in the research are advised to contact the author for the final version of the publication, or visit the DOI to the publisher's website.

- The final author version and the galley proof are versions of the publication after peer review.

- The final published version features the final layout of the paper including the volume, issue and page numbers.

Link to publication

\footnotetext{
General rights rights.

- You may freely distribute the URL identifying the publication in the public portal. please follow below link for the End User Agreement:

www.umlib.nl/taverne-license

Take down policy

If you believe that this document breaches copyright please contact us at:

repository@maastrichtuniversity.nl

providing details and we will investigate your claim.
}

Copyright and moral rights for the publications made accessible in the public portal are retained by the authors and/or other copyright owners and it is a condition of accessing publications that users recognise and abide by the legal requirements associated with these

- Users may download and print one copy of any publication from the public portal for the purpose of private study or research.

- You may not further distribute the material or use it for any profit-making activity or commercial gain

If the publication is distributed under the terms of Article $25 \mathrm{fa}$ of the Dutch Copyright Act, indicated by the "Taverne" license above, 


\section{Hidden Obstacles in Education for Students from Low Socioeconomic Backgrounds}

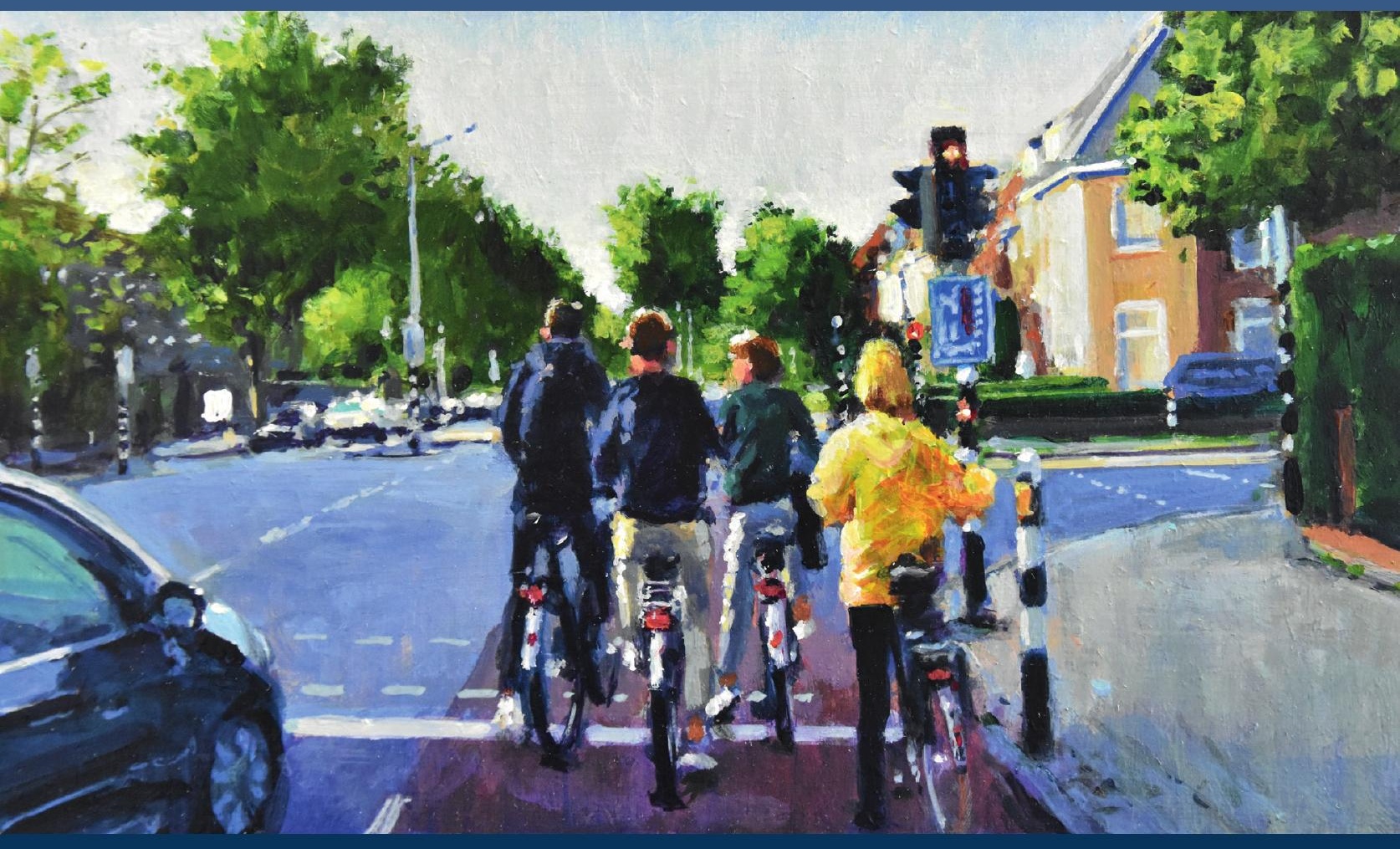



Marjolein Muskens

Hidden Obstacles in Education for Students from Low Socioeconomic Backgrounds 
(C) Marjolein Muskens, 2019

Published by Maastricht University, Postbus 616, 6200 MD Maastricht

Design/Lay-out Proefschriftenbalie.nl

Printed by Ipskamp printing Enschede

Cover picture 'On their way to school' by Diederik Grootjans

ISBN: 978-94-028-1729-4

All rights reserved. No part of this publication may be reproduced, stored in a retrieval system or transmitted in any form or by any means, electronic, mechanical, photocopying, recording or otherwise, without the prior permission in writing from the author. 


\title{
Hidden Obstacles in Education for Students from Low Socioeconomic Backgrounds
}

\author{
Proefschrift \\ ter verkrijging van de graad van doctor aan de Universiteit Maastricht, \\ op gezag van de Rector Magnificus, Prof.dr. Rianne M. Letschert \\ volgens het besluit van het College van Decanen, \\ in het openbaar te verdedigen \\ op vrijdag 15 november 2019 om 12.00 uur
}

door

Marjolein Maria Elisabeth Muskens 


\section{Promotor}

Prof. dr. A.H. Borghans

\section{Copromotor}

Dr. W.E. Frankenhuis (Radboud University)

\section{Beoordelingscommissie}

Prof. dr. M. Levels (voorzitter)

Prof. dr. E.J.P.G. Denessen (Leiden University)

Dr. R. Diris

Dr. P.P.M. Hurks

Prof. dr. M.H.J. Wolbers (Radboud University) 
Seule la vérité peut affronter l'injustice.

La vérité ou bien l'amour

Albert Camus 



\section{Contents}

1 Introduction 11

1.1 Motivation 12

1.2 Aim 13

1.3 Outline and Main Results 13

1.4 References 16

2 Do Economically Disadvantaged Students Perform Better or

Worse on Math Test Items about Money and Social Interaction? 19

2.1 Introduction 21

2.2 Study 1: pilot study $\quad 26$

2.3 Study $2 \quad 29$

2.4 Study $3 \quad 34$

2.5 General discussion $\quad 35$

$\begin{array}{ll}2.6 \text { References } & 40\end{array}$

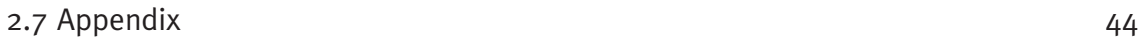

3 The Compensating Role of Personality in the Face of Background

Disadvantage: Predicting School Achievement from Childhood to Adolescence 53

3.1 Introduction $\quad 55$

3.2 Method $\quad 59$

$\begin{array}{ll}3.3 \text { Results } & 61\end{array}$

3.4 Discussion $\quad 66$

$\begin{array}{ll}3.5 \text { References } & 70\end{array}$

4 Economically Disadvantaged Students in Higher Education:

Does Undermatching Improve Integration into College? 75

$\begin{array}{ll}4.1 \text { Introduction } & 77\end{array}$

4.2 Method 80

4.3 Results $\quad 82$

4.4 Discussion $\quad 88$

$\begin{array}{ll}\text { 4.5 References } & 94\end{array}$

$\begin{array}{ll}\text { 4.6 Appendix A } & 98\end{array}$

5 Low-Income Students in Higher Education: Undermatching Predicts

5.1 Introduction 103

$\begin{array}{ll}5.2 \text { Methods } & 106\end{array}$

$\begin{array}{ll}5.3 \text { Results } & 110\end{array}$

$\begin{array}{ll}5.4 \text { Discussion } & 115\end{array}$

5.5 References 121

$\begin{array}{ll}\text { 5.6 Appendix A } & 124\end{array}$ 
6 Conclusions $\quad 127$

6.1 Summary of the main findings 128

6.2 Implications, Knowledge Valorisation, and Recommendations $\quad 130$

$\begin{array}{ll}\text { 6.3 Future Research } & 131\end{array}$

$\begin{array}{ll}6.4 \text { References } & 133\end{array}$

$\begin{array}{ll}\text { Acknowledgements } & 135\end{array}$

$\begin{array}{ll}\text { Curriculum Vitae } & 137\end{array}$ 



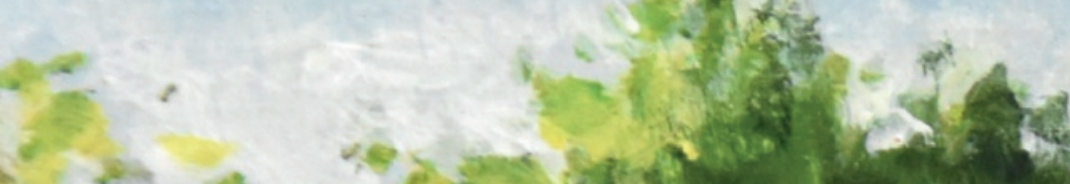

tes is un wat

$2 x-4 x^{2}$

\section{$2=$ \\ SW \\ $x_{2} \cos ^{2}$ \\ $8 x$ \\ $\approx$}

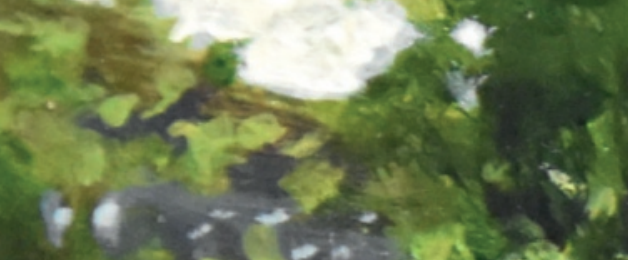

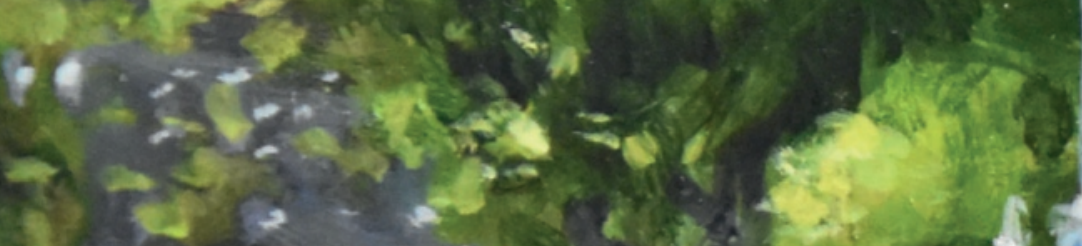

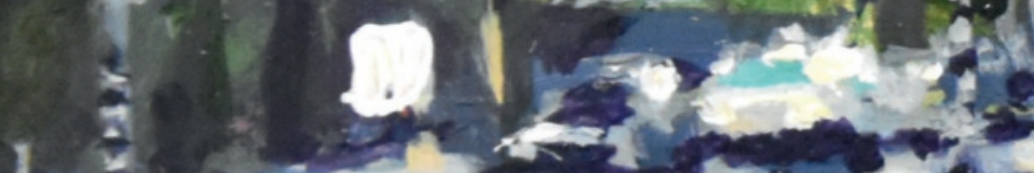

$1 \frac{2}{3}=2$

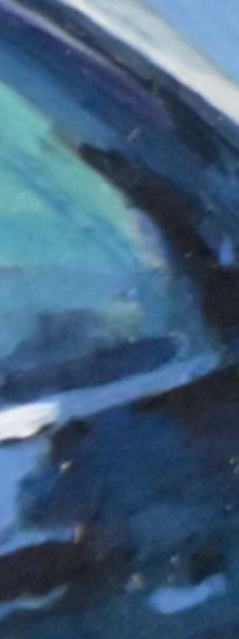




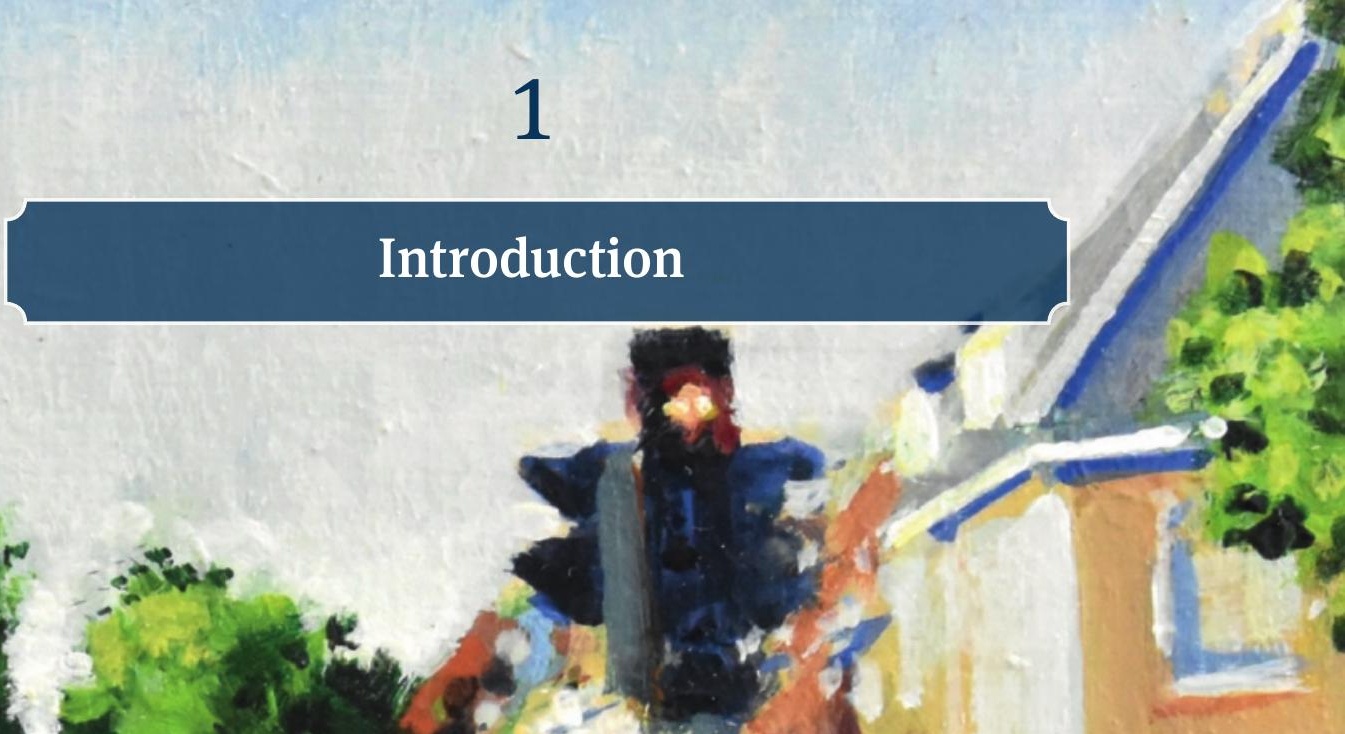

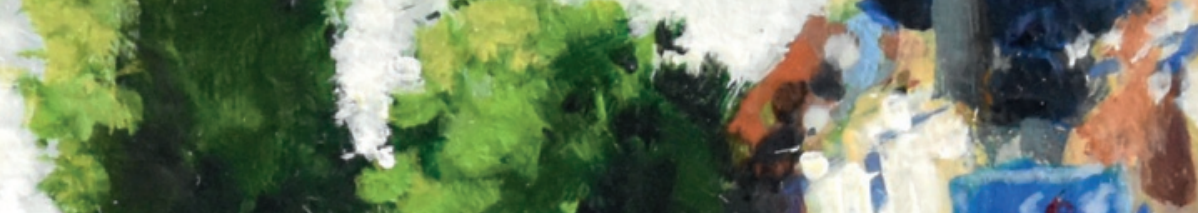

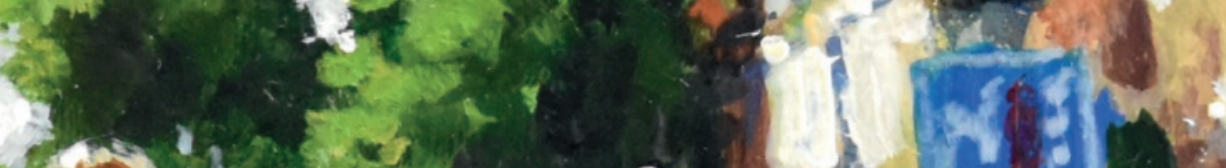

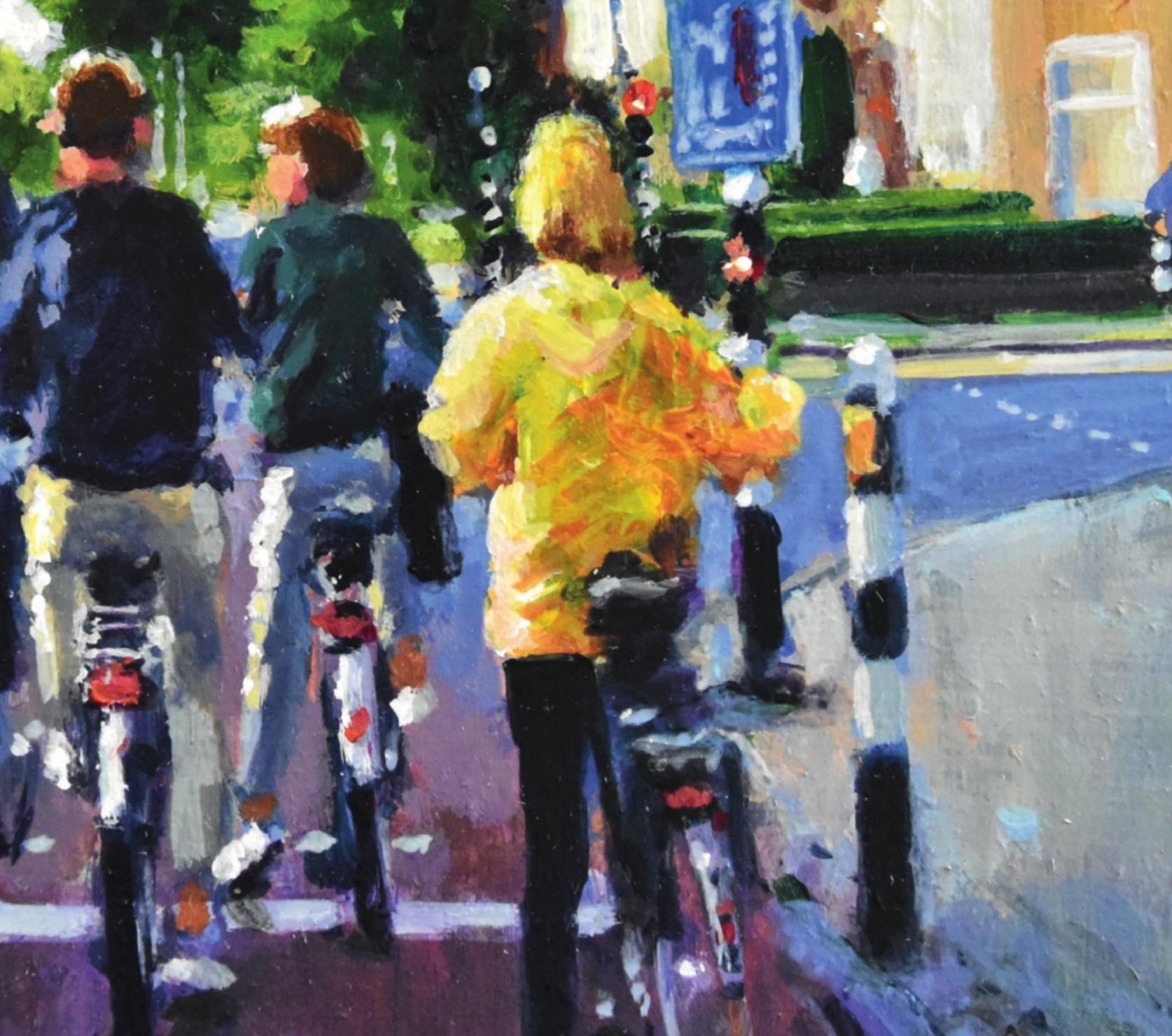




\subsection{Motivation}

Socioeconomic status (SES) is a strong predictor of educational attainment (Sirin, 2005). In the last 50 years, many studies have been added to the research literature on this topic, leading to the conclusion that it is fairly certain that aspects typically associated with low socioeconomic backgrounds, such as experiences of poverty, do have an effect on students' educational attainment (Thomson, 2018). Although many economically disadvantaged students succeed at school, SES predicts significant differences in performance. Advantaged students are likely to outscore their economically disadvantaged peers by large margins (OECD, 2016). Whereas the strength of the predictive value of SES for educational outcomes varies across the world, this relation does exist in each country. Moreover, in the last 15 years, the size of the relation between SES and educational outcomes has, on average, changed little or not at all (OECD, 2016). Notably, while the educational gap between low- and high-SES students continues to exist, the understanding about how this effect is transmitted is far from complete.

The traditional view in psychology and educational science is that poverty and other unbeneficial features of low-SES environments, such as less stimulating home environments, impede children's cognitive and psychosocial development (Mani, Mullainathan, Shafir, \& Zhao, 2013; Vohs, 2013). Whereas there is ample evidence for the detrimental consequences of economic disadvantaged circumstances on development, these effects may not explain all variance in educational outcomes. There may also be a mismatch between demands from contemporary educational systems and skills (e.g., social and cognitive skills) that low-SES students develop in their home environments (Ellis, Bianchi, Griskevicius, \& Frankenhuis, 2017; Rogoff et al., 2017; Valencia, 2010). From the perspective of successful intelligence (Sternberg, 2005), intelligence is the ability to set and realize goals in life given the cultural of socioeconomic context. Whereas basic cognitive skills underlying intelligence may be shared across cultures and contexts, what differs between these contexts is the nature of problems that people come across in their environments. These differences in problems that people encounter in their environments lead to differences in the development of practical knowledge and cognitive and social competences. These differences in competences may in turn impact the capacity to adapt to formal school settings, leading to differences in the ability to adjust and perform (Schliemann \& Carraher, 2002). From this perspective, there may be a mismatch between students' developmental pathways in low-SES environments and demands and features of educational environments.

Educational systems may unintendedly favour high-SES students, because of demands and features that work against low-SES students' ability to learn (Ellis et al., 2017; Rogoff et al., 2017; Sternberg, 2005; Valencia, 2010) or to adapt socially (Stephens, Townsend, \& Dittmann, 2018). For example, standardized math tests are designed to measure students' math ability. However, it has been shown that when children have, because of their socioeconomic circumstances, developed their math skills outside formal school settings, such as on the market, standardized math tests are not able to capture these children's true math ability (Banerjee, Bhattacharjee, Chattopadhyay, \& Ganimian, 2017; Schliemann \& Carraher, 2002). This flaw in tests favours high-SES children, who are more likely to learn math in schools. Moreover, social climates in school settings are known to be tailored toward high-SES values and norms, especially in elite institutions (Jury et al., 2017). 
Low-SES students' cultural orientation is more likely to focus on interdependence, leading to a preference for collaborating with others, and focusing on interests for the group. The predominant cultural orientation in higher education, however, is focused on independence, prioritizing individual goals above others' goals and preferring working independently (Stephens, Fryberg, Markus, Johnson, \& Covarrubias, 2012). Therefore, a cultural mismatch is likely to occur when lowSES students attend higher education, leading to a lower chance to succeed, reinforcing social class-disparities (Stephens et al., 2018).

In order to contribute to the understanding of the remaining gap in educational attainment between students from low- and high SES backgrounds, it is important to gain more insight in features of contemporary educational systems that unintendedly disadvantage students from lowSES backgrounds.

\subsection{Aim}

The goal of this dissertation is to examine empirically hidden and unintended obstacles for students from economically disadvantaged backgrounds in education. In particular, I focus on a mismatch between low-SES students' cognitive and psychosocial development and demands or features in educational settings that may result in lower performance or less well-being. All studies were conducted according the most recent recommendations about research practices, by preregistering research questions data source, definitions, and statistical plans (Wagenmakers, Wetzels, Borsboom, van der Maas, \& Kievit, 2012) because preregistered research is more likely to produce findings that replicate (Nelson, Simmons, \& Simonsohn, 2017). Chapter 2 focusses on the unintended effects of context used to explain mathematical problems in standardized math-tests, on low-SES students' test performance. The aim of chapter 3 is to investigate whether the predictive value of personality traits for educational attainment varies by students' SES. The aim of chapter 4 is to investigate the consequences of attending a less selective college than students' credentials would permit (i.e., undermatching) on low-SES students' academic and social integration in college. The aim of chapter 5 is to provide insight in the long-term consequences of undermatching on students' experiences with the social and academic environment in college. Chapter 6 concludes and summarizes the findings of this thesis, and provides suggestions for future research.

\subsection{Outline and Main Results}

\section{Chapter 2}

In many countries, successful completion of standardized math tests is crucial for academic success. In particular, performance on standardized math tests play an important role in admission and certification procedures. Previous research on fair testing has shown that features of context that is applied to explain mathematical problems can bias the results. For example, the use of academic language in explaining mathematical problems impairs second language learners' performance. In this chapter, we study whether specific context applied in math test items may unintendedly 
bias performance among students who come from low-SES backgrounds. We examine whether context known to be related to recurrent problems in low-SES conditions (i.e., money, food, social interaction) affects low-SES students' performance on math test items, over and above the effect of their 'true math ability' (average math performance) on performance. A large-scale international cohort study of Trends in International Mathematics and Science Studies (TIMSS) including data from 58 countries and from students in grade 6 and 9 (total $(N=5,501,165)$ was used. The results show that math items that include context related to money or social interaction may lead to impaired performance among low-SES students compared to performance among highSES students. These results suggest a need for experimental research of context effects on math items for students from different SES backgrounds. In addition, these findings may have important implications for the design of fair testing at schools.

\section{Chapter 3}

In addition to the well-known positive relation between students' SES and educational outcomes, previous research has shown that certain personality traits, such as Openness, predict educational attainment. This study examines the interplay between students' socioeconomic background and personality traits during childhood, in predicting educational performance in middle adolescence. The aim is to examine whether the predictive value of personality traits varies by students' SES. The outcome variables were test performance (objective measure of students' ability) and educational attainment (determined by teachers' evaluations). These relations were tested with a large and representative longitudinal dataset from the Netherlands $(N=7,409)$, controlling for background characteristics, including intelligence. Regarding students' test performance, results showed that Emotional stability, Openness, and SES have independent positive relations with test performance. In contrast, with regard to students' educational attainment, we also find evidence that Openness is a stronger predictor of educational attainment at lower levels of students' SES. We conclude that for low-SES children, especially Openness may compensate for background disadvantage. We argue that the differential findings for test performance and educational attainment may suggest that the compensating role of personality may work through teachers' evaluations.

\section{Chapter 4}

This chapter investigates barriers in higher education during students' integration into college. It is well documented that students from low-SES backgrounds have a lower chance to attain a degree in highly selective institutions of higher education than high-SES students. Low-SES students are more likely to attend colleges that are less selective than their academic credentials would permit (i.e., undermatching), which has negative implications for their salary and job prospects. In addition, when low-SES students choose to attend highly selective institutions, they are more likely to experience psychological barriers (e.g., a lower sense of belonging) during their integration into college than high-SES students, predicting a higher chance for college dropout. Little is known, however, about the consequences of undermatching for low-SES students' experiences in college. From an academic misalignment perspective, undermatching may lead to less satisfaction because undermatched students' capacities are misaligned with the educational demands and challenges in their institutions. However, from a social misalignment perspective, experiences of social 
mismatch, such as a lower sense of belonging when low-SES students attend highly selective institutions, may be less pronounced in less selective institutions. Consequently, there may be a positive relation between undermatching and experiences with the social environment for lowSES students. Research from a life course perspective shows that these subjective experiences during college may have long-term impact on adolescents' development toward adulthood. This chapter uses a 6-year longitudinal cohort study (Dutch Start Monitor) of 7,305 students in their first year in higher education who are eligible for the most selective institutions (aged 16-24). Results show a replication of the well-documented psychological barriers that low-SES students may experience in highly selective institutions during their first year in college. Specifically, results show that undermatched low-SES students in less selective colleges experience more satisfaction with their social environment than low-SES students in highly selective institutions during their first year in college. Among high-SES students, in contrast, undermatching predicts lower satisfaction with social aspects in college toward the end of the first year in college. However, with regard to academic aspects of college, undermatching does not promote satisfaction, neither for lowSES nor for high-SES students. We conclude that in current higher education settings, low-SES students (but not high-SES students) may benefit from undermatching with regard to their social experiences in college, probably promoting their integration into college during their first year and reducing the risk of college dropout. These findings have implications for policy and interventions during the transition to higher education, aimed at preventing dropout from college and promoting upward social mobility.

\section{Chapter 5}

This chapter examines consequences when students undermatch (i.e., attend higher education institutions that are less selective than their credentials would give access to) for their subjective experiences from the beginning through the final year in college. The current study uses data from a large-scale cohort study among students in the Netherlands (Dutch Student Monitor), including 21,452 respondents. This chapter analyses the association between undermatching, SES, and satisfaction by using propensity score matching (PSM). The results indicated, both for low- and high-SES students, a negative relation between undermatching and satisfaction with the social and academic environment. The negative relation between undermatching and satisfaction increases toward the final stage in higher education, especially for low-SES students. This lowered satisfaction in the later years in higher education implies that the negative consequences of undermatching become more pronounced after students have become more integrated in their colleges. These findings have implications for the understanding about students' development in relation to undermatching, and for policy and interventions aimed at promoting social mobility. 


\subsection{References}

Banerjee, A. V., Bhattacharjee, S., Chattopadhyay, R., \& Ganimian, A. J. (2017). The Untapped Math Skills of Working Children in India: Evidence, Possible Explanations, and Implications. Unpublished manuscript.

Ellis, B. J., Bianchi, J., Griskevicius, V., \& Frankenhuis, W. E. (2017). Beyond risk and protective factors: An adaptation-based approach to resilience. Perspectives on Psychological Science, 12(4), 561-587.

Jury, M., Smeding, A., Stephens, N. M., Nelson, J. E., Aelenei, C., \& Darnon, C. (2017). The experience of low-SES students in higher education: Psychological barriers to success and interventions to reduce social-class inequality. Journal of Social Issues, 73(1), 23-41.

Mani, A., Mullainathan, S., Shafir, E., \& Zhao, J. (2013). Poverty impedes cognitive function. Science, 341, 976-980.

Nelson, L. D., Simmons, J., \& Simonsohn, U. (2017). Psychology’s renaissance. Annual Review of Psychology, 69, 511-534.

Organisation for Economic Co-operation and Development. (2016). PISA 2015 Results: Excellence and Equity in Education. OECD, Paris.

Rogoff, B., Coppens, A. D., Alcalá, L., Aceves-Azuara, I., Ruvalcaba, O., López, A., \& Dayton, A. (2017). Noticing learners' strengths through cultural research. Perspectives on Psychological Science, 12, 876-888.

Schliemann, A. D., \& Carraher, D. W. (2002). The evolution of mathematical reasoning: Everyday versus idealized understandings. Developmental Review, 22(2), 242-266.

Sirin, S. R. (2005). Socioeconomic status and academic achievement: A meta-analytic review of research. Review of educational research, 75(3), 417-453.

Sternberg, R. J. (2005). The theory of successful intelligence. Interamerican Journal of Psychology, 39(2), 189-202.

Stephens, N. M., Fryberg, S. A., Markus, H. R., Johnson, C. S., \& Covarrubias, R. (2012). Unseen disadvantage: How American universities' focus on independence undermines the academic performance of first-generation college students. Journal of Personality and Social Psychology, 102(6), 1178-1197.

Stephens, N. M., Townsend, S. S., \& Dittmann, A. G. (2018). Social-class disparities in higher education and professional workplaces: The role of cultural mismatch. Current Directions in Psychological Science, 28(1), 67-73.

Thomson, S. (2018). Achievement at school and socioeconomic background-an educational perspective. Npj Science of Learning, 5(3), 1-2.

Valencia, R. R. (2010). Dismantling contemporary deficit thinking: Educational thought and practice. New York, NY: Routledge.

Wagenmakers, E. J., Wetzels, R., Borsboom, D., van der Maas, H. J. L., \& Kievit, R. A. (2012). An agenda for purely confirmatory research. Perspectives on Psychological Science, 7, 632-638.

Vohs, K. D. (2013). The poor's poor mental power. Science, 341, 969-970. 



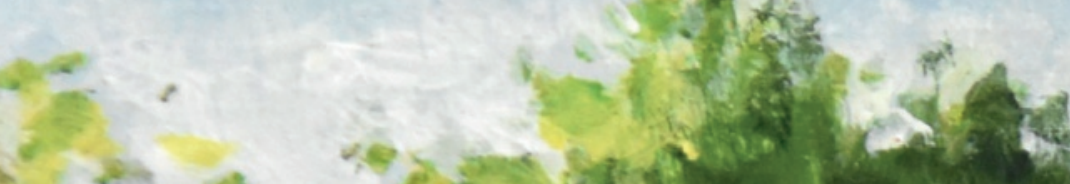

tes is un wat

$2 x-4 x^{2}$

\section{$2=$ \\ SW \\ $x_{2} \cos ^{2}$ \\ $8 x$ \\ $\approx$}

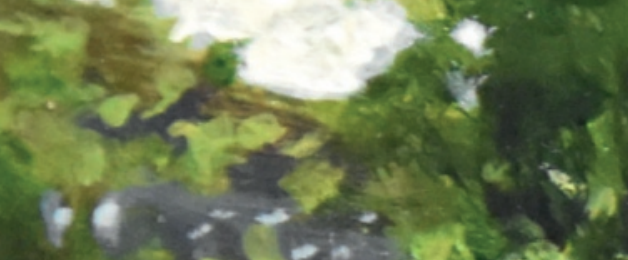

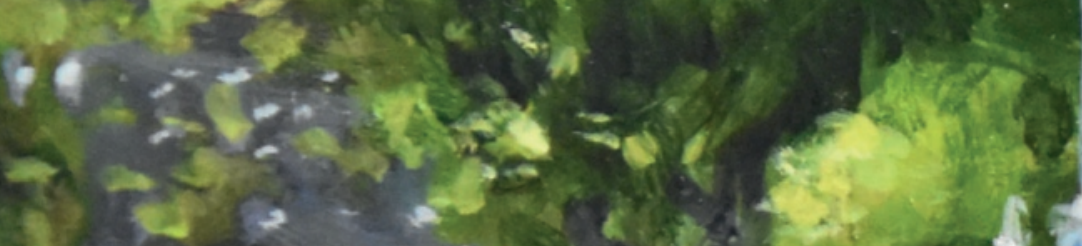

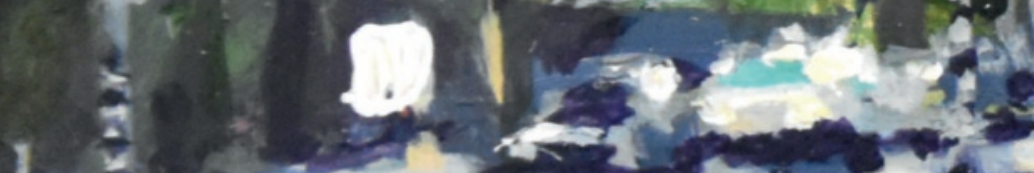

$1 \frac{2}{3}=2$

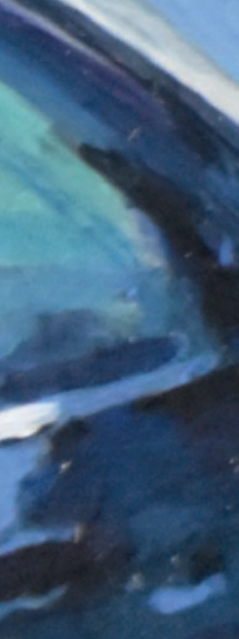




\section{Do Economically Disadvantaged Students Perform Better or Worse on Math Test Items about Money and Social Interaction? ${ }^{1}$}

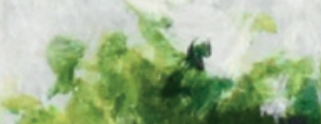

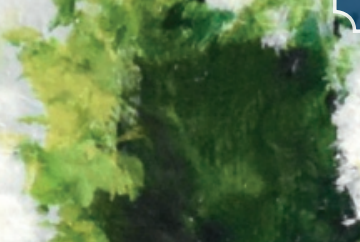




\begin{abstract}
Socioeconomic status (SES) is highly correlated with academic performance. In many countries, successful completion of standardized math tests is crucial for academic success. In this chapter, we study whether context applied in math test items may unintendedly bias performance among students who come from low socioeconomic (SES) backgrounds. In a large scale international cohort study of Trends in International Mathematics and Science Studies (TIMSS) including data from 58 countries and from students in grade 4 and 8 (total $N=5,501,165$ ), we examine whether context known to be related to recurrent problems in low-SES conditions (i.e., money, food, social interaction) affects low-SES students' performance on math test items, over and above the effect of their true math ability (average math performance), on performance. Our results show that math items that include money or social interaction examples, lead to impaired performance of lowSES students. The effects are considerable: students from low-SES backgrounds have on average 16 to $18 \%$ lower chance of giving the correct answer when items contain this relevant context, given their overall performance on the test. This finding has implications for the design of fair testing at schools to avoid reinforcement of the SES gap. Our work suggests a need for experimental research of context effects on math items for students from different SES backgrounds.
\end{abstract}




\subsection{Introduction}

Despite policy-efforts to reduce the gap in academic achievement between students from low- and high- socioeconomic status (SES), background disadvantage is still highly predictive for lower academic performance (Banerjee, 2016; Sirin, 2005). In many countries, standardized math tests co-determine certification and admission procedures for secondary and tertiary education, and passing these tests successfully is crucial for academic achievement. Standardized math tests are designed to measure the math ability of the examinee. However, test items may carry demands with them other than those intended by the test developer (Scheuneman \& Grima, 1997; Warne, Yoon, \& Price, 2014). When personal characteristics, such as gender or socioeconomic status (SES), systematically improve or reduce performance on a test item among people with the same underlying true ability, this leads to biased test results (Walker, 2011). Obviously, fairness in testing is important in reducing the gap between low- and high-SES students and, as stated by Standards for Educational and Psychological Testing (AERA, APA, \& NCME, 2014), minimalizing irrelevant features in tests that might impede performance of low-SES students is of special importance in maximizing accessibility of education.

It is well-documented that complexity of language applied in math test-items can unintendedly impair low-SES students' performance (Abedi \& Lord, 2001; Carpenter, 1980; Haag, Heppt, Stanat, Kuhl, \& Pant, 2013). Less is known about the role of the specific context in test-items that is applied to explain mathematical problems. In this paper, we focus on this context, and examine whether context may unintendedly influence performance among low-SES students. More specifically, in a large-scale international cohort study $(\mathrm{N}=5,501,165)$ of Trends in International Mathematics and Science Studies (TIMSS), we examine whether context applied in math test items related to topics known to be salient in low-SES conditions (i.e., money, food, social interaction, as we will explain below) influences low-SES students' performance, over and above the effect of their math ability.

\section{Socioeconomic status and math performance}

Students from low-SES backgrounds perform, on average, lower on math tests than highSES students (Banerjee, 2016; Sirin, 2005). The predominant view in psychology and education research is that this difference can be explained, at least in part, by deficits in cognitive abilities among low-SES students, which may result from consequences of adverse circumstances during childhood or suboptimal opportunities in education (e.g., Heberle \& Carter, 2015; Mani, Mullainathan, Shafir, \& Zhao, 2013).

However, lower performance on standardized tests among low-SES students may reflect not only deficits in cognitive abilities per se, but also a mismatch between cognitive abilities that have developed in interaction with their home environment and demands of tests in the school environment (Ellis, Bianchi, Griskevicius, \& Frankenhuis, 2017; see also Frankenhuis \& de Weerth, 2013; Rogoff et al., 2017; Sternberg, 2014; Valencia, 2010). 
For instance, Schliemann and Carraher (2002) showed that Brazilian street children were able to solve math problems well with concrete objects (e.g., fruits) while selling goods on the market, but not in a formal test setting using paper-and-pencil assessments that have abstract contents (e.g., numbers). A recent study from Banerjee, Bhattacharjee, Chattopadhyay, \& Ganimian, (2017) also suggests that children who work on markets have arithmetic skills that are untapped by the school system. In this paper, we focus on unintended (mis-)matches.

\section{Language in math tests}

Certain features of math test items can affect performance when students are second language learners or have a low socioeconomic background, unrelated to their average math-performance. First, complexity of language applied in math tests affects students' performance (e.g., Abedi \& Lord, 2001). 'Word problems', where information on the problem is presented as text rather than in mathematical notation, are notorious for their disadvantageous effects on math performance for students from underprivileged backgrounds and second language learners (Carpenter, 1980; Haag et al., 2013). In addition, complexity on a descriptive level (e.g., text length), lexical level (i.e., academic word use), and grammatical level (e.g., number of complex sentences) cause performance disadvantages (Haag et al., 2013). In addition to general language features of items, mathematical language features (e.g., the use of quantitative language, such as 'many' and 'fewer', and spatial language, such as 'near' and 'above') also account for substantial variance in math performance (Purpura \& Reid, 2016; Ramani, Zippert, Schweitzer, \& Pan, 2014). Deficits in mathematical language understanding affect math performance (Purpura \& Reid, 2016).

Complexity of language in math tests is more disadvantageous for low-SES students than for high-SES students, probably, at least partly, because in low-SES home environments, children have on average less access to complex, academic, or mathematical language than children do in high-SES home-environments (Hart \& Risley, 1995; Purpura \& Reid, 2016; Magnuson, Sexton, Davis-Kean, \& Huston, 2009; Vandermaas-Peeler, Nelson, Bumpass, \& Sassine, 2009). As a consequence, complexity of language in math-items reinforces the SES-gap in academic performance.

\section{Context in math items}

In addition to the complexity of language, an important question is whether context applied in math-items may affect low-SES students' performance. In particular, math items may differ in the context they use to describe a mathematical problem. For instance, a question in which students are asked to divide 240 by 6 has no specific context, while a question that asks to distribute 240 euro among 6 friends has context related to money and social relations. 
Whereas effects of the context of items have not received attention in relation to SES, effects of context have been examined in research on gender differences in math performance. Some of these studies did not find a relation between gender specific item context and differences in performance between boys and girls (Chipman, Marshall, \& Scott, 1991; Sappington, Larsen, Martin, \& Murphy, 1991), but other studies found evidence that girls' performance in particular is impaired by stereotypically boys' context (Leder \& Forgasz, 2018; Zohar \& Gershikov, 2008), perhaps because of less familiarity with this context (cognitive hypothesis), and, as a result, less motivation to work with this type of context (affective hypothesis) (Zohar \& Gershikov, 2008). In this paper, we focus on context that might be specifically familiar for low-SES students and therefore may impact their performance.

\section{Context relevant in low-SES environments}

We reasoned that context that is related to recurrent problems in low-SES environments, might influence low-SES students' performance, because low-SES students may be more familiar with this context than with other contexts applied in math tests. In addition, we reasoned that high-SES students may be equally familiar with all type of contexts applied in math test items, because of typical features in their environments, such as parental language and parent-child play interactions, that match with educational contexts and math tests (Hart \& Risley, 1995; Purpura \& Reid, 2016; Magnuson et al., 2009; VandermaasPeeler et al., 2009). Therefore, we expected to find a positive relation between low-SES relevant context in math tests and performance among low-SES students, and not to find such a relation among high-SES students.

What context of test items may be related to recurrent problems for low-SES students? First, low-SES environments can be characterized by a higher chance on the presence of a variety of threats, such as physical dangers, threats to job stability, and resource scarcity, compared to high-SES environments (Chaby et al., 2016; Ellis \& Del Giudice, 2014; Kraus, Horberg, Goetz, \& Keltner, 2011; Kraus, Piff, Mendoza-Denton, Rheinschmidt, \& Keltner, 2012). Characteristic to low-SES is the risk for experiences of scarcity of money (APA task force Socioeconomic Status, 2007). In addition, food insecurity, a state in which reliable access to adequate food is limited by a lack of money and other resources, is also related to low-SES and poverty (Barrett, 2010). Although severe scarcity of food is a typical consequence of disasters such as war, drought, or earthquakes, in all countries, also the most developed ones, food insecurity occurs as a consequence of chronic poverty (Barrett, 2010).

Obviously, some of the treats related to low-SES are generally not included in math items, because they are inappropriate for youth in some way, such as items on physical dangers. However, some of these contexts may often be used, such as items related to scarce resources: specifically, food or money (e.g., an item on how to divide a pizza in 5 equal slices, or how to share a certain amount of money equally with 5 persons). On these grounds, we reasoned that context and problems related to resources (e.g., the availability 
of food and money) might be applied in math tests and at the same time be particularly relevant to low-SES students.

In addition, low-SES environments tend to be less predictable than high-SES environments, i.e., there is greater uncertainty about future environmental outcomes (Frankenhuis, Panchanathan, \& Nettle, 2016). For example, unpredictable influences like unsafety in their neighborhoods, crime, and threats to job stability, may limit low-SES individuals' opportunities to meet their basic life needs, such as safety and income. As a result, for low-SES individuals, external, social factors in the environment seem to be prioritized above internal, individual factors (Kraus et al., 2012). In addition, because of this environmental unpredictability, social safety nets are more important, inducing a stronger focus on external social factors, such as social relationships, hierarchy, and the thoughts and intentions of others among people from low-SES environments than among people from high-SES environments (Kraus et al., 2012; Varnum, Grossmann, Kitayama, \& Nisbett, 2010). More specifically, context and problems involving social interactions, like trading and negotiating, or social comparisons, like sports, may be related to recurrent problems in low-SES environments and may therefore be particularly familiar and relevant for students from low-SES environments.

Thus, topics that may have a different status for low compared to high-SES students include (1) scarce resources, i.e., money and food, and (2) social interactions, such as negotiating and sharing. There are a number of theoretical angles to predict how this content may influence performance.

\section{Relevant context may enhance math performance}

Generally speaking, literature on the contextual nature of cognition suggests the importance of environmental features in cognitive development (Bronfenbrenner, 1979; Ceci, 1990, 1993). For example, the theory of successful intelligence (Sternberg, 2005, 2014) suggests that intelligence is the ability to set and realize goals in life, given the cultural context. On this view, socioeconomic status can be considered as 'culture,' because it also refers to a shared context that requires specific adaptive competencies and practical knowledge (Kraus et al., 2012; Sternberg, 2014). Basic information processing skills underlying intelligence may be shared across cultures, but what differs between cultures is the nature of problems that people encounter in their environments. These environmental differences in recurrent problems lead to differences in development of practical knowledge and adaptive competences (Sternberg, 2014), which in turn may impact the capacities to adapt to and learn in formal school settings (Schliemann \& Carraher, 2002).

Based on the theory of successful intelligence, it can be expected that low-SES students will show enhanced performance in handling problems with these familiar topics compared to resolving neutral problems (e.g., abstract problems), because of practical knowledge and adapted cognitive capacities they have developed in interaction with these recurrent problems. In addition, because children in high-SES environments may experience less 
severe problems or dependencies regarding scarcity of resources and social relations, and because they may experience a broader range of challenges and problems in interaction with their parents, high-SES students may benefit less from these typical low-SES familiar context in math tests.

\section{Relevant context may diminish math performance}

However, literature on stereotype threat offers a contradictory prediction regarding the use of low-SES familiar context. Central to the theory about stereotype threat is the experience of uncertainty among members of stigmatized groups about whether those they interact with will judge them according to predominant stereotypes associated with their stigmatized identity (Murphy \& Taylor, 2012). Specifically, in school-settings, people are sensitive to stereotype threat (Steele \& Aronsson, 1995), and research has consistently shown that - often subtle - cues that remind people of their stigmatized group identity can affect school performance (Murphy, Steele, \& Gross, 2007; Murphy \& Taylor, 2012; Nguyen \& Ryan, 2008; Spencer, Logel, \& Davies, 2016; Walton \& Spencer, 2009). Because lowSES students' risk of confirming a negative reputation of low intellectual ability (Croizet, \& Claire, 1998), low-SES familiar context in math items might function as a cue to remind students on negative stereotypes related to their stigmatized group identity, which may diminish their performance.

In addition, theories on attention processes also predict disadvantage for low-SES students regarding the use of low-SES familiar context, especially regarding context like money and food. Because these topics may be highly rewarding for low-SES students, they may distract them from their current task (Anderson, Laurent, \& Yantis, 2011). In addition, scarcity may narrow people's 'mental bandwidth', taking a toll on people's attention, cognition, and self-control. In particular, reduced bandwidth impairs executive control, diminishing people's ability to plan and increasing impulsiveness (Mullainathan \& Sharif, 2013). So, when resources like money or food are included in complex math-items, the association with scarcity may change low-SES (and not high-SES) students' cognitive state, harming their performance on the current test.

\section{Current research}

We examine these contradictory possible effects of SES relevant context on performance. Because to date little is known about possible effects of context on performance in the relation to SES, we first conducted a pilot study (Study 1) to assess whether math-items with context that is especially familiar for low-SES students (low-SES familiar context) show an effect to the advantage of low-SES students. We examined 20 items with lowSES familiar context, and 20 items with neutral context. Results of this pilot study show that nine out of 20 items with low-SES familiar context show a significant bias to the disadvantage of low-SES students, and we did not find this pattern among the neutral items. A replication of this procedure (testing 20 items with low-SES familiar context) showed the same pattern of results. However, the small selections of items did not allow 
us to test whether the difference between items with and without low-SES familiar context was statistically significant, nor to control for relevant features from items and students.

Therefore, in Study 2, we tested with a substantially larger number of items ( $N=161$ items) whether items with low-SES familiar context indeed show bias to the disadvantage of low-SES students significantly more often than other items, controlling for features of items that are known to affect math-performance for low-SES students or second language learners. Third, in Study 3, in order to evaluate whether the relations we find are generalizable over countries, we additionally evaluated the same data on student-level ( $N=1,018,330$ students). We tested whether there is a significant interaction effect between students' SES and low-SES familiar context in test items on math-test performance to the disadvantage of low-SES students, controlling for relevant features items and students' countries.

Because of the world-wide use of math tests, in all stages of students' educational pathways before entrance to tertiary education, and the important role of performance on math tests in regarding certification and admission decisions, we test our research questions with international data, containing results from students in 58 countries, both in primary and secondary education.

\subsection{Study 1: pilot study}

Before starting our preliminary analyses, we hypothesized that low-SES familiar context would enhance low-SES students' performance on math tests. Therefore, we explored in a small set of randomly selected math-items ( $N=20$ items) whether items with lowSES familiar context showed bias in favor of low-SES students, and whether this bias was absent in items ( $\mathrm{N}=20$ items) with neutral context (both items with word problems, and items with only mathematical notation).

\section{Method}

In line with current recommendations about research practices, (Wagenmakers, Wetzels, Borsboom, van der Maas, \& Kievit, 2012) we preregistered the data source, definitions, and statistical plan for Differential Item Functioning (DIF) analyses of our study at the Open Science Framework (see https://osf.io/geqkp/). In all studies (Study 1, 2, and 3), we applied the data source, definitions and plan for DIF-analyses from this preregistration. In Study 2 and Study 3, we added exploratory analyses that were not preregistered.

We used the data from Trends in International Mathematics and Science Studies (TIMSS ${ }^{2}$ ). We used released items from cohort 2007 and $2011(N=1,018,330)$ from all participating

${ }^{2}$ TIMSS. Copyright (C) 2009 International Association for the Evaluation of Educational Achievement (IEA). Publisher: TIMSS \& PIRLS International Study Center, Lynch School of Education, Boston College. 
countries ( 57 in 2007, 58 in 2011). TIMSS defines its international target populations in terms of the amount of years of schooling students have received. The international target populations for TIMSS are 1) students in their fourth year of formal schooling, and 2) students in their eighth year of formal schooling. Because we had no specific hypothesis about years of formal schooling or the age when SES-background may bias math test outcomes, we included students from both of the available grades: grades 4 (average age 9.5 years), and 8 (average age 13.5 years).

SES. Finding comparable indicators for social background in international educational studies is difficult, for example, because social background is defined differently across countries, and students may not know details about their parents' educational level, occupational status, and income (e.g., Torney-Purta, Lehmann, Oswald, \& Schulz, 2001). Initially, our plan was to apply two proxies for SES, as recommended by the APA task force Socioeconomic Status (2007). As a first proxy for SES, we planned to apply a measure based on parental educational level, based on participants' rating of the educational level of their mother and father on the international ISCED-classification on a 6-point scale ( $1=$ no education, 6 = university degree). As second proxy for SES, we planned to apply a measure based on the number of books available at the students' home, a widely used SES proxy in cross-national research (Brunello, Weber, \& Weiss, 2017). Number of books captures access to cultural capital, an aspect of SES associated with educational achievement (Yang \& Gustafsson, 2004). Participants were asked to give an estimation of the number of books in their home ("About how many books are there in your home? Do not count magazines, newspapers, or your school books."). Participants indicated their estimation by choosing one out of five categories: 0-10 books; 11-25 books; 26-100 books; 101-200 books, and more than 200 books. A higher score indicates a higher SES on a scale from 1-5 (low-high).

When analyzing descriptive statistics of our first proxy for SES, parents' educational level, it appeared that 20 to 25 percent of the participants indicated that they did not know their parental educational level. In addition, the validity of the answers of the other participants may not be beyond question (Torney-Purta et al., 2001). Therefore, we decided to apply the number of books in home as primary indicator of socioeconomic status, before conducting any hypothesis tests. This decision is in line with previous international studies that have proven that number of books in home is a consistent proxy for social background, related to resources available for education, home literacy, and academic support in families (e.g., Beaton, 1996).

Classification of items. In Study 1, we randomly selected 20 items with low-SES familiar context (see Table A1), and 20 items with neutral context (see Table A3) for analyses of math tests applied by TIMSS in 2007 and 2011 in grade 4 and grade 8 . We define items with 'low-SES familiar context and problems' as items with mathematical problems involving 1) food 2) money) or 3) social interaction (e.g., competition, working together). We define items with 'low-SES neutral context and problems' as items with mathematical problems involving 1) word problems with neutral content (e.g., buttons, frogs) or 2) mathematical notation (e.g., $5631+286=$...). First, one researcher coded items according to these 
definitions. Second, a researcher who was not involved in this study rated these items on the same categories. The overlap between the first researcher's rating and the second researcher's rating was $82 \%$, which is higher than the commonly recommended $80 \%$ agreement as the minimum acceptable interrater agreement (McHugh, 2012). The conflicts of judgments were evaluated until full agreement was reached.

DIF-analyses. In order to detect if low-SES students are more likely to give the correct answer when items contain low-SES familiar context or problems giving their true math ability, we conducted Differential Item Functioning (DIF) analyses for SES-background. Items only show DIF if students from different backgrounds with the overall performance on the test have a different probability of giving the correct response on this specific question. Therefore, in order to detect DIF, we needed a measure for average math performance as matching criterion. TIMSS distinguishes between three cognitive domains - Knowing, Applying, and Reasoning - which represent different levels of math development and independently predict future math development (Olson, Martin, \& Mullis, 2008). For each domain, TIMSS estimates students' ability by estimating their 'Plausible Value'. In study 1 , we considered these Plausible Values as a matching criterion for the DIF analyses (Olson et al., 2008), applying for each item the Plausible Value related to the item's cognitive domain.

In this pilot study, we analyzed for each item separately whether there was DIF for SESbackground, and if so, whether the DIF was in favor of the low-SES students or the high-SES students. We conducted DIF analyses with Mantel-Haenszel (MH) procedure. A statistically significant chi-square identifies DIF, resulting from comparing item performance in the low-SES groups with the high-SES group after matching on Plausible Values. In addition, because applying more than one analyses to detect DIF is recommended in order to reduce the risk of Type I error, we decided to use Logistic Regression analyses (LR) as an additional method to detect DIF (Rogers \& Swaminathan, 1993; Swaminathan \& Rogers, 1990). In order to defect uniform DIF, we applied LR with item response as dependent variable, and Plausible Values, and SES-background as independent variables (Stark, Chernyshenko, \& Drasgow, 2006).

\section{Results}

First, we conducted DIF-analyses with the 20 randomly selected items containing lowSES familiar context. We applied MH and LR for each item, and only when both analyses for one item detected significant DIF, we concluded that the item showed DIF. With this conservative criterion, results showed that nine out of the 20 selected items with low-SES familiar context showed DIF to the disadvantage of low-SES students (45\%) (see Table $\mathrm{A} 2$ ). LR showed that these effects within these nine items are large, indicating $12 \%$ (on the item showing least DIF) to $42 \%$ (on the item showing most DIF) lower chance for lowSES students to give the correct answer on an item, compared to high-SES students with the same math-ability. In addition, 11 items (55\%) did not show DIF in relation to SESbackground. 
Next, we analyzed for each randomly selected neutral item (see Table A3) separately whether there was DIF for SES-background, applying the same definitions and methodology as in our primary analyses. For the neutral items with mathematical notation, seven out of 10 items (70\%) did not show DIF, two out of 10 items (20\%) showed DIF to the disadvantage of low-SES students, and one item (10\%) showed DIF in favor of low-SES students. For items with neutral items with word problems, eight items did not show DIF (80\%), and two items (20\%) showed DIF to the disadvantage of low-SES students (see Table A4).

In order to check the robustness of our unexpected findings regarding low-SES familiar items, we replicated our preliminary study by conducting the same primary analyses as in our preregistered research plan, with data from earlier waves of TIMSS (1999 and 2003 instead of 2007 and 2011, see Table A5). We used the same procedures and definitions as in our first analyses with these new datasets. Results showed the same pattern as in our first analyses: of 20 items with low-SES familiar context, eight showed DIF (40\%) to the disadvantage of low-SES students (see Table A6).

\section{Discussion}

Results of our pilot analyses in Study 1, both from first analyses and a replication, indicate that items with low-SES familiar context do not enhance math-performance among lowSES students, but on the contrary, that these items seem to diminish their performance. In addition, results suggest that items with neutral context do not bias low-SES students' performance. However, because we preliminary analyzed DIF for a small set of randomly selected items, these data do not allow us to tests whether this bias occurs statistically more often in items with low-SES familiar context compared to items with neutral context. In addition, this small sample of selected items does not allow us to test the role of other features of items known to affect low-SES students' math performance, such as linguistic complexity and academic word use, in addition to their context.

\subsection{Study 2}

In contrast to Study 1 , where we tested randomly selected items separately on the existence and extend of bias related to SES background, in Study 2, we analyzed with two complete tests from TIMSS 2003 and 2007 on item level $(\mathrm{N}=161)$ whether DIF to the disadvantage of low-SES students occurs statistically more in items with low-SES familiar context, controlling for other relevant features of items, such as linguistic complexity.

\section{Method}

In Study 2, methods are identical to those of Study 1 regarding data source, definitions, and DIF-analyses (MH). One difference between the approach in Study 1 and Study 2, however, concerns the matching criterion used in the DIF analyses. In contrast to Study 1 , were we used Plausible Values as matching criterion, in Study 2, we used the percentage 
of released items correctly answered on the math test as overall test score, without distinguishing between cognitive domains. We were able to apply the overall test score in study 2 as matching criterium because we included all released items for each of the two tests.

Data. We conducted DIF-analyses for all 161 items (Grade 4 and Grade 8, 2007 and 2011) using $\mathrm{MH}$ with students' individual score as matching criterion. We used a dichotomous measure for SES ( $1=$ low, o = high) because $\mathrm{MH}$ does not allow scale variables. This procedure resulted in information for all 161 items about the occurrence of DIF to the disadvantage of low-SES ( $1=$ yes, $0=$ no), and odds (measure for the amount and direction of DIF) and allowed us to conduct analyses at the level of items.

Linguistic features of items. In line with Haag et al. (2013), we coded all items concerning linguistic features on several levels. Regarding descriptive features, we counted for each item total words, number of different words, total number of characters, number of characters without spaces, average syllables per word, number of sentences, and averages sentence length in words, applying an online tool provided by Textalyser (http://textalyser. net/). In addition, we coded all items regarding the use of academic words ( $1=$ at least one academic word, $\mathrm{o}=$ no academic words), applying the Academic Word List (Coxhead, 2000).

Furthermore, two general areas of mathematical language are frequently distinguished in literature. First, quantitative language (such as 'many', 'fewer', 'less', and 'more') is related to comparisons between groups and numbers (e.g., Barner, Chow, \& Yang, 2009). Second, spatial language (such as 'near', 'above', and 'before'), refers to relations between objects and numbers on a line (e.g., Ramani et al., 2014). We coded all items with regard to the use of quantitative language $(1=$ at least once, $0=$ no), and spatial language $(1=$ at least once, $\mathrm{o}=\mathrm{no})$.

\section{Results}

Table 1 shows descriptive statistics of the items. Table 2 shows differences between items with and without low-SES familiar context on several features. We compared items with low-SES familiar context with other items with neutral context (word-problems, and items with only mathematical notation).

Table 2 shows that math items containing low-SES familiar context show significantly more DIF to the disadvantage of low-SES students than items containing word problems with other context and items with only mathematical notation. From items with low-SES familiar context, $51 \%$ showed DIF to the disadvantage of low-SES, compared to $26 \%$ and $6 \%$ in the other item-categories. In addition, the odds (DIF analyses, MH) for low-SES students to give the correct answer, given their average math performance, is significantly lower (0.91) in math items with low-SES familiar context than in the other items (1.02 and 1.06). 
TABLE 1 Descriptive Statistics of 161 Math Items from TIMSS 2003 and 2007

\begin{tabular}{|c|c|c|c|c|}
\hline & $\begin{array}{l}\text { Proportion } \\
\text { or mean }\end{array}$ & SE & Min & Max \\
\hline \multicolumn{5}{|l|}{ Grade } \\
\hline Grade 8 & 0.55 & 0.04 & 0 & 1 \\
\hline Grade 4 & 0.45 & 0.04 & 0 & 1 \\
\hline \multicolumn{5}{|l|}{ Item-type } \\
\hline Multiple Choice & 0.53 & 0.04 & 0 & 1 \\
\hline Constructed response & 0.47 & 0.04 & 0 & 1 \\
\hline \multicolumn{5}{|l|}{ Context domain } \\
\hline Algebra & 0.11 & 0.02 & 0 & 1 \\
\hline Data and Chance & 0.11 & 0.02 & 0 & 1 \\
\hline Data Display & 0.06 & 0.02 & 0 & 1 \\
\hline Geometric Shapes and Measures & 0.15 & 0.03 & 0 & 1 \\
\hline Geometry & 0.14 & 0.03 & 0 & 1 \\
\hline Number & 0.44 & 0.04 & 0 & 1 \\
\hline \multicolumn{5}{|l|}{ Cognitive domain } \\
\hline Knowing & 0.35 & 0.04 & 0 & 1 \\
\hline Applying & 0.46 & 0.04 & 0 & 1 \\
\hline Reasoning & 0.19 & 0.03 & 0 & 1 \\
\hline \multicolumn{5}{|l|}{ Type of problem } \\
\hline Word problem & 0.90 & 0.02 & 0 & 1 \\
\hline Mathematical notation & 0.10 & 0.02 & 0 & 1 \\
\hline \multicolumn{5}{|l|}{ General language features } \\
\hline Total word count & 15.88 & 0.88 & 0 & 57 \\
\hline Number of different words & 12.31 & 0.60 & 0 & 40 \\
\hline Total number of characters & 153.61 & 8.60 & 7 & 579 \\
\hline Number of characters without spaces & 83.14 & 4.82 & 0 & 347 \\
\hline Average Syllables per Word & 1.47 & 0.02 & 0 & 2 \\
\hline Sentence count & 3.66 & 0.17 & 1 & 12 \\
\hline Averages sentence length in words & 9.69 & 0.34 & 0 & 26 \\
\hline Academic word use (AWL) & 0.34 & 0.04 & 0 & 1 \\
\hline \multicolumn{5}{|l|}{ Math language features } \\
\hline Quantitative language & 0.55 & 0.04 & 0 & 1 \\
\hline Spatial language & 0.45 & 0.04 & 0 & 1 \\
\hline \multicolumn{5}{|l|}{ Low-SES familiar context } \\
\hline General (all low-SES familiar context) & 0.32 & 0.04 & 0 & 1 \\
\hline Money and trading & 0.13 & 0.03 & 0 & 1 \\
\hline Food & 0.04 & 0.02 & 0 & 1 \\
\hline Social interaction & 0.17 & 0.03 & 0 & 1 \\
\hline \multicolumn{5}{|l|}{ Differential Item functioning (DIF) } \\
\hline DIF to the disadvantage of low-SES students $(\mathrm{MH})$ & 0.32 & 0.01 & 0 & 1 \\
\hline Odds (MH) & 0.99 & 0.02 & 0.57 & 1.56 \\
\hline
\end{tabular}

Note. $N=161$ items 
TABLE 2 Differences between Items with Low-SES familiar context, Items with Neutral Context (Word Problems and Items with only Mathematical Notation)

\begin{tabular}{|c|c|c|c|}
\hline & \multirow[t]{2}{*}{ Low-SES familiar context } & \multicolumn{2}{|c|}{ Neutral context } \\
\hline & & Word problems & $\begin{array}{l}\text { Mathemati- } \\
\text { cal notation }\end{array}$ \\
\hline \multicolumn{4}{|l|}{ Grade } \\
\hline Grade 8 & 0.57 & 0.50 & 0.75 \\
\hline Grade 4 & 0.43 & 0.50 & 0.25 \\
\hline \multicolumn{4}{|l|}{ Item-type } \\
\hline Multiple Choice & 0.33 & $0.57^{\star \star}$ & $0.94^{\star \star}$ \\
\hline Constructed response & 0.69 & $0.43^{* \star}$ & 0.06 \\
\hline \multicolumn{4}{|l|}{ Context domain } \\
\hline Algebra & 0.04 & 0.09 & $0.44^{*}$ \\
\hline Data and Chance & 0.22 & 0.06 & 0.00 \\
\hline Data Display & 0.14 & $0.01^{\star \star}$ & 0.06 \\
\hline Geometric Shapes and Measures & 0.04 & $0.21^{\star \star}$ & 0.13 \\
\hline Geometry & 0.02 & $0.21^{\star \star}$ & 0.06 \\
\hline Number & 0.55 & 0.41 & 0.31 \\
\hline \multicolumn{4}{|l|}{ Cognitive domain } \\
\hline Knowing & 0.24 & 0.36 & $0.69^{*}$ \\
\hline Applying & 0.45 & 0.51 & $0.19^{*}$ \\
\hline Reasoning & 0.31 & $0.13^{\star \star}$ & 0.13 \\
\hline \multicolumn{4}{|l|}{ General language features } \\
\hline Total word count & 20.71 & $15.12^{\star \star}$ & $4.94^{\star \star \star}$ \\
\hline Number of different words & 15.61 & $11.87^{\star \star}$ & $4 \cdot 38^{\star \star \star}$ \\
\hline Total number of characters & 202.12 & $144 \cdot 48^{\star \star}$ & $52.69^{\star \star \star}$ \\
\hline Number of characters without spaces & 110.65 & $78.63^{\star \star}$ & $22.00^{\star \star \star}$ \\
\hline Average Syllables per Word & 1.50 & 1.50 & $1.25^{\star \star}$ \\
\hline Sentence count & 4.45 & $3.49^{*}$ & $2.19^{\star \star}$ \\
\hline Averages sentence length in words & 10.86 & 9.91 & $4.67^{\star \star \star}$ \\
\hline Academic word use (AWL) & 0.39 & 0.34 & 0.19 \\
\hline \multicolumn{4}{|l|}{ Math language features } \\
\hline Quantitative language & 0.76 & $0.51^{\star \star}$ & $0.06^{\star *}$ \\
\hline Spatial language & 0.47 & 0.47 & 0.25 \\
\hline \multicolumn{4}{|l|}{ Differential Item functioning (DIF) } \\
\hline DIF to the disadvantage of low-SES students (MH) & 0.51 & $0.26^{\star \star}$ & $0.06^{*}$ \\
\hline Odds (MH) & 0.91 & $1.02^{\star \star \star}$ & $1.06^{*}$ \\
\hline
\end{tabular}

Note. All significance tests compared to items with low-SES familiar context (first column). Bold numbers indicate a significant difference ${ }^{\star} P<0.05,{ }^{\star \star} P<0.01,{ }^{\star \star \star} p<0.001, N=161$ items

To test whether these differences remain significant after controlling for other important features of items that can affect low-SES students' performance, we conducted linear regression analysis with DIF-odds as dependent variable, low-SES familiarity as predictor, and all relevant variables (bold) in Table 2 as covariates. Results show that for items with low-SES familiar context, low-SES students' odds to report the correct answer is lower 
than high-SES students' odds, compared to their overall performance on the test and other features of items (MH: $b=-0.09, t(160)=-2.55, p=0.012)$.

In addition, we analyzed DIF-odds separately for our relevance categories: money, food and social interaction. Results suggest that especially items containing context that refers to money and social interaction are related to significant less chance of reporting the correct answer among low-SES students compared to high-SES students with the same math ability (Figure 1). Context related to food did not show a significant difference with word problems with neutral context.

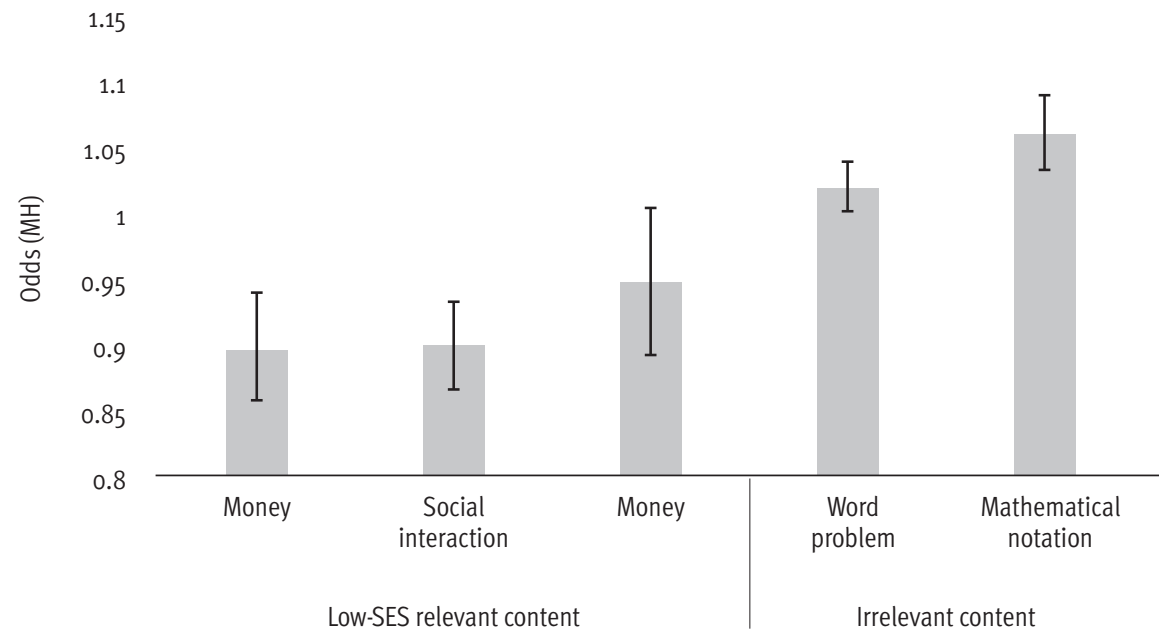

FIGURE 1 Odds resulting from DIF-analysis on SES background and context of math-items, showing that especially low-SES familiar context 'money' and 'social interaction', compared to neutral context (word problems and mathematical notation), leads to less chance of reporting the correct answer among low-SES students, compared to high-SES students with the same math ability $(\mathrm{N}=162)$.

\section{Discussion}

We find evidence that math items containing word problems with low-SES familiar are related to less chance of reporting the correct answer for low-SES students, compared to word problems without relevant context. Moreover, within items with low-SES familiar context, items referring to 'money' and 'social interaction' carry these findings. We did not find evidence that items containing context related to food predict bias to the disadvantage of low-SES students. 


\subsection{Study 3}

The results of Study 2 suggested that low-SES familiar context may work against low-SES students' performance. In study 3, we aimed to test whether these relations found in Study 2 are present in all countries, or whether these relations can be explained by differences between countries. Therefore, in Study 3, we analyzed the data on student level, using the nested structure (students within countries) and repeated measures structure (students reported answers to several items) of our data. This enabled us to test whether there is an interaction between low-SES familiar context and low-SES status on students' performance on student-level, controlling for students' country.

\section{Method}

In Study 3, methods are identical to those of in Study 1 regarding data source and definitions. Regarding the DIF-analyses, the procedure using students' average test score as matching criterion was identical to Study 2.

Analyses. We conducted mixed logistic regressions analyses with performance on an item (1 = correct answer, $\mathrm{O}=$ incorrect answer) as a dependent variable, students SES-background (scale 1-5; 1 = low, 5 = high) with familiarity category (low-SES familiar vs. low-SES neutral) as an interaction term, students SES-background (scale 1-5; $1=$ low, $5=$ high) and average individual test score as between subjects factors, familiarity category (low-SES familiar vs. low-SES neutral) as a within subjects factor, and features of items (word problem, item type, context domain, cognitive domain, total word count, number of different words, total number of characters, number of characters without spaces, average syllables per word, sentence count, average sentence length, academic words, quantitative language, spatial language, and country-dummies) as covariates. We conducted these analyses separately for grades 4 and 8.

\section{Results}

Table 3 show descriptive statistics from our samples in Study 3.

Analyses show both in grade 4 (Table 4, Table A7) and grade 8 (Table 4, Table A8) a significant interaction between SES-background and low-SES familiar context, indicating that low-SES students have a 16\% (grade 8, Exp $(B)=0.84$ ) to 18\% (grade 4, Exp (B) = 0.82) lower chance of reporting the correct answer to items with low-SES familiar context than students from the highest SES-background, given their average math performance (See also Figure 2).

In addition, we have conducted the same analyses as reported in Table A7 and Table A8, but separately for low-SES familiar context money, food, and social interaction. Results for the interaction-terms are shown in Table 4. These results indicate that the interaction we found depends on context related to money, and social interaction. 
TABLE 3 Descriptive Statistics from Samples in Study 3 regarding SES and Country (Grade 4)

\begin{tabular}{|c|c|c|c|c|c|}
\hline \multicolumn{6}{|l|}{ SES (1 = low. $5=$ high), $\%$} \\
\hline SES $_{1}$ & 18.0 & & & & \\
\hline SES 2 & 24.7 & & & & \\
\hline $\mathrm{SES}_{3}$ & 29.5 & & & & \\
\hline $\mathrm{SES}_{4}$ & 13.4 & & & & \\
\hline SES $_{5}$ & 11.0 & & & & \\
\hline \multicolumn{6}{|l|}{ Country, \% } \\
\hline Algeria & 2.2 & Israel & 1.3 & Slovenia & 1.6 \\
\hline Australia & 1.7 & Italy & 1.8 & Sweden & 2.1 \\
\hline Bahrain & 1.7 & Japan & 1.8 & Syria. Arab Republic of & 1.9 \\
\hline Armenia & 1.9 & Jordan & 2.1 & Thailand & 2.2 \\
\hline Bosnia and Herzegovina & 1.7 & Korea. Republic of & 1.7 & Tunisia & 1.7 \\
\hline Botswana & 1.7 & Kuwait & 1.7 & Turkey & 1.8 \\
\hline Bulgaria & 1.6 & Lebanon & 1.5 & Ukraine & 1.8 \\
\hline Chinese Taipei & 1.6 & Lithuania & 1.6 & Egypt & 2.7 \\
\hline Colombia & 2.0 & Malaysia & 1.8 & United States & 3 \\
\hline Cyprus & 1.8 & Malta & 1.9 & Serbia & 1.6 \\
\hline Czech Republic & 2 & Mongolia & 1.8 & England & 1.6 \\
\hline El Salvador & 1.7 & Morocco & 1.2 & Scotland & 1.7 \\
\hline Georgia & 1.7 & Oman & 1.9 & Spain (Basque Country) & 0.9 \\
\hline Palestinian National Authority & 1.8 & Norway & 1.9 & United Arab Emirates (Dubai) & 1.3 \\
\hline Ghana & 2.2 & Qatar & 2.9 & Canada (Ontario) & 1.4 \\
\hline Hong Kong. SAR & 1.4 & Romania & 1.7 & Canada (Quebec) & 1.6 \\
\hline Hungary & 1.7 & Russian Federation & 1.8 & Canada (British Columbia) & 1.7 \\
\hline Indonesia & 1.7 & Saudi Arabia & 1.7 & United States (Massachusetts) & 0.8 \\
\hline Iran. Islamic Republic of & 1.6 & Singapore & 1.9 & United States (Minnesota) & 0.7 \\
\hline
\end{tabular}

Note. $N=2,779,383$

\section{Discussion}

We find a significant interaction between low-SES familiar context in math-items and the chance of giving the correct answer to the disadvantage of low-SES students, given students' math ability. These effects are particularly related to the use of context related to money and social interaction. Controlling for country, language difficulty, cognitive domain, and content domain of items does not change the results.

\subsection{General discussion}

Overall, our findings suggest that context in math test items related to money and social interactions hinders low-SES students' performance. Low-SES students perform less well than expected based on their average performance on items containing this 'familiar' context than on items containing neutral context (items with word problems or 
items with only mathematical notation). The effects are substantial: students from low-SES backgrounds have on average $16 \%$ (grade 8 ) to $18 \%$ (grade 4 ) lower chance of giving the correct answer when items contain this relevant context, given students' average test score (i.e., their average test score). These effects cannot be explained by linguistic complexity, nor by differences in content domain or cognitive domain between items.

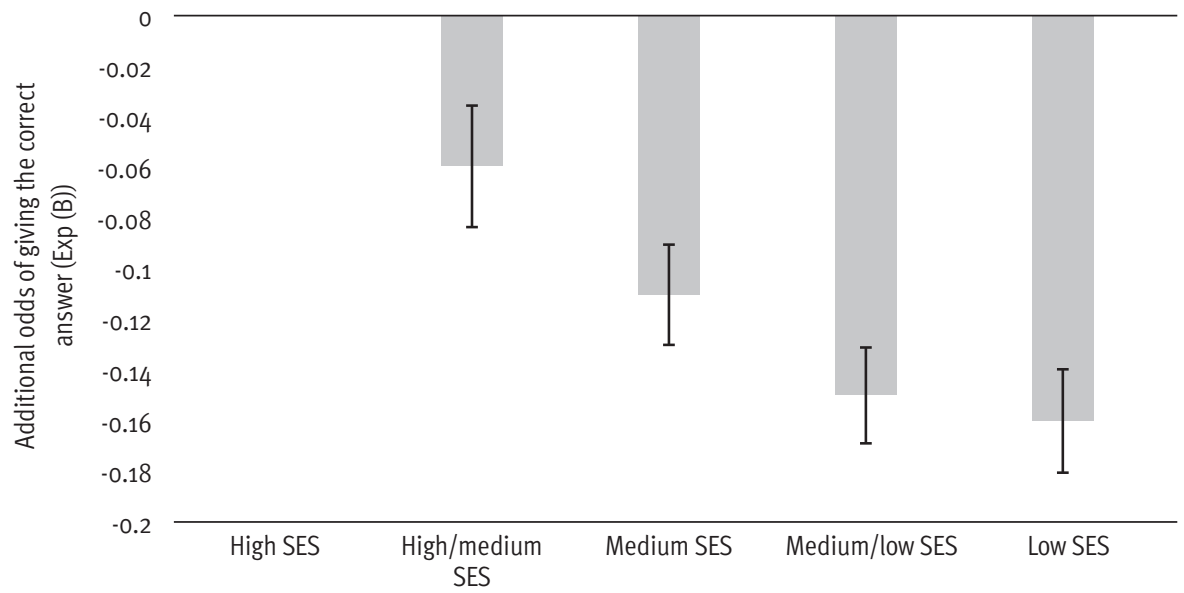

FIGURE 2 Interaction between SES-background and low-SES familiar context: the decrease of students' odds to report the correct answer compared to high-SES students, controlling for students' math ability, word problem, item type, context domain (3 dummy-variables), cognitive domain ( 2 dummy-variables), total word count, number of different words, total number of characters, number of characters without spaces, average syllables per word, sentence count, average sentence length, academic words, quantitative language, spatial language, and country ( 58 dummy-variables) (grade 8 , results from grades 4 and 8 show the same pattern).

TABLE 4 Results from Logistic Repeated Measures Analyses Predicting Change of Giving the Correct Answer among Students in Grade 4 and Grade 8 from Different SESBackgrounds by Relevance of Context of Items, Separate Analyses for Money, Food, and Social Interaction

\begin{tabular}{lllll}
\hline \multicolumn{4}{c}{ Grade 4} & \multicolumn{3}{l}{ Grade 8 } \\
\hline & Exp (B) & $95 \% \mathrm{Cl}$ & & $95 \% \mathrm{Cl}$ \\
\hline Low-SES familiar context (all) $\times$ SES & $0.82^{*}$ & {$[0.80,0.85]$} & $0.84^{*}$ & {$[0.84,0.88]$} \\
Money $\times$ SES & $0.82^{*}$ & {$[0.79,0.86]$} & $0.85^{*}$ & {$[0.85,0.90]$} \\
Food $\times$ SES & $0.78^{*}$ & {$[0.75,0.82]$} & 1.15 & {$[1.04,1.28]$} \\
Social interaction $\times$ SES & $0.88^{*}$ & {$[0.85,0.92]$} & $0.85^{*}$ & {$[0.82,0.87]$} \\
$N$ & 2779383 & & 2721782 & \\
\hline
\end{tabular}

Note. Interaction between lowest SES (1) and highest SES (5) and low-SES familiar context. In all models, the following covariates were included: students' math ability, word problem, item type, context domain (3 dummy-variables), cognitive domain (2 dummy-variables), total word count, number of different words, total number of characters, number of characters without spaces, average syllables per word, sentence count, average sentence length, academic words, quantitative language, spatial language, and country (57 dummy-variables in Grade 4, 58 dummy-variables in Grade 8), $\mathrm{Cl}=$ confidence interval, ${ }^{*} p<.001$. 


\section{Narrowed attention and cognitive control}

From a contextual perspective on development (Bronfenbrenner, 1979; Ceci, 1990, 1993; Ellis et al., 2017; Frankenhuis \& de Weerth, 2013; Sternberg, 2014), students would learn and perform better when context and problems in standardized tests match with their practical knowledge and adaptive competences. For low-SES students, in our current school-systems, there might me more often a mismatch between skills and knowledge learned in their home-environment and demands in school-settings than for highSES students (Ellis et. al., 2017). Therefore, it is important to examine whether context and problems that are more ecologically familiar for low-SES students may ameliorate their performance on, for instance, math tests. We initially expected that problems with resources like money, food, and social interaction, are particularly familiar for low-SES students, and therefore, could enhance their performance. However, our results show an opposite effect: content that is familiar for low-SES students, especially money and social interaction, works to the disadvantage of their math performance.

\section{Testing conditions}

Earlier research on school performance in low-SES students suggested that testing conditions are also an important factor in explaining students' performance (Carraher, Carraher, \& Schliemann, 1987; Nunes, Schliemann, \& Carraher, 1993). For example, Schliemann and Carraher (2002) showed that students, experienced with money transactions as street vendors, who were able to resolve mathematical exercises orally, performed worse on the same mathematical exercises when they used written algorithms. This worse performance seems to be a result of troubles in adopting written symbolic systems and procedures (Schliemann \& Carraher, 2002). In our study, our testing results are limited to testing conditions were students have to use written symbols and procedures to resolve mathematical problems. Therefore, the advantage of the relevance of context of items (e.g., money) may be limited by testing conditions that are not ecologically familiar (applying written symbols and algorithms). However, because testing conditions were the same for all items, the disadvantage of testing conditions cannot explain why low-SES students perform especially worse on items with low-SES familiar context.

\section{Attentional processes}

Literature on attentional processes suggests that highly valuable context in the face of scarcity may distract students from their task, and narrow attention and cognitive control, which may affect their performance on math (Anderson et al., 2011; Gable \& HarmonJones, 2008; Mullainathan \& Sharif, 2013). From this perspective, especially valued resources such as money and food would affect attentional processes. Although we found disadvantageous effects from context related to money, results did not suggest such an effect from context related to food. On the contrary, we found bias related to social interaction, which we would not expect from this attentional processes-perspective. 


\section{Stereotype threat}

Theory on stereotype threat predicts a diminished performance when students from a marginalized group receive cues that remind them of their stigmatized group identity (Murphy \& Taylor, 2012). Context in math-items that is especially relevant in low-SES environments, might remind low-SES people on their minority status, leading to less performance on math tests. Research has shown that cues that evoke stereotype threat are often subtle, and undermine students' performance (Murphy et al., 2007; Murphy \& Taylor, 2012; Nguyen \& Ryan, 2008; Walton \& Spencer, 2009). Our finding that content related to money and social interaction predicts diminished performance among low-SES students, regardless of their true math-ability, may be explained by stereotype threat effects. Future experimental research, and, for example testing the attenuating effects of self-affirmation interventions (Borman, Grigg, Rozek, Hanselman, \& Dewey, 2018) on the relations we found, is needed to understand whether stereotype threat is the core explanation of our finding.

\section{Limitations}

Our data are observational. Therefore, we cannot exclude the possibility that items with and without low-SES familiar context differ on relevant, unobserved features. Although we controlled for important features that are known to affect low-SES students' performance, it is possible that unknown features may still impact our results. In an experimental setting, it would be possible to test the effects of ecologically familiar context on math performance more systematically, by manipulating the context of items.

We applied students average test score, including items with all contexts, as their true math ability. Therefore, the biasing effects from relevant context probably plays a role in this measure. More specifically, low-SES students' true math ability may be underestimated. Therefore, the disadvantage from low-SES relevant contexts on math performance we report may be underestimated as well.

More research is needed to understand the extend and underlying mechanisms of our findings. For example, it is important to investigate which context, in addition to money and social interaction, may take along bias for certain SES-groups, and which context is 'neutral' for all. In addition, future research should investigate whether our findings generalize to testing in other educational domains, such as science or language. More insight in hidden bias in educational tests promotes equal opportunities for students from all socioeconomic background.

\section{Conclusion}

Our study offers an important start for further investigation of sources for social inequality in education by showing that context of math items may contribute to unintended biases 
in math tests for students from low-SES backgrounds. Because context such as money and social interaction seems to work against the performance of low-SES students in math test, test-developers and researchers should be cautious in using these topics in math tests. 


\subsection{References}

Abedi, J., \& Lord, C. (2001). The language factor in mathematics tests. Applied Measurement in Education, 14(3), 219-234.

American Psychological Association, Task Force on Socioeconomic Status. (2007). Report of the APA Task Force on Socioeconomic Status. Washington, DC.

Anderson, B. A., Laurent, P. A., \& Yantis, S. (2011). Value-driven attentional capture. Proceedings of the National Academy of Sciences, 108(25), 10367-10371.

Banerjee, P. A. (2016). A systematic review of factors linked to poor academic performance of disadvantaged students in science and maths in schools. Cogent Education, 3(1), 1-17.

Banerjee, A. V., Bhattacharjee, S., Chattopadhyay, R., \& Ganimian, A. J. (2017). The Untapped Math Skills of Working Children in India: Evidence, Possible Explanations, and Implications.

Barner, D., Chow, K., \& Yang, S. J. (2009). Finding one's meaning: A test of the relation between quantifiers and integers in language development. Cognitive psychology, 58(2), 195-219.

Barrett, C. B. (2010). Measuring food insecurity. Science, 327(5967), 825-828.

Beaton, A. E. (1996). Mathematics Achievement in the Middle School Years. IEA's Third International Mathematics and Science Study (TIMSS). Chestnut Hill, MA: TIMSS \& PIRLS International Study Center, Boston College.

Borman, G. D., Grigg, J., Rozek, C. S., Hanselman, P., \& Dewey, N. A. (2018). Self-affirmation effects are produced by school context, student engagement with the intervention, and time: Lessons from a district-wide implementation. Psychological science, 29(11), 17731784 .

Bronfenbrenner, U. (1979). The ecology of human development. Cambridge, MA: Harvard Univ. Press.

Brunello, G., Weber, G., \& Weiss, C. T. (2017). Books are forever: Early life conditions, education and lifetime earnings in Europe. The Economic Journal, 127(600), 271-296.

Carpenter, T. P. (1980). Solving verbal problems: Results and implications from national assessment. Arithmetic teacher, 28(1), 8-12.

Carraher, T. N., Carraher, D. W., \& Schliemann, A. D. (1987). Written and oral mathematics. Journal for Research in Mathematics Education, 18, 83-97.

Ceci, S. J. (1990). On intelligence ... more or less: A bio-ecological treatise on intellectual development. Englewood Cliffs, NJ: Prentice Hall.

Ceci, S. J. (1993). Some contextual trends in cognitive development. Developmental Review, 13, 403-435.

Chaby, L. E., Sheriff, M. J., Cavigelli, S. A., Hirrlinger, A. M., Lim, J., \& Braithwaite, V. A. (2016). Stress during adolescence shapes performance in adulthood: Context-dependent effects on foraging and vigilance. Ethology, 122, 284-297.

Chipman, S. F., Marshall, S. P., \& Scott, P. A. (1991). Content effects on word problem performance: A possible source of test bias? American Educational Research Journal, 28(4), 897-915.

Coxhead, A. (2000). A new academic word list. TESOL quarterly, 34(2), 213-238.

Croizet, J. C., \& Claire, T. (1998). Extending the concept of stereotype threat to social class: The intellectual underperformance of students from low socioeconomic backgrounds. Personality and Social Psychology Bulletin, 24(6), 588-594. 
Ellis, B. J., Bianchi, J., Griskevicius, V., \& Frankenhuis, W. E. (2017). Beyond risk and protective factors: An adaptation-based approach to resilience. Perspectives on Psychological Science, 12, 561-587.

Ellis, B. J., \& Del Giudice, M. (2014). Beyond allostatic load: Rethinking the role of stress in regulating human development. Development and Psychopathology, 26, 1-20.

Frankenhuis, W. E., Panchanathan, K., \& Nettle, D. (2016). Cognition in harsh and unpredictable environments. Current Opinion in Psychology, 7, 76-80.

Frankenhuis, W. E., \& de Weerth, C. (2013). Does early-life exposure to stress shape or impair cognition? Current Directions in Psychological Science, 22, 407-412.

Gable, P. A., \& Harmon-Jones, E. (2008). Approach-motivated positive affect reduces breadth of attention. Psychological Science, 19(5), 476-482.

Haag, N., Heppt, B., Stanat, P., Kuhl, P., \& Pant, H. A. (2013). Second language learners' performance in mathematics: Disentangling the effects of academic language features. Learning and Instruction, 28, 24-34.

Hart, B., \& Risley, T. R. (1995). Meaningful differences in the everyday experience of young American children. Paul H Brookes Publishing, Baltimore.

Heberle, A., \& Carter, A. (2015). Cognitive aspects of young students' experiences of economic disadvantage. Psychological Bulletin, 114, 723-746.

Kraus, M. W., Horberg, E. J., Goetz, J. L., \& Keltner, D. (2011). Social class rank, threat vigilance, and hostile reactivity. Personality and Social Psychology Bulletin, 37, 1376-1388.

Kraus, M. W., Piff, P. K., Mendoza-Denton, R., Rheinschmidt, M. L., \& Keltner, D. (2012). Social class, solipsism, and contextualism: How the rich are different from the poor. Psychological Review, 119(3), 546.

Leder, G. C., \& Forgasz, H. J. (2018). Measuring who counts: gender and mathematics assessment. ZDM Mathematics Education, 40 (4), 687-697.

McHugh, M. L. (2012). Interrater reliability: the kappa statistic. Biochemia medica, 22(3), 276282.

Magnuson, K. A., Sexton, H. R., Davis-Kean, P. E., \& Huston, A. C. (2009). Increases in maternal education and young children's language skills. Merrill-Palmer Quarterly, 55, 319-350.

Mani, A., Mullainathan, S., Shafir, E., \& Zhao, J. (2013). Poverty impedes cognitive function. Science, 341, 976-980.

Mullainathan, S., \& Sharif, E. (2013). Scarcity: Why having too little means so much. London: Allen Lane.

Murphy, M. C., Steele, C. M., \& Gross, J. J. (2007). Signaling threat: How situational cues affect women in math, science, and engineering settings. Psychological Science, 18(10), 879-885.

Murphy, M. C., \& Taylor, V. J. (2012). The role of situational cues in signaling and maintaining stereotype threat. In M. Inzlicht \& T. Schmader (Eds.), Stereotype threat: Theory, process, and application (pp. 17-33). New York, NY, US: Oxford University Press.

Nunes, T., Schliemann, A. D., \& Carraher, D. W. (1993). Mathematics in the streets and in schools. Cambridge, UK: Cambridge Univ. Press.

Nguyen, H. H. D., \& Ryan, A. M. (2008). Does stereotype threat affect test performance of minorities and women? A meta-analysis of experimental evidence. Journal of applied psychology, 93(6), 1314 . 
Olson, J. F., Martin, M. O., \& Mullis, I. V. S. (Eds.). (2008). TIMSS technical report 2007. Chestnut Hill, MA: TIMSS \& PIRLS International Study Center, Boston College.

Purpura, D. J., \& Reid, E. E. (2016). Mathematics and language: Individual and group differences in mathematical language skills in young children. Early Childhood Research Quarterly, 36, 259-268.

Ramani, G. B., Zippert, E., Schweitzer, S., \& Pan, S. (2014). Preschool children's joint block building during a guided play activity. Journal of Applied Developmental Psychology, 35(4), 326-336.

Rogers, H. J., \& Swaminathan, H. (1993). A comparison of logistic regression and MantelHaenszel procedures for detecting differential item functioning. Applied Psychological Measurement, 17(2), 105-116.

Rogoff, B., Coppens, A. D., Alcalá, L., Aceves-Azuara, I., Ruvalcaba, O., López, A., \& Dayton, A. (2017). Noticing learners' strengths through cultural research. Perspectives on Psychological Science, 12, 876-888.

Sappington, J., Larsen, C., Martin, J., \& Murphy, K. (1991). Sex differences in math problem solving as a function of gender-specific item content. Educational and psychological measurement, 51(4), 1041-1048.

Schliemann, A. D., \& Carraher, D. W. (2002). The evolution of mathematical reasoning: Everyday versus idealized understandings. Developmental Review, 22(2), 242-266.

Scheuneman, J. D., \& Grima, A. (1997). Characteristics of quantitative word items associated with differential performance for female and black examinees. Applied measurement in education, 10(4), 299-319.

Sirin, S. R. (2005). Socioeconomic status and academic achievement: A meta-analytic review of research. Review of Educational Research, 75(3), 417-453.

Spencer, S. J., Logel, C., \& Davies, P. G. (2016). Stereotype threat. Annual review of psychology, $67,415-437$

Stark, S., Chernyshenko, O. S., \& Drasgow, F. (2006). Detecting differential item functioning with confirmatory factor analysis and item response theory: Toward a unified strategy. Journal of Applied Psychology, 91(6), 1292-1306.

Sternberg, R. J. (2005). The theory of successful intelligence. Interamerican Journal of Psychology, 39(2), 189-202.

Sternberg, R. J. (2014). Teaching about the nature of intelligence. Intelligence, 42, 176-179.

Swaminathan, H., \& Rogers, H. J. (1990). Detecting differential item functioning using logistic regression procedures. Journal of Educational measurement, 27(4), 361-370.

Torney-Purta, J., Lehmann, R., Oswald, H., \& Schulz, W. (2001). Citizenship and education in twenty-eight countries: Civic knowledge and engagement at age fourteen. Amsterdam: International Association for the Evaluation of Educational Achievement.

Vandermaas-Peeler, M., Nelson, J., Bumpass, C., \& Sassine, B. (2009). Numeracy-related exchanges in joint storybook reading and play. International Journal of Early Years Education, 17(1), 67-84.

Valencia, R. R. (2010). Dismantling contemporary deficit thinking: Educational thought and practice. New York, NY: Routledge.

Varnum, M. E., Grossmann, I., Kitayama, S., \& Nisbett, R. E. (2010). The origin of cultural differences in cognition: The social orientation hypothesis. Current Directions in Psychological Science, 19(1), 9-13. 
Wagenmakers, E. J., Wetzels, R., Borsboom, D., van der Maas, H. J. L., \& Kievit, R. A. (2012). An agenda for purely confirmatory research. Perspectives on Psychological Science, 7 , 632-638.

Walker, C. M. (2011). What's the DIF? Why differential item functioning analyses are an important part of instrument development and validation. Journal of Psychoeducational Assessment, 29(4), 364-376.

Walton, G. M., \& Spencer, S. J. (2009). Latent ability: Grades and test scores systematically underestimate the intellectual ability of negatively stereotyped students. Psychological Science, 20(9), 1132-1139.

Warne, R. T., Yoon, M., \& Price, C. J. (2014). Exploring the various interpretations of "test bias". Cultural Diversity and Ethnic Minority Psychology, 20(4), 570-582.

Yang, Y., \& Gustafsson, J. E. (2004). Measuring socioeconomic status at individual and collective levels. Educational Research and Evaluation, 10(3), 259-288.

Zohar, A., \& Gershikov, A. (2008). Gender and performance in mathematical tasks: Does the context make a difference? International Journal of Science and Mathematics Education, 6(4), 677-693. 


\subsection{Appendix}

TABLE A1 Selected Math Items

\begin{tabular}{|c|c|c|c|c|c|c|}
\hline \multicolumn{4}{|c|}{ TIMSS item information } & \multicolumn{3}{|c|}{ Low-SES familiar categories } \\
\hline Number & Grade & $\begin{array}{l}\text { Context } \\
\text { domain }\end{array}$ & $\begin{array}{l}\text { Cognitive } \\
\text { domain }\end{array}$ & $\begin{array}{l}\text { Low-SES } \\
\text { Category }\end{array}$ & $\begin{array}{l}\text { Low-SES } \\
\text { familiar } \\
\text { context }\end{array}$ & Low-SES familiar Problem \\
\hline 1 & 4 & Number & Applying & Resources & Money, lunch & Money left after paying for lunch \\
\hline 2 & 4 & $\begin{array}{l}\text { Data } \\
\text { display }\end{array}$ & Knowing & Resources & Money, rent & Cost of renting a bike \\
\hline 3 & 4 & Number & Reasoning & Resources & $\begin{array}{l}\text { Money, } \\
\text { fair }\end{array}$ & Ticket price for a fair \\
\hline 4 & 4 & Geometry & Knowing & Resources & Market, shop & Finding the location of a shop \\
\hline 5 & 4 & Number & Knowing & Resources & Cake & $\begin{array}{l}\text { Computing how much of a cake } \\
\text { was eaten }\end{array}$ \\
\hline 6 & 8 & Number & Applying & Resources & $\begin{array}{l}\text { Prize for a } \\
\text { coat }\end{array}$ & $\begin{array}{l}\text { Amount of money saved after } \\
\text { reduction of } 30 \%\end{array}$ \\
\hline 7 & 8 & Algebra & Reasoning & Resources & $\begin{array}{l}\text { Prize for } \\
\text { pencils }\end{array}$ & Money needed to buy pencils \\
\hline 8 & 8 & $\begin{array}{l}\text { Data and } \\
\text { Chance }\end{array}$ & Reasoning & Resources & Costs of a trip & $\begin{array}{l}\text { Travel possibilities given certain } \\
\text { (cost) restrictions }\end{array}$ \\
\hline 9 & 8 & Number & Applying & Resources & Money & $\begin{array}{l}\text { Results of unequal distribution of } \\
\text { money between two persons }\end{array}$ \\
\hline 10 & 8 & $\begin{array}{l}\text { Data and } \\
\text { Chance }\end{array}$ & Knowing & Resources & Candies & Likelihood of getting a candy \\
\hline 11 & 4 & $\begin{array}{l}\text { Data } \\
\text { display }\end{array}$ & Applying & $\begin{array}{l}\text { Social } \\
\text { interaction }\end{array}$ & Family & $\begin{array}{l}\text { Counting classmates' brothers and } \\
\text { sisters }\end{array}$ \\
\hline 12 & 4 & Number & Reasoning & $\begin{array}{l}\text { Social } \\
\text { interaction }\end{array}$ & $\begin{array}{l}\text { Running } \\
\text { competition }\end{array}$ & $\begin{array}{l}\text { Computing how far the winner } \\
\text { had run }\end{array}$ \\
\hline 13 & 4 & Number & Reasoning & $\begin{array}{l}\text { Social } \\
\text { interaction }\end{array}$ & Game & $\begin{array}{l}\text { Discovering a rule that participants } \\
\text { use }\end{array}$ \\
\hline 14 & 4 & Number & Applying & $\begin{array}{l}\text { Social } \\
\text { interaction }\end{array}$ & $\begin{array}{l}\text { Trading with } \\
\text { cards }\end{array}$ & $\begin{array}{l}\text { Computing how many cards stu- } \\
\text { dents can get in a trading game }\end{array}$ \\
\hline 15 & 4 & Number & Reasoning & $\begin{array}{l}\text { Social } \\
\text { interaction }\end{array}$ & Lottery & $\begin{array}{l}\text { Reasoning about the prize-winning } \\
\text { numbers }\end{array}$ \\
\hline 16 & 4 & Number & Reasoning & $\begin{array}{l}\text { Social } \\
\text { interaction }\end{array}$ & Sharing & $\begin{array}{l}\text { Amount of sweets needed to share } \\
\text { them equally between students }\end{array}$ \\
\hline 17 & 8 & Number & Knowing & $\begin{array}{l}\text { Social } \\
\text { interaction }\end{array}$ & Basketball & $\begin{array}{l}\text { Number and percentage of success- } \\
\text { ful shots }\end{array}$ \\
\hline 18 & 8 & $\begin{array}{l}\text { Data and } \\
\text { Chance }\end{array}$ & Reasoning & $\begin{array}{l}\text { Social } \\
\text { interaction }\end{array}$ & $\begin{array}{l}\text { Long jump } \\
\text { competition }\end{array}$ & $\begin{array}{l}\text { Interpretation of the results: who } \\
\text { won the competition }\end{array}$ \\
\hline 19 & 8 & $\begin{array}{l}\text { Data and } \\
\text { Chance }\end{array}$ & Knowing & $\begin{array}{l}\text { Social } \\
\text { interaction }\end{array}$ & $\begin{array}{l}\text { Elections } \\
\text { for school } \\
\text { president }\end{array}$ & $\begin{array}{l}\text { Likelihood that a single student } \\
\text { voted on a certain candidate }\end{array}$ \\
\hline 20 & 8 & $\begin{array}{l}\text { Data and } \\
\text { Chance }\end{array}$ & Reasoning & $\begin{array}{l}\text { Social } \\
\text { interaction }\end{array}$ & $\begin{array}{l}\text { Taking care of } \\
\text { elderly }\end{array}$ & $\begin{array}{l}\text { Age distribution of a population } \\
\text { and problems with taking care of } \\
\text { elderly }\end{array}$ \\
\hline
\end{tabular}


TABLE A2 Results from DIF-Analyses on Items with Low-SES familiar context

\begin{tabular}{|c|c|c|c|c|c|c|}
\hline \multicolumn{3}{|l|}{ Items } & \multicolumn{4}{|l|}{ Results } \\
\hline Number & $\begin{array}{l}\text { Low-SES } \\
\text { Category }\end{array}$ & $\begin{array}{l}\text { Low-SES familiar } \\
\text { context }\end{array}$ & $\begin{array}{l}\text { LR } \\
\operatorname{Exp}(B)\end{array}$ & $\begin{array}{l}\text { LR } \\
95 \% \mathrm{Cl}\end{array}$ & MH & DIF \\
\hline 1 & Resources & Money, fair & $0.670^{\star *}$ & {$[0.543,0.803]$} & Flag A & $\begin{array}{l}\text { DIF, disadvantage for } \\
\text { low-SES }\end{array}$ \\
\hline 2 & Resources & Market, shop & $0.885^{\star *}$ & {$[0.778,0.991]$} & Flag A & $\begin{array}{l}\text { DIF, disadvantage for } \\
\text { low-SES }\end{array}$ \\
\hline 3 & Resources & Prize for a coat & $0.836^{\star \star}$ & {$[0.757,0.915]$} & Flag A & $\begin{array}{l}\text { DIF, disadvantage for } \\
\text { low-SES }\end{array}$ \\
\hline 4 & Resources & Prize for pencils & $0.710^{\star \star}$ & {$[0.607,0.812]$} & Flag A & $\begin{array}{l}\text { DIF, disadvantage for } \\
\text { low-SES }\end{array}$ \\
\hline 5 & Resources & Candies & $0.775^{\star \star}$ & {$[0.704,0.846]$} & Flag A & $\begin{array}{l}\text { DIF, disadvantage for } \\
\text { low-SES }\end{array}$ \\
\hline 6 & Resources & Money, lunch & 1.022 & {$[0.907,1.137]$} & . & \\
\hline 7 & Resources & Money, rent & 0.956 & {$[0.818,1.094]$} & . & \\
\hline 8 & Resources & Cake & 1.076 & {$[0.942,1.21]$} & . & \\
\hline 9 & Resources & Costs of a trip & 0.963 & {$[0.848,1.077]$} & . & \\
\hline 10 & Resources & Money & 1.015 & {$[0.888,1.141]$} & . & \\
\hline 11 & Social interaction & Lottery & $0.659^{\star \star}$ & {$[0.574,0.743]$} & Flag A & $\begin{array}{l}\text { DIF, disadvantage for } \\
\text { low-SES }\end{array}$ \\
\hline 12 & Social interaction & $\begin{array}{l}\text { Long jump compe- } \\
\text { tition }\end{array}$ & $0.817^{\star \star}$ & {$[0.743,0.89]$} & Flag A & $\begin{array}{l}\text { DIF, disadvantage for } \\
\text { low-SES }\end{array}$ \\
\hline 13 & Social interaction & $\begin{array}{l}\text { Elections for school } \\
\text { president }\end{array}$ & $0.581^{* \star}$ & {$[0.525,0.637]$} & Flag A & $\begin{array}{l}\text { DIF, disadvantage for } \\
\text { low-SES }\end{array}$ \\
\hline 14 & Social interaction & Taking care of elderly & $0.710^{\star \star}$ & {$[0.607,0.812]$} & Flag A & $\begin{array}{l}\text { DIF, disadvantage for } \\
\text { low-SES }\end{array}$ \\
\hline 15 & Social interaction & Family & $0.882^{*}$ & {$[0.768,0.996]$} & . & \\
\hline 16 & Social interaction & Running competition & 0.968 & {$[0.834,1.102]$} & . & \\
\hline 17 & Social interaction & Game & 0.995 & {$[0.839,1.15]$} & . & \\
\hline 18 & Social interaction & Trading with cards & 1.062 & {$[0.943,1.181]$} & . & \\
\hline 19 & Social interaction & Sharing & 0.935 & {$[0.811,1.058]$} & . & \\
\hline 20 & Social interaction & Basketball & 1.039 & {$[0.926,1.151]$} & . & \\
\hline
\end{tabular}

Note: Flag A = small levels of DIF. Items sorted by low-SES category and DIF. 
TABLE A3 Selected Math Items with Low-SES Neutral Context and Problems

\begin{tabular}{|c|c|c|c|c|c|c|}
\hline \multicolumn{4}{|c|}{ TIMSS item information } & \multicolumn{3}{|c|}{ Low-SES familiar categories } \\
\hline Number & Grade & $\begin{array}{l}\text { Context } \\
\text { domain }\end{array}$ & $\begin{array}{l}\text { Cognitive } \\
\text { domain }\end{array}$ & Problem & $\begin{array}{l}\text { Low-SES } \\
\text { neutral Context }\end{array}$ & $\begin{array}{l}\text { Low-SES neutral } \\
\text { Problem }\end{array}$ \\
\hline 21 & 4 & Geometry & Knowing & $\begin{array}{l}\text { Word } \\
\text { problem }\end{array}$ & $\begin{array}{l}\text { Pieces of card- } \\
\text { board }\end{array}$ & $\begin{array}{l}\text { Making shapes without } \\
\text { cutting the cardboard }\end{array}$ \\
\hline 22 & 4 & Number & Applying & $\begin{array}{l}\text { Word } \\
\text { problem }\end{array}$ & Matches & $\begin{array}{l}\text { Amount of matches } \\
\text { needed to form a figure }\end{array}$ \\
\hline 23 & 4 & Geometry & Applying & $\begin{array}{l}\text { Word } \\
\text { problem }\end{array}$ & Boxes in a corner & $\begin{array}{l}\text { Counting number of } \\
\text { boxes }\end{array}$ \\
\hline 24 & 4 & Number & Reasoning & $\begin{array}{l}\text { Word } \\
\text { problem }\end{array}$ & Temperature & $\begin{array}{l}\text { Computing increase in } \\
\text { temperature }\end{array}$ \\
\hline 25 & 4 & Number & Applying & $\begin{array}{l}\text { Word } \\
\text { problem }\end{array}$ & Cat & Weight of a cat \\
\hline 26 & 8 & Geometry & Applying & $\begin{array}{l}\text { Word } \\
\text { problem }\end{array}$ & Ponds and frogs & $\begin{array}{l}\text { Amount of frogs in a } \\
\text { pond }\end{array}$ \\
\hline 27 & 8 & Algebra & Reasoning & $\begin{array}{l}\text { Word } \\
\text { problem }\end{array}$ & Tiles & $\begin{array}{l}\text { Making a shape with } \\
\text { black and red tiles }\end{array}$ \\
\hline 28 & 8 & Geometry & Applying & $\begin{array}{l}\text { Word } \\
\text { problem }\end{array}$ & Cubes & $\begin{array}{l}\text { Cubes needed to fill } \\
\text { a hole }\end{array}$ \\
\hline 29 & 8 & Number & Applying & $\begin{array}{l}\text { Word } \\
\text { problem }\end{array}$ & Length of a pipe & $\begin{array}{l}\text { Computing the length of } \\
\text { a pipe after cutting } 1 / 5\end{array}$ \\
\hline 30 & 8 & $\begin{array}{l}\text { Data and } \\
\text { Chance }\end{array}$ & Knowing & $\begin{array}{l}\text { Word } \\
\text { problem }\end{array}$ & Staff members & $\begin{array}{l}\text { Mean number of staff } \\
\text { members }\end{array}$ \\
\hline 31 & 4 & Geometry & Knowing & $\begin{array}{l}\text { Math } \\
\text { notation }\end{array}$ & Rotation & $\begin{array}{l}\text { Position of a shape after } \\
\text { rotation }\end{array}$ \\
\hline 32 & 4 & Number & Knowing & $\begin{array}{l}\text { Math } \\
\text { notation }\end{array}$ & Fraction & Comparing fractions \\
\hline 33 & 4 & Geometry & Applying & $\begin{array}{l}\text { Math } \\
\text { notation }\end{array}$ & Shape & Drawing a shape \\
\hline 34 & 4 & Number & Knowing & $\begin{array}{l}\text { Math } \\
\text { notation }\end{array}$ & Number & $\begin{array}{l}\text { The value of } 8 \text { in a } \\
\text { number }\end{array}$ \\
\hline 35 & 4 & Number & Applying & $\begin{array}{l}\text { Math } \\
\text { notation }\end{array}$ & Fractions & Shade $1 / 2$ of a triangle \\
\hline 36 & 8 & Number & Applying & $\begin{array}{l}\text { Math } \\
\text { notation }\end{array}$ & Fractions & Comparing fractions \\
\hline 37 & 8 & Geometry & Applying & $\begin{array}{l}\text { Math } \\
\text { notation }\end{array}$ & Figure & $\begin{array}{l}\text { Computing the area of } \\
\text { a figure }\end{array}$ \\
\hline 38 & 8 & Number & Knowing & $\begin{array}{l}\text { Math } \\
\text { notation }\end{array}$ & Fractions & Estimates of fractions \\
\hline 39 & 8 & Number & Knowing & $\begin{array}{l}\text { Math } \\
\text { notation }\end{array}$ & Prime factors & $\begin{array}{l}\text { Recognizing prime } \\
\text { factors }\end{array}$ \\
\hline 40 & 8 & Number & Knowing & $\begin{array}{l}\text { Math } \\
\text { notation }\end{array}$ & Fractions & $\begin{array}{l}\text { Writing fractions in } \\
\text { decimals }\end{array}$ \\
\hline
\end{tabular}


TABLE A4 Results from DIF-analyses on Items with low-SES Neutral Context

\begin{tabular}{|c|c|c|c|c|c|c|}
\hline \multicolumn{3}{|l|}{ Items } & \multicolumn{4}{|l|}{ Results } \\
\hline Number & Context & Problem & $\begin{array}{l}\text { LR } \\
\operatorname{Exp}(B)\end{array}$ & $\begin{array}{l}\text { LR } \\
95 \% \mathrm{Cl}\end{array}$ & MH & Decision \\
\hline 21 & $\begin{array}{l}\text { Pieces of card- } \\
\text { board }\end{array}$ & Word problem & $0.620^{\star \star}$ & {$[0.546,0.704]$} & Flag A & $\begin{array}{l}\text { DIF, disadvantage for } \\
\text { low-SES }\end{array}$ \\
\hline 22 & Matches & Word problem & 0.940 & {$[0.849,1.040]$} & . & \\
\hline 23 & Boxes in a corner & Word problem & 1.029 & {$[0.913,1.161]$} & . & \\
\hline 24 & Temperature & Word problem & $0.820^{*}$ & {$[0.720,0.933]$} & . & \\
\hline 25 & Cat & Word problem & 1.060 & {$[0.917,1.225]$} & . & \\
\hline 26 & Ponds and frogs & Word problem & 0.938 & {$[0.849,1.035]$} & . & \\
\hline 27 & Tiles & Word problem & 1.113 & {$[0.994,1.246]$} & . & \\
\hline 28 & Cubes & Word problem & $0.743^{\star \star}$ & {$[0.676,0.817]$} & Flag A & $\begin{array}{l}\text { DIF, disadvantage for } \\
\text { low-SES }\end{array}$ \\
\hline 29 & Length of a pipe & Word problem & $0.781^{\star \star}$ & {$[0.703,0.868]$} & . & \\
\hline 30 & Staff members & Word problem & $0.783^{\star \star}$ & {$[0.701,0.874]$} & . & \\
\hline 31 & Rotation & Math notation & $0.846^{*}$ & {$[0.754,0.949]$} & . & \\
\hline 32 & Fraction & Math notation & $0.831^{\star \star}$ & {$[0.760,0.909]$} & . & \\
\hline 33 & Shape & Math notation & 0.981 & {$[0.891,1.080]$} & . & \\
\hline 34 & Number & Math notation & 0.907 & {$[0.804,1.002]$} & . & \\
\hline 35 & Fractions & Math notation & 0.781 & {$[0.686,0.888]$} & . & \\
\hline 36 & Fractions & Math notation & $1.175^{*}$ & {$[1.064,1.296$} & Flag A & DIF in favor of low-SES \\
\hline 37 & Figure & Math notation & $0.865^{\star \star}$ & {$[0.785,0.954]$} & Flag A & $\begin{array}{l}\text { DIF, disadvantage for } \\
\text { low-SES }\end{array}$ \\
\hline 38 & Fractions & Math notation & $0.725^{\star \star}$ & {$[0.658,0.800]$} & Flag A & $\begin{array}{l}\text { DIF, disadvantage for } \\
\text { low-SES }\end{array}$ \\
\hline 39 & Prime factors & Math notation & 0.912 & {$[0.830,1.002]$} & . & \\
\hline 40 & Fractions & Math notation & 1.001 & {$[0.882,1.137]$} & . & \\
\hline
\end{tabular}

Note: Flag $A=$ small levels of DIF 
TABLE A5 Selected Math Items with low-SES Familiar Context, Replication with Years 1999 and 2003

\begin{tabular}{|c|c|c|c|c|c|c|}
\hline \multicolumn{4}{|c|}{ TIMSS item information } & \multicolumn{3}{|c|}{ Low-SES familiar categories } \\
\hline Number & Grade & Context domain & Cognitive domain & $\begin{array}{l}\text { Low-SES } \\
\text { Category }\end{array}$ & $\begin{array}{l}\text { Low-SES } \\
\text { familiar } \\
\text { context }\end{array}$ & $\begin{array}{l}\text { Low-SES familiar } \\
\text { Problem }\end{array}$ \\
\hline 1 & 4 & Number & $\begin{array}{l}\text { Solving routine } \\
\text { problems }\end{array}$ & Resources & Cake & $\begin{array}{l}\text { Computing how much } \\
\text { cake is left }\end{array}$ \\
\hline 2 & 8 & $\begin{array}{l}\text { Data representa- } \\
\text { tion, analysis and } \\
\text { probability }\end{array}$ & $\begin{array}{l}\text { Communicating and } \\
\text { reasoning }\end{array}$ & Resources & $\begin{array}{l}\text { Costs of } \\
\text { magazines }\end{array}$ & $\begin{array}{l}\text { Decide which } \\
\text { magazine is the least } \\
\text { expensive }\end{array}$ \\
\hline 3 & 8 & $\begin{array}{l}\text { Fraction and } \\
\text { number sense }\end{array}$ & $\begin{array}{l}\text { Investigating and } \\
\text { solving problems }\end{array}$ & Resources & Food & $\begin{array}{l}\text { Calories in portion } \\
\text { of food }\end{array}$ \\
\hline 4 & 4 & Number & $\begin{array}{l}\text { Solving routine } \\
\text { problems }\end{array}$ & Resources & Food & $\begin{array}{l}\text { Estimating how many } \\
\text { cabbages there are }\end{array}$ \\
\hline 5 & 4 & Algebra & Using concepts & Resources & $\begin{array}{l}\text { Selling } \\
\text { apples }\end{array}$ & $\begin{array}{l}\text { How many apples } \\
\text { are left }\end{array}$ \\
\hline 6 & 4 & Number & $\begin{array}{l}\text { Solving routine } \\
\text { problems }\end{array}$ & Resources & Cake & Fraction of cake eaten \\
\hline 7 & 4 & Data & Using concepts & Resources & Ice-cream & $\begin{array}{l}\text { Flavor of favorite } \\
\text { ice-cream }\end{array}$ \\
\hline 8 & 8 & $\begin{array}{l}\text { Fractions and } \\
\text { number sense }\end{array}$ & $\begin{array}{l}\text { Investigating and } \\
\text { solving problems }\end{array}$ & Resources & Trading & $\begin{array}{l}\text { Money received } \\
\text { during trading }\end{array}$ \\
\hline 9 & 8 & Algebra & Knowing & Resources & Money & Costs of printed cards \\
\hline 10 & 8 & $\begin{array}{l}\text { Fractions and } \\
\text { number sense }\end{array}$ & $\begin{array}{l}\text { Investigating and } \\
\text { solving problems }\end{array}$ & Resources & Money & $\begin{array}{l}\text { Money left after } \\
\text { spending money }\end{array}$ \\
\hline 11 & 4 & Data & $\begin{array}{l}\text { Solving routine } \\
\text { problems }\end{array}$ & $\begin{array}{l}\text { Social inter- } \\
\text { action }\end{array}$ & Trading & $\begin{array}{l}\text { Compute how many } \\
\text { pencils were sold }\end{array}$ \\
\hline 12 & 4 & Data & Reasoning & $\begin{array}{l}\text { Social inter- } \\
\text { action }\end{array}$ & $\begin{array}{l}\text { Comparison } \\
\text { of persons }\end{array}$ & $\begin{array}{l}\text { Comparing person's } \\
\text { length }\end{array}$ \\
\hline 13 & 4 & Number & Using concepts & $\begin{array}{l}\text { Social inter- } \\
\text { action }\end{array}$ & Game & How to get 20 points \\
\hline 14 & 4 & Number & $\begin{array}{l}\text { Solving routine } \\
\text { problems }\end{array}$ & $\begin{array}{l}\text { Social inter- } \\
\text { action }\end{array}$ & $\begin{array}{l}\text { Sharing } \\
\text { sweets }\end{array}$ & $\begin{array}{l}\text { How to share sweets } \\
\text { equally }\end{array}$ \\
\hline 15 & 8 & $\begin{array}{l}\text { Data representa- } \\
\text { tion, analysis and } \\
\text { probability }\end{array}$ & $\begin{array}{l}\text { Using complex } \\
\text { procedures }\end{array}$ & $\begin{array}{l}\text { Social inter- } \\
\text { action }\end{array}$ & Trading & $\begin{array}{l}\text { Determine when the } \\
\text { greatest increase in } \\
\text { sales occur }\end{array}$ \\
\hline 16 & 8 & Measurement & $\begin{array}{l}\text { Investigating and } \\
\text { solving problems }\end{array}$ & $\begin{array}{l}\text { Social inter- } \\
\text { action }\end{array}$ & $\begin{array}{l}\text { Comparing } \\
\text { paces }\end{array}$ & $\begin{array}{l}\text { Comparing how many } \\
\text { paces persons have } \\
\text { to take }\end{array}$ \\
\hline 17 & 8 & $\begin{array}{l}\text { Fraction and } \\
\text { number sense }\end{array}$ & $\begin{array}{l}\text { Investigating and } \\
\text { solving problems }\end{array}$ & $\begin{array}{l}\text { Social inter- } \\
\text { action }\end{array}$ & $\begin{array}{l}\text { Sharing } \\
\text { marbles }\end{array}$ & $\begin{array}{l}\text { Computing how many } \\
\text { marbles are available }\end{array}$ \\
\hline 18 & 8 & $\begin{array}{l}\text { Fraction and } \\
\text { number sense }\end{array}$ & $\begin{array}{l}\text { Investigating and } \\
\text { solving problems }\end{array}$ & $\begin{array}{l}\text { Social inter- } \\
\text { action }\end{array}$ & $\begin{array}{l}\text { Sharing } \\
\text { cherries }\end{array}$ & $\begin{array}{l}\text { Number of cherries } \\
\text { left after sharing }\end{array}$ \\
\hline 19 & 8 & $\begin{array}{l}\text { Fraction and } \\
\text { number sense }\end{array}$ & $\begin{array}{l}\text { Investigating and } \\
\text { solving problems }\end{array}$ & $\begin{array}{l}\text { Social inter- } \\
\text { action }\end{array}$ & Game & $\begin{array}{l}\text { Computing the } \\
\text { average speed of a } \\
\text { runner }\end{array}$ \\
\hline 20 & 4 & Data & $\begin{array}{l}\text { Solving routine } \\
\text { problems }\end{array}$ & $\begin{array}{l}\text { Social inter- } \\
\text { action }\end{array}$ & $\begin{array}{l}\text { Comparison } \\
\text { between } \\
\text { classes }\end{array}$ & $\begin{array}{l}\text { Comparing perfor- } \\
\text { mance of classes }\end{array}$ \\
\hline
\end{tabular}


TABLE A6 Results from DIF-analyses on Items with low-SES Familiar Context, Replication with Years 1999 and 2003

\begin{tabular}{|c|c|c|c|c|c|c|}
\hline \multicolumn{3}{|l|}{ Items } & \multicolumn{4}{|l|}{ Results } \\
\hline Number & $\begin{array}{l}\text { Low-SES Low-SE } \\
\text { Category }\end{array}$ & S familiar context & $\begin{array}{l}\text { LR } \\
\operatorname{Exp}(B)\end{array}$ & $\begin{array}{l}\text { LR } \\
95 \% \mathrm{Cl}\end{array}$ & $\mathrm{MH}$ & Decision \\
\hline 1 & Resources & Cake & $0.801^{\star \star}$ & $\begin{array}{l}{[0.708,} \\
0.908]\end{array}$ & Flag A & $\begin{array}{l}\text { DIF, disadvantage for } \\
\text { low-SES }\end{array}$ \\
\hline 2 & Resources & $\begin{array}{l}\text { Costs of } \\
\text { magazines }\end{array}$ & $0.494^{\star *}$ & $\begin{array}{l}{[0.447,} \\
0.547]\end{array}$ & Flag A & $\begin{array}{l}\text { DIF, disadvantage for } \\
\text { low-SES }\end{array}$ \\
\hline 3 & Resources & Food & 0.945 & $\begin{array}{l}{[0.891,} \\
1.001]\end{array}$ & Flag A & \\
\hline 4 & Resources & Food & 1.053 & $\begin{array}{l}\text { [0.931, } \\
1.191]\end{array}$ & . & \\
\hline 5 & Resources & Selling apples & 0.863 & $\begin{array}{l}{[0.868,} \\
1.184]\end{array}$ & . & \\
\hline 6 & Resources & Cake & 0.875 & $\begin{array}{l}{[0.761,} \\
1.006]\end{array}$ & . & \\
\hline 7 & Resources & Ice-cream & $0.830^{\star \star}$ & $\begin{array}{l}{[0.730,} \\
0.934]\end{array}$ & . & \\
\hline 8 & Resources & Trading & 0.897 & $\begin{array}{l}{[0.787,} \\
1.002]\end{array}$ & . & \\
\hline 9 & Resources & Money & 0.962 & $\begin{array}{l}{[0.904,} \\
1.023]\end{array}$ & . & \\
\hline 10 & Resources & Money & 1.117 & $\begin{array}{l}{[0.972,} \\
1.283]\end{array}$ & . & \\
\hline 11 & Social interaction & Trading & $0.631^{\star \star}$ & $\begin{array}{l}{[0.549,} \\
0.725]\end{array}$ & Flag A & $\begin{array}{l}\text { DIF, disadvantage for } \\
\text { low-SES }\end{array}$ \\
\hline 12 & Social interaction & $\begin{array}{l}\text { Comparison of } \\
\text { persons' length }\end{array}$ & $0.697^{\star \star}$ & $\begin{array}{l}{[0.609,} \\
0.797]\end{array}$ & Flag A & $\begin{array}{l}\text { DIF, disadvantage for } \\
\text { low-SES }\end{array}$ \\
\hline 13 & Social interaction & Game & $0.691^{\star \star}$ & $\begin{array}{l}{[0.551} \\
0.866]\end{array}$ & Flag A & $\begin{array}{l}\text { DIF, disadvantage for } \\
\text { low-SES }\end{array}$ \\
\hline 14 & Social interaction & Sharing sweets & $0.698^{\star \star}$ & $\begin{array}{l}{[0.579} \\
0.842]\end{array}$ & Flag A & $\begin{array}{l}\text { DIF, disadvantage for } \\
\text { low-SES }\end{array}$ \\
\hline 15 & Social interaction & Trading & $0.556^{\star \star}$ & $\begin{array}{l}\text { [0.525, } \\
0.588]\end{array}$ & Flag A & $\begin{array}{l}\text { DIF, disadvantage for } \\
\text { low-SES }\end{array}$ \\
\hline 16 & Social interaction & Comparing paces & $0.459^{\star \star}$ & $\begin{array}{l}{[0.408,} \\
0.517]\end{array}$ & Flag A & $\begin{array}{l}\text { DIF, disadvantage for } \\
\text { low-SES }\end{array}$ \\
\hline 17 & Social interaction & Sharing marbles & 1.026 & $\begin{array}{l}{[0.921,} \\
1.143]\end{array}$ & . & \\
\hline 18 & Social interaction & Sharing cherries & 0.912 & $\begin{array}{l}{[0.821} \\
1.024]\end{array}$ & . & \\
\hline 19 & Social interaction & Game & 1.006 & $\begin{array}{l}{[0.939,} \\
1.079]\end{array}$ & . & \\
\hline 20 & Social interaction & $\begin{array}{l}\text { Comparison } \\
\text { between classes }\end{array}$ & 0.870 & $\begin{array}{l}{[0.707,} \\
1.070]\end{array}$ & . & \\
\hline
\end{tabular}

Note. Flag $A=$ small levels of DIF. Items sorted by low-SES category and DIF. 
TABLE A7 Results from Logistic Repeated Measures Analyses Predicting Change of Giving the Correct Answer among Students in Grade 4 from Different SES-Backgrounds by Relevance of Context of Items

\begin{tabular}{|c|c|c|}
\hline & $\operatorname{Exp}(B)$ & $95 \% \mathrm{Cl}$ \\
\hline \multicolumn{3}{|l|}{ SES (scale 1-5, SES $5=$ ref) } \\
\hline SES 1 & $1.03^{\star \star}$ & {$[1.02,1.04]$} \\
\hline SES 2 & $1.01^{\star \star}$ & {$[1.00,1.02]$} \\
\hline $\mathrm{SES}_{3}$ & 1.00 & {$[0.99,1.01]$} \\
\hline $\mathrm{SES}_{4}$ & 1.00 & {$[0.99,1.01]$} \\
\hline \multicolumn{3}{|l|}{ Context category (Neutral = ref) } \\
\hline Low-SES familiar & $1.48^{\star \star}$ & {$[1.45,1.51]$} \\
\hline \multicolumn{3}{|c|}{ Interaction SES x Context category (SES 5 = ref) } \\
\hline SES $1 \times$ Low-SES familiar & $0.82^{* \star}$ & {$[0.80,0.85]$} \\
\hline SES $2 \times$ Low-SES familiar & $0.89^{\star \star}$ & {$[0.86,0.91]$} \\
\hline SES $3 \times$ Low-SES familiar & $0.95^{* *}$ & {$[0.93,0.98]$} \\
\hline SES $4 \times$ Low-SES familiar & 0.98 & {$[0.95,1.01]$} \\
\hline \multicolumn{3}{|l|}{ Math ability } \\
\hline Average performance & $141.54^{\star \star}$ & {$[140.39,142.71]$} \\
\hline \multicolumn{3}{|l|}{ Word problem $($ no $=$ ref) } \\
\hline Word problem & $0.67^{\star \star}$ & {$[0.67,0.68]$} \\
\hline \multicolumn{3}{|l|}{ Item-type $(C R=$ ref $)$} \\
\hline Multiple Choice & $1.25^{\star *}$ & {$[1.24,1.26]$} \\
\hline \multicolumn{3}{|l|}{ Context domain item (number $=$ ref) } \\
\hline Data Display & $2.57^{\star \star}$ & {$[2.54,2.60]$} \\
\hline Geometric shapes and measures & $1.42^{\star \star}$ & {$[1.41,1.43]$} \\
\hline \multicolumn{3}{|l|}{ Cognitive domain item (reasoning = ref) } \\
\hline Applying & $2.24^{\star *}$ & {$[2.22,2.26]$} \\
\hline Knowing & $1.75^{\star \star}$ & {$[1.73,1.77]$} \\
\hline \multicolumn{3}{|l|}{ General language features } \\
\hline Total word count & $1.03^{\star *}$ & {$[1.03,1.03]$} \\
\hline Number of different words & $0.99^{\star \star}$ & {$[0.99,0.99]$} \\
\hline Total number of characters & $1.01^{\star \star}$ & {$[1.01,1.01]$} \\
\hline Number of characters without spaces & $0.99^{\star \star}$ & {$[0.99,0.99]$} \\
\hline Average syllables per Word & $0.78^{\star \star}$ & {$[0.77,0.79]$} \\
\hline Sentence count & $0.95^{\star \star}$ & {$[0.95,0.95]$} \\
\hline Averages sentence length in words & $0.95^{\star *}$ & {$[0.95,0.95]$} \\
\hline Academic word use (AWL) & $0.95^{\star \star}$ & {$[0.74,0.75]$} \\
\hline \multicolumn{3}{|l|}{ Math language features } \\
\hline Quantitative language & $1.42^{\star \star}$ & {$[1.41,1.43]$} \\
\hline Spatial language & $0.77^{\star \star}$ & {$[0.77,0.78]$} \\
\hline Intercept & $0.05^{\star \star}$ & {$[0.05,0.06]$} \\
\hline $\mathrm{N}$ & 2779383 & \\
\hline
\end{tabular}

Note. $\mathrm{Cl}=$ confidence interval, ${ }^{*} p<.01,{ }^{* *} p<.001$. Country was coded as 57 dummy-variables, and included as covariate in our model (results not shown). 
TABLE A8 Results from Logistic Repeated Measures Analyses Predicting Change of Giving the Correct Answer among Students in Grade 8 from Different SES-Backgrounds by Relevance of Context of Items

\begin{tabular}{|c|c|c|}
\hline & $\operatorname{Exp}(B)$ & $95 \% \mathrm{Cl}$ \\
\hline \multicolumn{3}{|l|}{ SES (scale 1-5, SES $5=$ ref) } \\
\hline SES 1 & $1.02^{\star \star}$ & {$[1.02,1.03]$} \\
\hline SES 2 & $1.03^{\star \star}$ & {$[1.02,1.03]$} \\
\hline $\mathrm{SES}_{3}$ & $1.01^{*}$ & {$[1.01,1.02]$} \\
\hline $\mathrm{SES}_{4}$ & 0.99 & {$[0.99,1.01]$} \\
\hline \multicolumn{3}{|l|}{ Context category (Neutral = ref) } \\
\hline Low-SES familiar & $1.28^{\star \star}$ & {$[1.25,1.30]$} \\
\hline \multicolumn{3}{|c|}{ Interaction SES $x$ Context category $\left(S_{E S} 5=\right.$ ref) } \\
\hline SES $1 \times$ Low-SES familiar & $0.84^{\star \star}$ & {$[0.84,0.88]$} \\
\hline SES $2 \times$ Low-SES familiar & $0.85^{\star *}$ & {$[0.85,0.89]$} \\
\hline SES $_{3} \times$ Low-SES familiar & $0.89^{\star *}$ & {$[0.89,0.93]$} \\
\hline SES $4 \times$ Low-SES familiar & $0.94^{*}$ & {$[0.94,0.99]$} \\
\hline \multicolumn{3}{|l|}{ Math ability } \\
\hline Average performance & $154.06^{\star \star}$ & {$[152.90,155.22]$} \\
\hline \multicolumn{3}{|l|}{ Word problem $($ no $=$ ref) } \\
\hline Word problem & $1.39^{\star \star}$ & {$[1.38,1.41]$} \\
\hline \multicolumn{3}{|l|}{ Item type (open question = ref) } \\
\hline Multiple choice & $1.66^{\star \star}$ & {$[1.64,1.67]$} \\
\hline \multicolumn{3}{|l|}{ Context domain item (number = ref) } \\
\hline Algebra & $0.76^{\star \star}$ & {$[0.75,0.77]$} \\
\hline Data and chance & $2.00^{\star \star}$ & {$[1.98,2.02]$} \\
\hline Geometry & $1.27^{\star \star}$ & {$[1.25,1.28]$} \\
\hline \multicolumn{3}{|l|}{ Cognitive domain item (reasoning = ref) } \\
\hline Applying & $1.49^{\star \star}$ & {$[1.48,1.51]$} \\
\hline Knowing & $2.18^{\star \star}$ & {$[2.15,2.20]$} \\
\hline \multicolumn{3}{|l|}{ General language features } \\
\hline Total word count & $0.98^{\star \star}$ & {$[0.98,0.98]$} \\
\hline Number of different words & $0.98^{\star *}$ & {$[0.98,0.98]$} \\
\hline Total number of characters & $1.01^{\star \star}$ & {$[1.01,1.01]$} \\
\hline Number of characters without spaces & $0.99^{\star \star}$ & {$[0.99,0.99]$} \\
\hline Average syllables per Word & $1.47^{\star \star}$ & {$[1.45,1.49]$} \\
\hline Sentence count & $0.76^{\star \star}$ & {$[0.76,0.77]$} \\
\hline Averages sentence length in words & $0.95^{\star \star}$ & {$[0.95,0.95]$} \\
\hline Academic word use (AWL) & $0.99^{\star \star}$ & {$[0.98,0.99]$} \\
\hline \multicolumn{3}{|l|}{ Math language features } \\
\hline Quantitative language & $0.91^{\star \star}$ & {$[0.90,0.91]$} \\
\hline Spatial language & $0.89^{\star \star}$ & {$[0.88,0.89]$} \\
\hline Intercept & $0.04^{\star \star}$ & {$[0.03,0.04]$} \\
\hline N & 2721782 & \\
\hline
\end{tabular}

Note. $\mathrm{Cl}=$ confidence interval, ${ }^{*} p<.01,{ }^{* *} p<.001$. Country was coded as 58 dummy-variables, and included as covariate in our model (results not shown). 


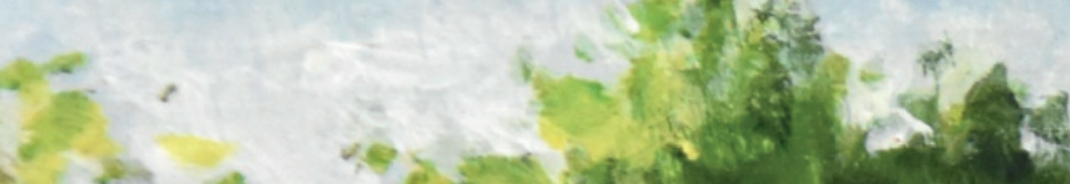

tes is un wat

$2 x-4 x^{2}$

\section{$2=$ \\ SW \\ $x_{2} \cos ^{2}$ \\ $8 x$ \\ $\approx$}

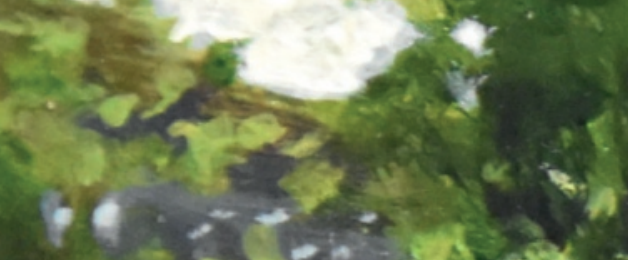

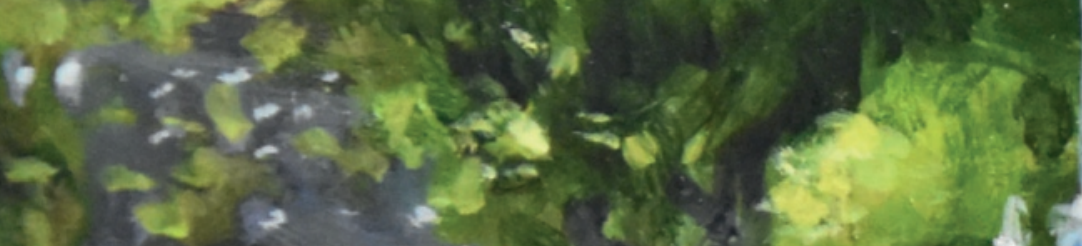

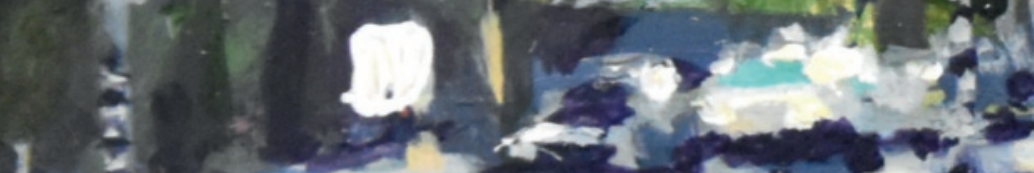

$1 \frac{2}{3}=2$

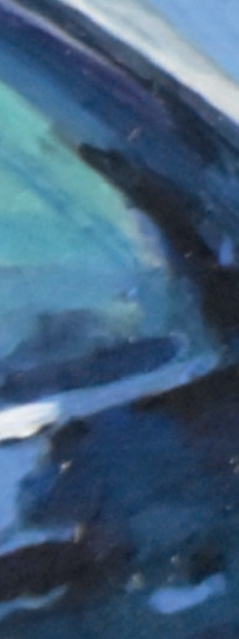


The Compensating Role of Personality in the Face of Background Disadvantage:

Predicting School Achievement getrom Childhood to Adolescence ${ }^{3}$ 


\begin{abstract}
The positive relation between socioeconomic status (SES) and educational outcomes is well-documented. In addition, previous research has shown that certain personality traits, such as Openness, predict educational outcomes. However, little is known about the possibility that personality predicts educational outcomes differently at various levels of SES. This study examines the interplay between students' SES and personality traits during childhood, in predicting educational performance (in terms of test performance and educational attainment) in middle adolescence, testing three hypotheses: (1) the independent effect hypothesis, i.e., personality traits predict educational attainment independent of students' SES, (2) the resource substitution hypothesis, i.e., personality traits are stronger predictors of educational attainment at lower levels of students' SES, and (3) the complementary effect hypothesis, i.e., personality traits are stronger predictors of educational attainment at higher levels of students' SES. We used a large representative longitudinal dataset from the Netherlands $(\mathrm{N}=7,409)$, controlling for background characteristics, including intelligence. With regard to students' test performance, results showed evidence for the independent effect hypothesis, showing that Emotional stability, Openness, and SES have independent positive relations with test performance. In contrast, with regard to students' educational attainment, we also find evidence for the resource substitution hypotheses: Openness is a stronger predictor of educational attainment at lower levels of students' SES. We conclude that for low-SES children, especially Openness may compensate for background disadvantage. We argue that the differential findings for test performance and educational attainment may suggest that the compensating role of personality may work through teachers' evaluations.
\end{abstract}




\subsection{Introduction}

A major discovery in the past decades for educational research, economy, and related social sciences, is that personality predicts important life outcomes, such as educational attainment, income and occupational status. In addition to IQ and socioeconomic status (SES), personality characteristics appeared to have substantial additional predictive power for future status attainment outcomes (Borghans, Duckworth, Heckman, \& Ter Weel, 2008; Heckman, 2006; Heckman \& Kautz, 2012; Moffitt et al., 2011; Roberts, Kuncel, Shiner, Caspi, \& Goldberg, 2007).

Whereas it is clear that both personality and other characteristics such as IQ and socioeconomic status (SES) are related to important life outcomes, little is known about the possibility that the relation between personality and SES is more complex. More specifically, personality and SES may interact, in a way that it depends on students' SES background, whether certain personality-traits, such as Openness, add to their future status attainment (Damian, Su, Shanahan, Trautwein, \& Roberts, 2015). For example, high levels of Openness predict educational achievement in primary school (Poropat, 2009), but this relation might vary with the student's SES.

The phase between childhood and adolescence (age 10 to 14) marks the important transition from primary school to (junior) high school, wherein children's academic trajectories are taking shape and academic prospects are determined (Akos, Lambie, Milsom, \& Gilbert, 2007). Therefore, it is of special importance to gain knowledge about the interplay between SES and personality related to academic performance in this developmental stage.

In this study, we investigate the relationship between SES background and the effects of personality traits, measured in a large and representative sample of students in their early adolescence in the south of the Netherlands $(\mathrm{N}=7,409)$, in predicting test performance and educational attainment three years later, in middle adolescence. Specifically, we examined whether personality traits show one of three patterns with relation to SES background and test performance and educational attainment, testing three hypotheses: (1) the independent effect hypothesis (i.e., personality traits predict educational attainment independent of students' SES) (Damian et al., 2015), (2) the resource substitution hypothesis (i.e., personality can compensate for effects of disadvantageous backgrounds: personality traits are stronger predictors of educational attainment at lower levels of students' SES) (Mirowsky \& Ross, 2003), and (3) the complementary effect hypothesis (i.e., personality traits are stronger predictors of educational attainment at higher levels of students' SES) (Merton, 1968). We are the first to examine these hypotheses by testing the moderating role of students' SES on the relationship between personality in childhood and educational outcomes in adolescence, using a longitudinal design with a large and representative sample of students from age 12 to age 15 . 


\section{SES and educational attainment}

Traditionally, social class or SES and the correlates with educational attainment was the domain of sociological literature (e.g., Bourdieu \& Passeron, 1977), showing that SES affects children's educational attainment (e.g., Duncan \& Brooks-Gunn, 1997). Metaanalyses show that SES at the student level is one of the strongest correlates of academic performance (White, 1982; Sirin, 2005). In addition, the size of the relationship between SES and educational attainment increases by each school level (Sirin, 2005), suggesting that the gap between low- and high-SES students widens through their lives. The relation between SES and educational achievement is mediated and reinforced by several factors, such as differences in cognitive development when children enter kindergarten (e.g., Hackman \& Farah, 2009), higher chance for low-SES students of being placed in low-resource schools compared to high-SES students (Lee \& Burkam, 2002), and lowSES students' higher chance to encounter lower educational expectations, both from their parents (e.g., Davis-Kean, 2005) and their teachers (e.g., Jussim, Eccles, \& Madon, 1996; Speybroeck et al., 2012). Moreover, poverty and scarcity tied to low-SES can have detrimental effects on cognitive functioning, affecting academic performance (Evans \& Schamberg, 2009; Mani, Mullainathan, Shafir, \& Zhao, 2013; Mullainathan \& Sharif, 2013). Specifically, it is shown that poverty has detrimental effect on early adolescents' school performance. More specifically, childhood poverty predicts lower mathematics and reading achievement through its associations with less cognitively stimulating and emotionally supportive home environments, and school behavior problems (Eamon, 2002). In addition, it is also suggested that there may be a mismatch between skills that children from disadvantaged circumstances develop in interaction with their home environments, and educational demands in school-environments, resulting in lower performance among children from low-SES environments (Ellis, Bianchi, Griskevicius, \& Frankenhuis, 2017; Rogoff et al., 2017; Sternberg, 2005; Valencia, 2010). In sum, SES is an important factor in predicting school performance in the phase from childhood to adolescence.

\section{Personality and educational attainment}

At the same time, psychological research focused on the predictive value of personality traits for educational attainment (e.g., Entwistle, 1972; Duckworth, Weir, Tsukayama, \& Kwok, 2012). In addition to IQ, a prominent predictor of educational attainment (Strenze, 2007), personality was also found to predict educational outcomes (Poropat, 2009). A dominant conceptualization of personality structure in the literature is the Five-Factor Model of personality (e.g., O'Connor, 2002), positing that Conscientiousness, Openness to experience (Openness), Extraversion, Agreeableness, and Emotional stability are the highest level of personality hierarchy, covering more narrow personality traits at lower levels of the hierarchy. Personality traits Agreeableness, Conscientiousness and Openness are found to correlate positively with academic performance during primary and secondary education (Poropat, 2009), and Conscientiousness has shown to have a unique contribution to grades at the end of high-school and during tertiary education (Noftle \& Robins, 2007; Poropat, 2009). In addition, meta-analysis showed that both Conscientiousness and 
Openness predict post-secondary academic achievement (O’Connor \& Paunonen, 2007). Personality may influence school performance through several pathways. First, the relation between personality and school performance can be mediated by students' school-related behavior. For example, self-management practices mediate the relationship between Conscientiousness and school performance (Gerhardt, Rode, \& Peterson, 2007). Second, students' personality traits may affect teachers' expectations, which in turn can affect school performance (Jussim et al., 1996). There has been little research that combines the focus on SES and personality, by testing interaction effects between SES and personality on educational outcomes.

\section{Independent effects}

The implicit standard approach towards the position of SES and personality in predicting education outcomes, is that both SES and personality predict educational attainment independently (the independent effect hypothesis), and interaction effects are seldom explicitly tested. However, Damian et al. (2015) tested the interaction between SES and personality during adolescence on status attainment in adulthood and found evidence for the independent effect hypothesis. Whereas this study also found some evidence for both the resource substitution hypothesis and the complementary effect hypothesis, these interaction effects disappeared after controlling for IQ. Therefore, Damian et al. (2015) concluded that their results show most robust evidence for the independent effect hypothesis.

\section{Resource substitution}

Yet, in the current literature, we also find evidence for the resource substitution hypothesis (Mirowsky \& Ross, 2003). This hypothesis refers to a pattern where resources have more beneficial effects when people have fewer alternative resources. Children from low-SES environments have less access to cultural capital than children from high-SES environments (e.g., De Graaf, De Graaf, \& Kraaykamp, 2000), and personality may serve as a resource that compensates for this deficiency. In the literature, we find some evidence for the resource substitution hypotheses with regard to outcomes in adulthood. For example, Shanahan, Bauldry, Roberts, Macmillan, and Russo (2014) showed that high-school students of less educated parents may benefit more from valued personality traits, such as Conscientiousness, in terms of education attainment during adulthood than high-school students with highly educated parents.

\section{Complementary effect}

Contrary to the resource substitution hypotheses, it may also be the case that personality traits are stronger predictors of educational attainment at higher SES-levels. The underlying mechanism would be that high-SES environments promote and facilitate the positive effects of specific personality traits more than low-SES environments, leading to a more positive relation between personality traits and educational outcomes for high-SES students than for low-SES students. For example, conscientiousness may be more encouraged and valued 
by high-SES parents, leading to reinforcement of behavior that is beneficial for educational outcomes (Damian et al., 2015). There is evidence for this complementary effect in a broad range of areas, for example the domain of educational research (high-SES young adults benefit more from school motivation than low-SES adults, Walberg, \& Tsai, 1983), and research on scientific careers (highly ranked scientist receive more reward for academic achievements than their lower ranked colleagues, Merton, 1968).

\section{Late childhood and middle adolescence}

Although the theoretical and practical importance of testing our hypotheses, current literature has not examined the complexity of the relation between SES and personality in relation to educational outcomes from childhood to adolescence. In the Netherlands, as in many western countries, the timespan from late childhood (age 12) to middle adolescence (age 15) marks the important transition from primary to secondary education. Achievement during these years strongly determines students' educational careers, because of the tracked system that assigns students into different educational programs based on their performance. This tracking determines and limits the possibilities in the transition from secondary to tertiary education. For example, tracking in the highest level gives access to universities, and tracking in a lower track only gives access to vocational education. Although the Dutch system provides possibilities to 'repair' educational pathways at a higher age, the level of track assigned to between age 12 and 15 is highly predictive for future educational attainment (OECD, 2016). Hence, the role of personality and SES in educational attainment during age 12 to age 15 may have long-term consequences and are therefore important to examine and understand. A better understanding of these relations, and of the possibility that SES and personality interact, may open up the possibility to intervene in this early age phase in order to diminish the SES-gap in educational performance (Heckman \& Kautz, 2012).

\section{Present study}

In this study, we investigate the predictive value of personality traits during childhood for educational attainment in adolescence, as well as the moderating role of students' SES background. As stated earlier, we will test the following three hypothesis: (1) the independent effect hypothesis (i.e., personality traits predict educational attainment independent of students' SES), (2) the resource substitution hypothesis (i.e., personality can compensate for effects of disadvantageous backgrounds: personality traits are stronger predictors of educational attainment at lower levels of students' SES), and (3) the complementary effect hypothesis (i.e., personality traits are stronger predictors of educational attainment at higher levels of students' SES). Our research adds to the current literature, by testing our hypothesis in a longitudinal design with a large and representative sample. Because of the longitudinal design, this dataset provides a unique opportunity to examine the predictive value of personality, SES, and the interaction between personality and SES during childhood for educational outcomes in adolescence. These data allow us to control for important background variables, such as cognitive ability. 


\subsection{Method}

\section{Data}

This study was initially part of an exploratory research project commissioned by the Dutch Ministry of Education, Culture and Science. Original research questions, inclusion criteria, and constructs of this study were determined before conducting data analyses. In this section, we report how we determined our sample size, data exclusions, and all measures in the study, as well as our statistical approach.

We applied data from the Educational Monitor Limburg (OML, Onderwijs Monitor Limburg in Dutch), a longitudinal study in the south of the Netherlands, developed by Maastricht University in cooperation with the Province of Limburg, and primary, secondary and tertiary education institutes in the south of the Netherlands. Every two years, a representative sample of students from grade 6 participate in the OML. In our study, we apply data from the cohorts 2009 and 2011. Three years later, in grade 9, we recontacted all participants via their schools. Among schools for secondary education who participated in the follow-up ( $91 \%$ for cohort $2009,82 \%$ for cohort 2011), the response rate was 82 percent for cohort 2009, and 79 percent for cohort 2011, resulting in a sample with 7,409 participants who participated in grade 6 and grade 9 . We present attrition analyses in the results section.

\section{Measures}

Personality traits. The OML 2009 and 2011 (grade 6) included 22 items based on the IPIP NEO 120 (Johnson, 2014) from which five Big-Five personality traits were scored. Conscientiousness and Extraversion were captured with five items, and Openness, Agreeableness and Emotional Stability with four items. These items consisted of statements regarding their personality or behavior, for example 'I like order and regularity'. For each item, participant rated how well the item described them on a five-point scale $(1=$ not at all agree, 5 = strongly agree). We applied McDonald's Omega to analyze the reliability of the scales (Trizano-Hermosilla, \& Alvarado, 2016), for Openness $(\omega=.60)$, Conscientiousness $(\omega=.65)$, Extraversion $(\omega=.60)$, Agreeableness $(\omega=.66)$, and Emotional Stability $(\omega=.75)$. Because of the clear interpretation of sum scores, we applied the sum score by factor method and computed average scores for each trait (Tabachnick \& Fidell, 2001) for descriptive statistics.

Cognitive ability. In grade 6, students have completed an IQ-test, based on 44 items from Raven's Standard Progressive Matrices (Raven \& Court, 1998). We applied a two-parameter logistic (2PL) item response model (Kean \& Reilly, 2014) to compute the IQ score for each student.

SES. We constructed a measure for SES based on parental educational attainment consisting of 8 ISCED-levels (UNESCO Institute for Statistics, 2012), and standardized them prior to the analyses. 
Demographic measures. We included three demographic measures in all the analyses, because their well-documented relationship between these variables and educational attainment: age, gender and ethnicity. We standardized age prior to analyzes. Ethnicity was coded on a 3 -point scale $(1=$ non-immigrant, 2 = western immigrant, and $3=$ nonwestern immigrant), and we constructed one dummy-variable out of this scale (1 = migrant, $0=$ non-migrant). Gender was coded as male $=0$, female $=1$.

Educational attainment. In Grade 9, students are enrolled in secondary education and assigned to an educational level. In the original data, educational level was measured with an 8-point scale, varying from preparatory vocational education (1) to pre-university education (8). In the original coding, scores 1 through 5 referred to four levels of preparatory vocational education, score 6 referred to HAVO (high school preparing for higher vocational education), score 7 referred to pre-university education without classical languages, and score 8 referred to pre-university education with classical languages. Because about $25 \%$ of all students attend HAVO, about $50 \%$ of all students attend some form of preparatory vocational education, and only a small minority attends pre-university education with classical languages (Dutch Inspectorate of Education, 2018), this scale was not well balanced. To address this issue, we recoded this scale as follows. Score 1, 2 and 3 kept the same coding. Score 4 and 5 became ' 4 ' (middle preparatory vocational education), score 6 became ' 5 ' (higher preparatory vocational education), and score 7 and 8 became ' 6 ' (pre-university education). In this new scale, the mean educational level in Grade 9 was 4.35 with a standard deviation of 1.57 , indicating that the average individual in Grade 9 attends middle preparatory vocational education. In addition, after recoding, the distance between each scale unit translates into one educational level. These levels determine the eligibility for tertiary education. For example, higher preparatory vocational education gives access to higher vocational education, and only pre-university education gives access to universities. Currently, there is a societal debate in the Netherlands about the appraisal of these different tracks. One question is whether we should classify these tracks as 'lower' and 'higher', or whether it would be more accurate to classify these tracks from 'practical oriented' to 'theoretical oriented'. While we are aware of this debate, in this study we apply educational track in Grade 9 (level 1 to 6 ) in this study as a scale from low to high.

Math test. In grade 9, participants filled in a Dutch standardized math test, with 92 math items. We applied a two-parameter logistic (2PL) item response model (Kean \& Reilly, 2014) to compute a math score for each student.

Language test. Respondents filled in a Dutch standardized language test that measures their language skills, consisting of 99 items. With a two-parameter logistic (2PL) item response model (Kean \& Reilly, 2014), we computed a language score for each respondent. 


\section{Data cleaning}

No participants were excluded from the data-analyses.

\section{Data analyses}

We applied educational level, performance on a standardized math test, and performance on a standardized language test (all measured in grade 9) as outcome variables. Our main analyses are a series of five linear regression models (one model per personality trait) for each outcome. In each model, one of the three outcome variables were included as dependent variable, and personality trait, SES and the interaction between personality trait and SES were included as predictors. Each model included the controls gender, migrant status, age, intelligence, and the four other big-five traits. All analyses were conducted with STATA 13. To account for random missing data, we used Full-information Maximum Likelihood (FIML). Applying FIML minimizes potential biases caused by random missing data in an efficient way (Enders \& Bandalos, 2001).

TABLE 1 Descriptive Statistics and Correlations between All Variables

\begin{tabular}{|c|c|c|c|c|c|c|c|c|c|c|c|c|c|c|c|}
\hline No. & Variable & $M / \%$ & SD & 1 & 2 & 3 & 4 & 5 & 6 & 7 & 8 & 9 & 10 & 11 & 12 \\
\hline 1 & Gender & $51 \%$ & - & - & & & & & & & & & & & \\
\hline 2 & Migrant & $13 \%$ & - & .00 & - & & & & & & & & & & \\
\hline 3 & Age (G9) & 15.28 & 0.52 & -.08 & .06 & - & & & & & & & & & \\
\hline 4 & SES & 0.00 & 1.00 & -.03 & -.01 & -.19 & - & & & & & & & & \\
\hline 5 & Intelligence & 0.03 & 0.88 & .02 & -.01 & -.13 & .20 & - & & & & & & & \\
\hline 6 & Openness & 3.28 & 0.69 & -.10 & -.01 & -.09 & .17 & .20 & - & & & & & & \\
\hline 7 & Conscientiousness & 3.12 & 0.67 & .06 & .02 & -.02 & .05 & .09 & .18 & - & & & & & \\
\hline 8 & Extraversion & 3.25 & 0.68 & .00 & -.01 & .03 & .01 & -.03 & .19 & .02 & - & & & & \\
\hline 9 & Agreeableness & 4.08 & 0.53 & .25 & -.01 & -.04 & .05 & .03 & .26 & .31 & .26 & - & & & \\
\hline 10 & Emotional stability & 3.45 & 0.81 & -.07 & -.03 & -.03 & .06 & .06 & .07 & .30 & .17 & .18 & - & & \\
\hline 11 & Educational Attainment (Gو) & 4.35 & 1.57 & .02 & -.04 & -.32 & .40 & .42 & .32 & .13 & -.02 & .11 & .13 & - & \\
\hline 12 & Math performance (G9) & 0.06 & 0.89 & -.04 & -.03 & -.24 & .26 & .35 & .23 & .07 & -.05 & .04 & .06 & .60 & - \\
\hline 13 & Language performance (Gg) & 0.10 & 0.90 & .12 & -.03 & -.24 & .21 & .28 & .21 & .07 & -.06 & .08 & .05 & .51 & .56 \\
\hline
\end{tabular}

Note. Gender and Migrant are dummy codes ( $1=$ female, $0=$ male; $1=$ migrant, $0=$ non-migrant). Parental SES is a standardized score, all other variables are raw scores. Bold font indicates that the $95 \%$ confidence interval did not include zero. $N=7,409$

\subsection{Results}

Table 1 presents descriptive statistics and correlations between variables included in our analyses. These results indicate a weak positive correlation between SES and the personality traits Openness, Conscientiousness, Agreeableness, and Emotional Stability. 
Table 2 presents attrition analyses. Of the approximately 9,000 participants available in grade 6 , about 7,500 responded in grade 9 . These results of these attrition analyses show that the students who stayed in the study, as opposed to those who did not participate in grade 9, had a slightly higher parental SES $(r=.15)$, were more intelligent $(r=.11)$, and had slightly higher levels of Agreeableness $(r=.02)$ and Emotional Stability $(r=.09)$. These results did not indicate differences between respondents who stayed in the study and respondents who dropped out with regard to Openness, Conscientiousness and Extraversion. In addition, the gender distribution was different for students who participated twice ( $51 \%$ female) compared to respondents who did not participate in grade 9 ( $44 \%$ female). One reason for attrition was that some students were not present during the data-collection in grade 9, mostly due to illness or because of moving house to another region or country. However, a more important reason for drop out was the transition to special needs education after grade 6 . In the Netherlands, about $6 \%$ of all students attend schools for special educational needs from grade 7 onwards (Dutch Inspectorate of Education, 2018). Therefore, the differences we found in these attrition analyses can probably largely be explained by the drop out from this study from students with learning difficulties (such as low IQ or behavioral problems). Therefore, the data we apply in this study can be considered as a representative sample of students in the Netherlands, but only for students who attend regular schools.

\section{Prospective effects of personality traits on test performance}

Math performance. We tested the relation between personality traits, SES, and students' math test performance with separate analyses for each personality trait (i.e., Openness, Conscientiousness, Extraversion, Agreeableness, Emotional Stability). The models include math test performance as dependent variable, one personality trait, SES, and personality trait $\times$ SES as predictors, and gender, migrant-status, age, intelligence, and the four other big-five personality traits as covariates. Table 3 presents the results for each personality trait with performance on math tests as dependent variable. The personality traits Openness and Emotional Stability show a significant positive relation with math performance. In contrast, Extraversion shows a negative relation with math performance. In addition, students' SES and intelligence show a significant positive relation with math performance. Importantly, results show no interaction between personality traits and SES. These results support the independent effects hypotheses: SES and personality appear to have independent relations with math performance. 
TABLE 2 Attrition Analyses

\begin{tabular}{llll}
\hline Variable & Mean Difference & $95 \% \mathrm{Cl}$ & $\mathrm{r}$ \\
\hline SES & -.47 & {$[-.41,-.53]$} & -.15 \\
Intelligence & -.25 & {$[-.20,-.30]$} & -.11 \\
Openness & -.06 & {$[.00,-.11]$} & -.03 \\
Conscientiousness & -.01 & {$[.05,-.06]$} & -.01 \\
Extraversion & -.02 & {$[.03,-.08]$} & -.01 \\
Agreeableness & -.06 & {$[-.01,-.12]$} & -.02 \\
Emo. stability & -.22 & {$[-. .17,-.28]$} & -.09 \\
\hline
\end{tabular}

Note. Negative relations mean higher scores for the respondents who stayed in the study. All variables were standardized prior to the analyses. $\mathrm{Cl}=$ confidence interval; SES = socioeconomic status.

TABLE 3 Linear Regression Models Predicting Performance on a Standardized Math Test in Grade 9 (Separate Analyses for Each Personality Trait)

\begin{tabular}{|c|c|c|c|c|c|c|c|c|c|c|}
\hline & \multicolumn{2}{|c|}{ Openness } & \multicolumn{2}{|c|}{ Conscientiousness } & \multicolumn{2}{|c|}{ Extraversion } & \multicolumn{2}{|c|}{ Agreeableness } & \multicolumn{2}{|c|}{ Emotional stability } \\
\hline & $\beta$ & $95 \% \mathrm{Cl}$ & $\beta$ & $95 \% \mathrm{Cl}$ & $\beta$ & $95 \% \mathrm{Cl}$ & $\beta$ & $95 \% \mathrm{Cl}$ & $\beta$ & $95 \% \mathrm{Cl}$ \\
\hline Personality trait & 0.17 & {$[0.12,0.21]$} & 0.01 & {$[-0.03,0.05]$} & -0.04 & {$[-0.16,-0.09]$} & 0.01 & {$[-0.03,0.05]$} & 0.04 & {$[0.01,0.08]$} \\
\hline SES & 0.13 & {$[0.10,0.15]$} & 0.14 & {$[0.11,0.16]$} & 0.13 & {$[0.11,0.16]$} & 0.14 & {$[0.11,0.16]$} & 0.13 & {$[0.11,0.16]$} \\
\hline Personality $\times$ SES & 0.00 & {$[-0.02,0.03]$} & 0.00 & {$[-0.02,0.03]$} & 0.01 & {$[-0.02,0.03]$} & -0.00 & {$[-0.03,0.02]$} & -0.00 & {$[-0.03,0.02]$} \\
\hline \multicolumn{11}{|l|}{ Covariates } \\
\hline Female & -0.04 & {$[-0.09,0.01]$} & -0.07 & {$[-0.11,-0.02]$} & -0.07 & {$[-0.12,-0.02]$} & -0.07 & {$[-0.12,-0.02]$} & -0.06 & {$[-0.11,-0.02]$} \\
\hline Migrant & -0.02 & {$[-0.08,0.04]$} & -0.01 & {$[-0.08,0.05]$} & -0.01 & {$[-0.07,0.05]$} & -0.01 & {$[-0.08,0.05]$} & -0.01 & {$[-0.07,0.05]$} \\
\hline Age & -0.13 & {$[-0.16,-0.11]$} & -0.13 & {$[-0.16,-0.11]$} & -0.13 & {$[-0.15,-0.11]$} & -0.14 & {$[-0.16,-0.11]$} & -0.14 & {$[-0.16,-0.11]$} \\
\hline Intelligence & 0.26 & {$[0.23,0.29]$} & 0.28 & {$[0.25,0.31]$} & 0.27 & {$[0.25,0.30]$} & 0.28 & {$[0.25,0.31]$} & 0.28 & {$[0.25,0.31]$} \\
\hline Openness & . & & 0.12 & {$[0.10,0.15]$} & 0.14 & {$[0.11,0.16]$} & 0.12 & {$[0.10,0.15]$} & 0.13 & {$[0.10,0.15]$} \\
\hline Conscientiousness & -0.01 & {$[-0.03,0.02]$} & . & & -0.00 & {$[-0.03,0.03]$} & 0.01 & {$[-0.02,0.04]$} & 0.00 & {$[-0.03,0.03]$} \\
\hline Extraversion & -0.09 & {$[-0.12,-0.07]$} & -0.08 & {$[-0.10,-0.05]$} & . & & -0.08 & {$[-0.10,-0.05]$} & -0.08 & {$[-0.12,-0.05]$} \\
\hline Agreeableness & -0.00 & {$[-0.03,0.03]$} & 0.01 & {$[-0.02,0.04]$} & 0.04 & {$[0.01,0.07]$} & . & . & 0.01 & {$[-0.01,0.04]$} \\
\hline Emo. stability & 0.04 & {$[0.02,0.07]$} & 0.04 & {$[0.01,0.06]$} & 0.05 & {$[0.02,0.07]$} & 0.04 & {$[0.01,0.06]$} & . & . \\
\hline Constant & 0.07 & {$[0.04,0.11]$} & 0.08 & {$[0.05,0.12]$} & 0.09 & {$[0.05,0.12]$} & 0.08 & {$[0.05,0.12]$} & 0.08 & {$[0.05,0.11]$} \\
\hline $\mathrm{R}^{2}$ adj & 0.21 & & 0.21 & & 0.21 & & 0.21 & & 0.21 & \\
\hline
\end{tabular}

Note. Model controls for gender, migrant-status, age, IQ and IQ-square. Standardized regressions coefficients $\beta$. Math performance, Personality traits, SES, and IQ were standardized before analyzing. Level (Gg) yields six levels. Bold font indicates that the $95 \%$ confidence interval did not include zero. R2 adj = adjusted for number of predictors. $N=7,409$.

Language performance. Next, we applied the same models and used performance on a standardized language test as dependent variable (Table 4). Again, Openness and Emotional stability show a positive relation with performance on a language test. In addition, Extraversion appeared to predict lower performance on the language test. Students' SES has a positive relation with language performance. Interestingly, we found no interaction between personality and SES, indicating support for the independent effect hypothesis. 
TABLE 4 Linear Regression Models Predicting Performance on a Standardized Language Test in Grade 9 (Separate Analyses for Each Personality Trait)

\begin{tabular}{|c|c|c|c|c|c|c|c|c|c|c|}
\hline & \multicolumn{2}{|c|}{ Openness } & \multicolumn{2}{|c|}{ Conscientiousness } & \multicolumn{2}{|c|}{ Extraversion } & \multicolumn{2}{|c|}{ Agreeableness } & \multicolumn{2}{|c|}{ Emotional stability } \\
\hline & $\beta$ & $95 \% \mathrm{Cl}$ & $\beta$ & $95 \% \mathrm{Cl}$ & $\beta$ & $95 \% \mathrm{Cl}$ & $\beta$ & $95 \% \mathrm{Cl}$ & $\beta$ & $95 \% \mathrm{Cl}$ \\
\hline Personality trait & 0.20 & {$[0.15,0.24]$} & -0.01 & {$[-0.04,0.03]$} & -0.12 & {$[-0.16,-0.09]$} & 0.01 & {$[-0.03,0.05]$} & 0.04 & {$[0.01,0.07]$} \\
\hline SES & 0.09 & {$[0.07,0.12]$} & 0.10 & {$[0.08,0.13]$} & 0.10 & {$[0.08,0.13]$} & 0.10 & {$[0.08,0.13]$} & 0.10 & {$[0.08,0.13]$} \\
\hline Personality $\times$ SES & 0.02 & {$[-0.01,0.04]$} & -0.01 & {$[-0.03,0.02]$} & -0.00 & {$[-0.02,0.03]$} & -0.01 & {$[-0.04,0.02]$} & -0.01 & {$[-0.04,0.01]$} \\
\hline
\end{tabular}

Covariates

\begin{tabular}{|c|c|c|c|c|c|c|c|c|c|c|}
\hline Female & 0.24 & {$[0.19,0.29]$} & 0.21 & {$[0.17,0.26]$} & 0.21 & {$[0.16,0.26]$} & -0.21 & {$[0.16,0.26]$} & 0.22 & {$[0.17,0.27]$} \\
\hline Migrant & -0.02 & {$[-0.09,0.04]$} & -0.01 & {$[-0.08,0.05]$} & -0.01 & {$[-0.08,0.05]$} & -0.02 & {$[-0.08,0.05]$} & $-0 .-1$ & {$[-0.08,0.05]$} \\
\hline Age & -0.15 & {$[-0.17,-0.12]$} & -0.15 & {$[-0.17,-0.12]$} & -0.15 & {$[-0.17,-0.12]$} & -0.15 & {$[-0.17,-0.12]$} & -0.15 & {$[-0.17,-0.12]$} \\
\hline Intelligence & 0.19 & {$[0.16,0.22]$} & 0.21 & {$[0.18,0.24]$} & 0.21 & {$[0.18,0.24]$} & 0.21 & {$[0.18,0.24]$} & 0.21 & {$[0.18,0.24]$} \\
\hline Openness & & & 0.14 & {$[0.11,0.17]$} & 0.15 & {$[0.13,0.18]$} & 0.14 & {$[0.11,0.17]$} & 0.14 & {$[0.12,0.17]$} \\
\hline Conscientiousness & -0.01 & {$[-0.04,0.01]$} & & & -0.01 & {$[-0.04,0.02]$} & -0.00 & {$[-0.03,0.03]$} & -0.01 & {$[-0.04,0.02]$} \\
\hline Extraversion & -0.10 & {$[-0.13,-0.07]$} & -0.08 & {$[-0.11,-0.05]$} & & & -0.08 & {$[-0.11 .-0.05]$} & -0.08 & {$[-0.11,-0.05]$} \\
\hline Agreeableness & -0.01 & {$[-0.04,0.02]$} & 0.02 & {$[-0.02,0.05]$} & 0.04 & {$[0.00,0.07]$} & & & 0.01 & {$[-0.02,0.04]$} \\
\hline Emo. stability & 0.04 & {$[0.01,0.06]$} & 0.03 & {$[0.00,0.06]$} & 0.04 & {$[0.02,0.07]$} & 0.03 & {$[0.01,0.06]$} & & \\
\hline Constant & -0.04 & {$[-0.07,-0.00]$} & -0.02 & {$[-0.05,0.01]$} & -0.02 & {$[-0.05,0.01]$} & -0.02 & {$[-0.05,0.02]$} & -0.02 & {$[-0.05,0.02]$} \\
\hline 2 adj & 0.16 & & 0.16 & & 0.16 & & 0.16 & & 0.16 & \\
\hline
\end{tabular}

Note. Model controls for gender, migrant-status, age, IQ and IQ-square. Standardized regressions coefficients $\beta$. Math performance, Personality traits, SES, and IQ were standardized before analyzing. Level (Gg) yields six levels. Bold font indicates that the $95 \%$ confidence interval did not include zero. R2 adj $=$ adjusted for number of predictors. $N=7,409$.

\section{Prospective effects of personality traits on educational attainment}

We applied the same models, and used educational attainment in grade 9 as outcome variable. Table 5 presents the results for each personality trait (i.e., Openness, Conscientiousness, Extraversion, Agreeableness, Emotional Stability). Openness and Emotional stability show a positive relation with educational attainment. In contrast, Extraversion shows a negative relation with educational attainment. In addition, main effects of students' SES are positively related to educational attainment. In order to interpret the main effects of personality traits and SES, we present these results translated into natural metrics (see Table 6). For example, moving up (or down) two standard deviations in Openness in Grade 6, is related to 1.2 educational levels higher (or lower) on a 6-point scale in Grade 9. In addition, moving two standard deviations in SES, which has the largest main effect in our model, is related to a chance of 1.88 educational levels higher or lower on a 6-point scale. 
TABLE 5 Linear Regression Models Predicting Educational attainment in Grade 9 (Separate Analyses for Each Personality Trait)

\begin{tabular}{|c|c|c|c|c|c|c|c|c|c|c|}
\hline & \multicolumn{2}{|c|}{ Openness } & \multicolumn{2}{|c|}{ Conscientiousness } & \multicolumn{2}{|c|}{ Extraversion } & \multicolumn{2}{|c|}{ Agreeableness } & \multicolumn{2}{|c|}{ Emotional stability } \\
\hline & $\beta$ & $95 \% \mathrm{Cl}$ & $\beta$ & $95 \% \mathrm{Cl}$ & $\beta$ & $95 \% \mathrm{Cl}$ & $\beta$ & $95 \% \mathrm{Cl}$ & $\beta$ & $95 \% \mathrm{Cl}$ \\
\hline Personality trait & 0.64 & {$[0.57,0.71]$} & 0.05 & {$[-0.00,0.11]$} & -0.22 & {$[-0.28,-0.17]$} & 0.01 & {$[-0.05,0.07]$} & 0.18 & {$[0.13,0.23]$} \\
\hline SES & 0.52 & {$[0.48,0.56]$} & 0.56 & {$[0.52,0.59]$} & 0.55 & {$[0.52,0.59]$} & 0.56 & {$[0.52,0.59]$} & 0.56 & {$[0.52,0.59]$} \\
\hline Personality $\times$ SES & -0.05 & {$[-0.10,-0.01]$} & -0.03 & {$[-0.06,0.01]$} & 0.01 & {$[-0.03,0.05]$} & -0.06 & {$[-0.10,-0.02]$} & -0.03 & {$[-0.07,0.01]$} \\
\hline \multicolumn{11}{|l|}{ Covariates } \\
\hline Female & 0.22 & {$[0.14,0.30]$} & 0.09 & {$[0.02,0.16]$} & 0.09 & {$[0.01,0.16]$} & 0.10 & {$[0.02,0.18]$} & 0.11 & {$[0.04,0.18]$} \\
\hline Migrant & -0.12 & {$[-0.23,-0.02]$} & -0.12 & {$[-0.22 .-0.02]$} & -0.11 & {$[-0.22,-0.01]$} & -0.12 & {$[-0.22,-0.02]$} & -0.11 & {$[-0.21,-0.01]$} \\
\hline Age & -0.39 & {$[-0.42,-0.35]$} & -0.41 & {$[-0.44,-0.37]$} & -0.40 & {$[-0.44,-0.37]$} & -0.41 & {$[-0.44,-0.37]$} & -0.41 & {$[-0.44,-0.37]$} \\
\hline Intelligence & 0.60 & {$[0.55,0.65]$} & 0.68 & {$[0.64,0.72]$} & 0.67 & {$[0.62,0.72]$} & 0.68 & {$[0.64,0.73]$} & 0.68 & {$[0.64,0.73]$} \\
\hline Openness & & & 0.42 & {$[0.38,0.46]$} & 0.44 & {$[0.40,0.49]$} & 0.42 & {$[0.39,0.47]$} & 0.43 & {$[0.39,0.47]$} \\
\hline Conscientiousness & -0.01 & {$[-0.05,0.03]$} & & & 0.03 & {$[-0.01,0.07]$} & 0.05 & {$[0.01,0.10]$} & 0.03 & {$[-0.01,0.07]$} \\
\hline Extraversion & -0.20 & {$[-0.25,-0.16]$} & -0.13 & {$[-0.17,-0.09]$} & & & -0.13 & {$[-0,17,-0.09]$} & -0.15 & {$[-0.19,-0.11]$} \\
\hline Agreeableness & -0.06 & {$[-0.11,-0.01]$} & 0.03 & {$[-0.02,0.07]$} & 0.08 & {$[0.03,0.12]$} & & & 0.03 & {$[-0.01,0.07]$} \\
\hline Emo. stability & 0.17 & {$[0.12,0.21]$} & 0.14 & {$[0.10,0.18]$} & 0.17 & {$[0.12,0.21]$} & 0.15 & {$[0.11,0.19]$} & & \\
\hline Constant & 5.04 & {$[4.99,5.10]$} & 5.11 & {$[5.05,5.16]$} & 5.111 & {$[5.06,5.16]$} & 5.10 & {$[5.05,5.16]$} & 5.10 & {$[5.04,5.15]$} \\
\hline $\mathrm{R}^{2}$ adj & 0.36 & & 0.36 & & 0.36 & & 0.36 & & 0.36 & \\
\hline
\end{tabular}

Note. Model controls for gender, migrant-status, age, IQ, and IQ-square. Standardized regressions coefficients $\beta$. Math performance, Personality traits, SES, and IQ were standardized before analyzing. Level (Gg) yields six levels. Bold font indicates that the $95 \%$ confidence interval did not include zero. R2 adj = adjusted for number of predictors. $N=7,409$.

TABLE 6 Main Effect Estimates of Each Predictor in Natural Metrics

\begin{tabular}{llcc}
\hline & \multicolumn{3}{c}{ Educational attainment (6 levels) } \\
\cline { 2 - 4 } & $\mathbf{1 ~ S D}$ & $\mathbf{- 1}$ to $\mathbf{1}$ SD & $\mathbf{- 2}$ to $\mathbf{2}$ SD \\
\hline Openness & 0.64 level & 1.28 level & 2.56 level \\
Conscientiousness & - & - & - \\
Extraversion & -0.22 level & -0.44 level & -0.88 level \\
Agreeableness & - & - & - \\
Emotional stability & 0.18 level & 0.36 level & 0.72 level \\
SES & 0.55 level & 1.10 level & 2.20 level \\
\hline
\end{tabular}

Note. Educational attainment (6 levels) in Grade 9, varying from preparatory vocational education (1) to pre-university education (6). The main effect of SES varied between 0.52 to 0.56 across the five personality regressions. Therefore, we present the average main effect of SES across the five regressions within each outcome (i.e., 0.55)

Importantly, results show a statistically significant interaction between Openness and SES. This significant interaction-effect supports the resource substitution hypothesis: Openness is a stronger predictor of educational attainment at lower levels of students' SES. For example, for students at lowest level on the SES-scale, 2 SD higher in Openness in Grade 6 predicts a 2.56 elevation in educational level in Grade 9. The interaction indicates 
that each SD higher on the SES-scale is related to a decrease of the relation between Openness and educational level of 0.05 educational level. This compensating effect can be considered as substantial, and according to these data, it could generate a complete compensation of the disadvantage tied to a low-SES background (e.g., a difference of 2 SD in SES is related to 2.20 levels difference in educational level).

Results also show an interaction between SES and Agreeableness, but this interaction does not support one of our three hypotheses. This interaction indicates that for low-SES students Agreeableness does not predict educational attainment, whereas for high SES students, higher levels of Agreeableness predict a lower educational attainment.

Personality trait Emotional stability shows a pattern that is in line with the independenteffect hypotheses: this traits is positively related to educational attainment, and this relation does not vary by students' socioeconomic background.

\subsection{Discussion}

Previous research has shown that both children's personality and socioeconomic background are predictors of educational attainment during adolescence, implicitly suggesting that these characteristics have independent effects. In this study, we investigated whether the relation between personality traits and socioeconomic status is more complex, testing three hypotheses: (1) the independent effect hypothesis (i.e., personality traits predict educational attainment independent of students' SES), (2) the resource substitution hypothesis (i.e., personality can compensate for effects of disadvantageous backgrounds: personality traits are stronger predictors of educational attainment at lower levels of students' SES), and (3) the complementary effect hypothesis (i.e., personality traits are stronger predictors of educational attainment at higher levels of students' SES). We examined these hypotheses in a longitudinal dataset with personality- and SES-measures in late childhood (Grade 6) and educational outcomes in middle adolescence (Grade 9).

Consistent with previous research, results showed positive main effects for personality traits Openness and Emotional stability during late childhood on educational outcomes (in terms of test performance and educational attainment) three years later. In addition, our results showed the well-documented positive relation between SES and educational outcomes (i.e., test performance and educational attainment). With regard to test performance (i.e., math and language), results show evidence for the independent effects hypotheses: both SES and personality traits appeared to predict test performance independently. Importantly, with regard to educational attainment, we found evidence for the resource substitution hypothesis for the personality trait Openness. At lower levels of students' SES, this personality trait is more predictive for educational attainment than at higher levels of students' SES, also after controlling for intelligence. Surprisingly, the personality trait Conscientiousness shows no signs of contribution to test performance nor 
educational attainment, which is intriguing because this aspect of personality is typically found to be a reliable predictor of attained status (Borghans et al., 2008). However, this finding may reflect a limited content validity of the instrument.

We also found that SES is weak and positively correlated with personality traits that predict school performance (i.e., Openness and Emotional Stability), indicating that lowSES students have a slightly lower change of displaying these personality traits, enhancing the educational gap between low-and high SES students.

\section{Personality and educational outcomes}

How can personality traits affect test performance and educational attainment, given one's intelligence? Literature, assuming causal relationships between personality traits and school performance, suggests that both school-related behavior and teachers' expectations may play a role. First, children who have, for example, higher levels of Openness, are probably more interested in academic matters and therefore, and may put more effort in school-related tasks, enhancing their ability level and educational attainment (Hazrati-Viari, Rad, \& Torabi, 2012). Second, teachers may notice higher levels of students' Openness, elevating their expectations which in turn may contribute to educational attainment (Jussim et al., 1996). The finding that Openness and Emotional Stability predict both tests performance (which is an objective ability measure) and educational attainment (which is influenced by teachers' evaluations), indicates that the positive relation between Openness in late childhood and school performance in middle adolescence, may work through both beneficial school behavior and teachers' expectations.

Openness is generally considered as a personality trait involving six correlating facets, including intellectual curiosity, preference for nontraditional values, the tendency to fantasize, an aesthetic sensitivity, awareness of one's emotions, and preference for novelty (McCrae \& Costa, 1997), and has been found in many studies to have a positive relationship with school performance (e.g., Poropat, 2009). Our results evoke the question why Openness may work stronger, or 'compensate disadvantage', at lower levels of students' SES. One possibility is that the compensatory effects result from the absence of alternative resources for low-SES students. Probably, students from high-SES environments can rely on other resources (i.e., cultural capital) than their personality in reaching high educational attainment, whereas low-SES students have less access to these alternative resources. Therefore, Openness may be more predictive for educational attainment among low-SES students than among high-SES students. In addition, the finding that Openness can compensate disadvantage with regard to educational attainment, which is highly determined by teachers' evaluations, but not with regard to standardized test performance, indicates that the compensating role of Openness may work through teachers' evaluations. Although speculative, we conclude that among low-SES students, who are more likely to meet low teacher expectations (Jussim et al., 1996; Speybroeck et al., 2012), displaying Openness may be a powerful way to influence these expectations. 
Interestingly, Damian et al. (2015) showed evidence for the independent-effect hypotheses regarding Openness in adolescence and status attainment during adulthood. How can we explain that we find, in contrast, evidence for the resource-substitution hypotheses from childhood to adolescence regarding Openness? We think one explanation can be found in the timing of important transitions in educational careers. Personality may have stronger compensatory effects during important transitions in early education than in later, less determining stages of students' development. Whereas in adolescence, students are already sorted out to a certain degree towards their final educational level, in late childhood, there is a wider range of possible future outcomes (Woessmann, 2009). Therefore, Openness can have compensatory effects from childhood to adolescence, and at the same time only have independent effects from adolescence to adulthood.

These findings, combining psychological and sociological constructs, add to literature about personality and resilience. Resilient adolescents, i.e., individuals who succeed in age-salient developmental tasks despite significant adversity throughout childhood and adolescence, differ from less resilient adolescents on personality traits (Shiner, \& Masten, 2012). During childhood, they show higher childhood Openness, Conscientiousness, Agreeableness, and Emotional Stability. In other words, these personality traits during childhood predict adult resilience. Our finding may suggest that in particular Openness may have additional predictive value in the development of resilience among low-SES children.

\section{Development of personality}

Importantly, because of the observational nature of research on relationships between personality and school performance, it is still a possibility that these relationships are not causal. An alternative interpretation of these correlations is that personality traits have no causal effect on school performance, but that both personality and school performance are determined by the same factor (e.g., aspects of intelligence that are not measured). If this is the case, trying to develop interventions by aim of enhancing certain valued personality traits would not help children to perform better at school.

This possibility relates to literature focusing a mismatch between skills that children develop in low-SES home-environments and demands in school settings (Ellis et al., 2017; Rogoff et al., 2017; Sternberg, 2005; Valencia, 2010). Both personality and cognitive skills required for school performance may have developed in interaction with children's home environments, and therefore show a statistically significant correlation. The question whether the personality actually influences school performance, and whether this influence varies across different levels of students' SES, should be addressed in future research. In order to test this causality, future research could examine whether there are interventions that are able to increase certain traits, such as Openness, and test these interventions' effects on school performance. 


\section{Limitations}

One limitation of our study is the use of test performance and educational attainment as outcome measures. Although educational attainment can be classified as crucial because it is highly predictive for future wages and occupational prestige (e.g., Mayhew, Rockenbach, Bowman, Seifert, \& Wolniak, 2016), other important outcomes, such as psychosocial aspects, also deserve attention. More specifically, we have not examined the relation between Openness and outcomes such as low-SES student's social integration, well-being at school and well-being at home. More research is needed to shed light on this broader picture.

Another limitation of our study is related to our measurement of personality traits. Although research on the five-factor model of personality has shown that the facets underlying the five personality traits are highly correlated, it may be the case that certain facets predict school performance stronger than other facets (O’Connor \& Paunonen, 2007). For example, although in general, Openness is positively related to school performance, it is shown that the facet 'tendency to fantasize', one of the six facets of Openness, is negatively correlated with school performance (De Fruyt, \& Mervielde, 1996). The data analyzed in the current study do not allow us to examine the specific facets underlying personality traits separately. Therefore, the question whether certain facets of Openness are more important in the moderating role of SES in the relation between Openness and school performance, should be addressed in future research.

\section{Conclusion}

We show, with a large and representative longitudinal dataset, that personality traits in late childhood predict educational attainment in middle adolescence, in addition to students' SES and intelligence. Results showed evidence for the independent effect hypothesis with regard to students' test performance: Emotional stability, Openness, and SES appeared to have independent positive relations with test performance. In contrast, we also find evidence for the resource substitution hypotheses with regard to students' educational attainment. Openness appeared to be a stronger predictor of educational attainment at lower levels of students' SES. Therefore, we conclude that for low-SES children, especially the personality trait Openness may compensate for background disadvantage. The differential findings for test performance and educational attainment may suggest that the compensating role of Openness works through teachers' evaluations. Displaying Openness may positively influence the lower teacher expectations that low-SES students are likely to meet. Further research is needed to examine whether it is possible to strengthen students' Openness with educational interventions, and whether these interventions would actually help to diminish the gap in educational attainment between students from different backgrounds. 


\subsection{References}

Akos, P., Lambie, G. W., Milsom, A., \& Gilbert, K. (2007). Early adolescents' aspirations and academic tracking: An exploratory investigation. Professional School Counseling, 11(1), 57-64.

Borghans, L., Duckworth, A. L., Heckman, J. J., \& Ter Weel, B. (2008). The economics and psychology of personality traits. Journal of Human Resources, 43(4), 972-1059.

Bourdieu, P., \& Passeron, J.-C. (1977). Reproduction in education, society and culture. Thousand Oaks, CA: Sage.

Damian, R. I., Su, R., Shanahan, M., Trautwein, U., \& Roberts, B. W. (2015). Can personality traits and intelligence compensate for background disadvantage? Predicting status attainment in adulthood. Journal of Personality and Social Psychology, 109(3), 473.

Davis-Kean, P. E. (2005). The influence of parent education and family income on child achievement: the indirect role of parental expectations and the home environment. Journal of Family Psychology, 19(2), 294.

De Fruyt, F., \& Mervielde, I. (1996). Personality and interests as predictors of educational streaming and achievement. European journal of personality, 10(5), 405-425.

Duckworth, A. L., Weir, D. R., Tsukayama, E., \& Kwok, D. (2012). Who does well in life? Conscientious adults excel in both objective and subjective success. Frontiers in Psychology, 3, 356.

Duncan, G. J., \& Brooks-Gunn, J. (1997). Consequences of growing up poor. New York, NY: Russell Sage Foundation Press.

Dutch inspectorate of education (2018). The State of Education in the Netherlands 2016/2017. Utrecht: Dutch inspectorate of education.

Eamon, M. K. (2002). Effects of poverty on mathematics and reading achievement of young adolescents. The Journal of Early Adolescence, 22(1), 49-74.

Ellis, B. J., Bianchi, J., Griskevicius, V., \& Frankenhuis, W. E. (2017). Beyond risk and protective factors: An adaptation-based approach to resilience. Perspectives on Psychological Science, 12(4), 561-587.

Enders, C. K., \& Bandalos, D. L. (2001). The relative performance of full information maximum likelihood estimation for missing data in structural equation models. Structural Equation Modeling, 8, 430-457.

Entwistle, N. J. (1972). Personality and academic attainment. British Journal of Educational Psychology, 42(2), 137-151.

Evans, G. W., \& Schamberg, M. A. (2009). Childhood poverty, chronic stress, and adult working memory. Proceedings of the National Academy of Sciences, 106(16), 6545-6549.

Gerhardt, M. W., Rode, J. C., \& Peterson, S. J. (2007). Exploring mechanisms in the personalityperformance relationship: Mediating roles of self-management and situational constraints. Personality and Individual Differences, 43(6), 1344-1355.

De Graaf, N. D., De Graaf, P. M., \& Kraaykamp, G. (2000). Parental cultural capital and educational attainment in the Netherlands: A refinement of the cultural capital perspective. Sociology of Education, 73(2), 92-111. 
Hazrati-Viari, A., Rad, A. T., \& Torabi, S. S. (2012). The effect of personality traits on academic performance: The mediating role of academic motivation. Procedia-Social and Behavioral Sciences, 32, 367-371.

Hackman, D. A., \& Farah, M. J. (2009). Socioeconomic status and the developing brain. Trends in Cognitive Sciences, 13(2), 65-73.

Heckman, J. J. (2006). Skill formation and the economics of investing in disadvantaged children. Science, 312(5782), 1900-1902.

Heckman, J. J., \& Kautz, T. (2012). Hard evidence on soft skills. Labour Economics, 19, 451- 464. Jussim, L., Eccles, J., \& Madon, S. (1996). Social perception, social stereotypes, and teacher expectations: Accuracy and the quest for the powerful self-fulfilling prophecy. Advances in Experimental Social Psychology, 28, 281-388.

Johnson, J. A. (2014). Measuring thirty facets of the Five Factor Model with a 120-item public domain inventory: Development of the IPIP-NEO-120. Journal of Research in Personality, 51, 78-89.

Kean, J., \& Reilly, J. (2014). Item response theory. Handbook for Clinical Research: Design, Statistics and Implementation. New York, NY: Demos Medical Publishing.

Lee, V. E., \& Burkam, D. T. (2002). Inequality at the starting gate: Social background differences in achievement as children begin school. Economic Policy Institute, Washington DC.

Mani, A., Mullainathan, S., Shafir, E., \& Zhao, J. (2013). Poverty impedes cognitive function. Science, 341(6149), 976-980.

Mayhew, M. J., Rockenbach, A. B., Bowman, N. A., Seifert, T. A., \& Wolniak, G. C. (2016). How college affects students: 21st Century evidence that higher education works. San Francisco: Jossey-Bass.

McCrae, R. R., \& Costa, P. T., Jr. (1997). Personality trait structure as a human universal. American Psychologist, 52(5), 509.

Merton, R. K. (1968). The Matthew effect in science: The reward and communication systems of science are considered. Science, 159(3810), 56-63.

Mirowsky, J., \& Ross, C. (2003). Education, social status, and health. New York, NY: Aldine De Gruyter.

Moffitt, T. E., Arseneault, L., Belsky, D., Dickson, N., Hancox, R. J., Harrington, H., ... Caspi, A. (2011). A gradient of childhood selfcontrol predicts health, wealth, and public safety. PNAS Proceedings of the National Academy of Sciences of the United States of America, 108, 2693-2698.

Mullainathan, S., \& Sharif, E. (2013). Scarcity: Why having too little means so much. London: Allen Lane.

Noftle, E. E., \& Robins, R. W. (2007). Personality predictors of academic outcomes: big five correlates of GPA and SAT scores. Journal of Personality and Social Psychology, 93(1), 116.

O'Connor, B. P. (2002). A quantitative review of the comprehensiveness of the five-factor model in relation to popular personality inventories. Assessment, 9(2), 188-203.

O'Connor, M. C., \& Paunonen, S. V. (2007). Big Five personality predictors of post-secondary academic performance. Personality and Individual differences, 43(5), 971-990.

Poropat, A. E. (2009). A meta-analysis of the five-factor model of personality and academic performance. Psychological Bulletin, 135, 322-338. 
Raven, J. C., \& Court, J. H. (1998). Raven's progressive matrices and vocabulary scales. Oxford pyschologists Press.

Roberts, B. W., Kuncel, N. R., Shiner, R., Caspi, A., \& Goldberg, L. R. (2007). The power of personality: The comparative validity of personality traits, socioeconomic status, and cognitive ability for predicting important life outcomes. Perspectives on Psychological science, 2(4), 313-345.

Rogoff, B., Coppens, A. D., Alcalá, L., Aceves-Azuara, I., Ruvalcaba, O., López, A., \& Dayton, A. (2017). Noticing learners' strengths through cultural research. Perspectives on Psychological Science, 12, 876-888.

Sijtsma, K. (2009). On the use, the misuse, and the very limited usefulness of Cronbach's alpha. Psychometrika, 74(1), 107.

Shanahan, M. J., Bauldry, S., Roberts, B. W., Macmillan, R., \& Russo, R. (2014). Personality and the reproduction of social class. Social Forces, 93(1), 209-240.

Shiner, R. L., \& Masten, A. S. (2012). Childhood personality as a harbinger of competence and resilience in adulthood. Development and psychopathology, 24(2), 507-528.

Sirin, S. R. (2005). Socioeconomic status and academic achievement: A meta-analytic review of research. Review of educational research, 75(3), 417-453.

Speybroeck, S., Kuppens, S., Van Damme, J., Van Petegem, P., Lamote, C., Boonen, T., \& de Bilde, J. (2012). The role of teachers' expectations in the association between children's SES and performance in kindergarten: A moderated mediation analysis. PloS one, 7(4), e34502.

Sternberg, R. J. (2005). The theory of successful intelligence. Interamerican Journal of Psychology, 39(2), 189-202.

Strenze, T. (2007). Intelligence and socioeconomic success: A meta-analytic review of longitudinal research. Intelligence, 35, 401-426.

Tabachnick, B. G., \& Fidell, L. S. (2001). Using Multivariate Statistics (4th Ed.). Needham Heights, MA: Allyn \& Bacon.

Trizano-Hermosilla, I., \& Alvarado, J. M. (2016). Best alternatives to Cronbach's alpha reliability in realistic conditions: congeneric and asymmetrical measurements. Frontiers in psychology, 7, 769 .

UNESCO Institute for Statistics. (2012). International standard classification of education: ISCED 2011. Montreal: UNESCO Institute for Statistics.

Valencia, R. R. (2010). Dismantling contemporary deficit thinking: Educational thought and practice. New York, NY: Routledge.

Woessmann, L. (2009). International evidence on school tracking: A review. CESifo DICE Report, 7(1), 26-34.

Walberg, H. J., \& Tsai, S. L. (1983). Complementary effects in education. A merican Educational Research Journal, 20(3), 359-373.

White, K. R. (1982). The relation between socioeconomic status and academic achievement. Psychological bulletin, 91(3), 461-481. 



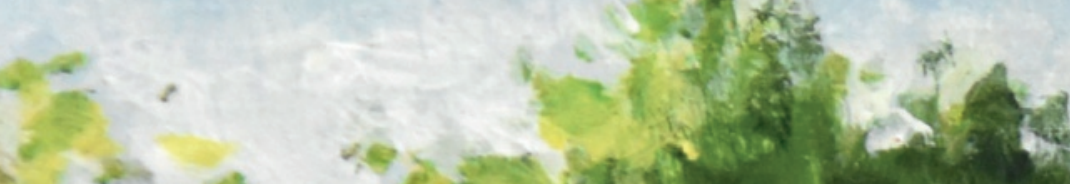

tes is un wat

$2 x-4 x^{2}$

\section{$2=$ \\ SW \\ $x_{2} \cos ^{2}$ \\ $8 x$ \\ $\approx$}

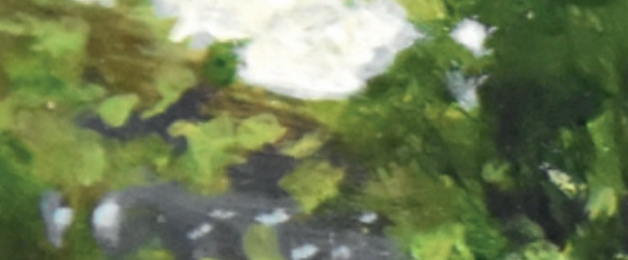

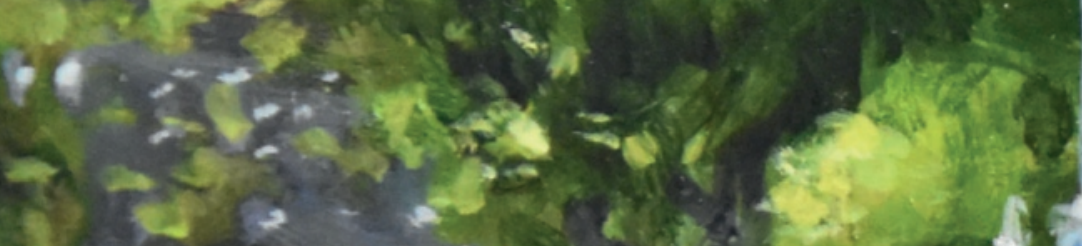

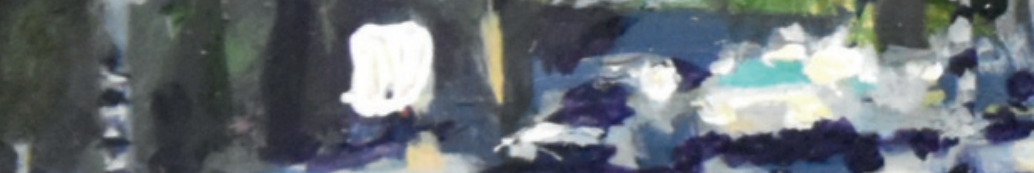

$1 \frac{2}{3}=2$

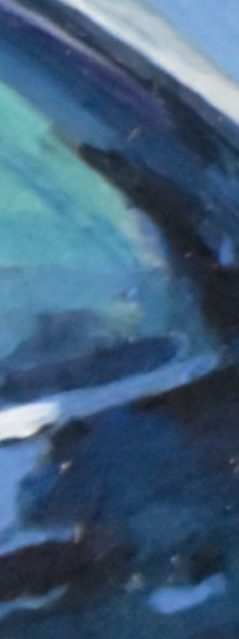




\begin{abstract}
Students from low socioeconomic (SES) backgrounds have a lower chance to attain a degree in the most selective institutions of higher education than high-SES students. These social differences are partly due to low-SES students experiencing psychological barriers during their integration into college, including a lower sense of belonging. Moreover, low-SES students are also more likely to attend colleges that are less selective than their academic credentials would permit (i.e., undermatching), which has negative implications for their salary and job prospects. Little is known, however, about the consequences of undermatching for low-SES students' subjective experience in college. With preregistered analyses of a 6-year longitudinal cohort study of 7,305 students who are eligible for the most selective institutions (aged 16-24), we replicate the finding of psychological barriers that low-SES students may experience in highly selective institutions during their first year in college. Specifically, we show that undermatched low-SES students in less selective colleges experience more satisfaction with their social environment than low-SES students in highly selective institutions throughout their first year. Among high-SES students, in contrast, undermatching predicts lower satisfaction with social aspects in college toward the end of the first year. With regard to academic aspects of college, undermatching does not promote satisfaction, neither for low-SES nor for high-SES students. We conclude that in current higher education settings, socially upwardly mobile students (but not high-SES students) may benefit from undermatching with regard to their social well-being in college, promoting their integration into college. These findings have implications for policy and interventions aimed at preventing dropout from college and promoting upward social mobility.
\end{abstract}




\subsection{Introduction}

Despite decades of policy efforts to promote upward social mobility among youth from low socioeconomic status (low-SES) families, low-SES students remain substantially less likely to attain degrees in the most selective institutions in higher education compared to highSES students (Breen \& Jonsson, 2005; Lucas, 2001; Parker, Schoon, Tsai, Nagy, Trautwein, \& Eccles, 2012; Shavit \& Blossfeld, 1993). In the U.S., 99\% of high-SES students entering university will attain a Bachelor's degree by age 24 , compared to only $21 \%$ of low-SES students (Cahalan \& Perna, 2015). These social differences are partly due to psychological barriers that low-SES students experience when they attend the most selective institutions, enhancing their chance of college retention (Jury et al., 2017; Longden, 2004). In addition, low-SES students that are eligible for the most selective institutions are more likely not to attend a highly selective institution at all, because of undermatching (i.e., attending a less selective institutions than credentials would permit) (Smith, Pender, \& Howell, 2013).

Undermatching is undesirable because of the long-term consequences for students' salary and job prospects (Ovink, Kalogrides, Nanney, \& Delaney, 2017), which reinforces social inequality. Nonetheless, there has been little attention for the subjective experiences of students that undermatch. Whereas experiences of social barriers when low-SES students attend the most selective institutions are well-documented, less is known about subjective experiences when students attend less selective institutions. These experiences are of particular importance in the first year of higher education, when students need to integrate into their new colleges, and when the risk of retention is substantial (Aljohani, 2016).

The current research investigates the development of subjective experiences among first year college students who succeed not to drop out, in terms of their satisfaction with the academic and social environment. Specifically, in six cohorts of students eligible for the most selective institutions ( $N=7,305)$, we examine the satisfaction with the academic and social environment of low-SES students in the most selective institutions compared to high-SES students, and to low-SES students in less selective institutions (undermatched students), both in the beginning and at the end of their first year.

\section{Experiences of psychological barriers}

Jury et al. (2017) have reviewed research about the psychological barriers faced by low-SES students in higher education published within the last 20 years. They conclude that higher education is far from being a culturally neutral environment for low-SES students, because of the prevailing middle- and upper-class norms and cultural codes (see also Stephens, Fryberg, Markus, Johnson, \& Covarrubias, 2012). Low-SES students have to cope with these unknown rules when they attend colleges in higher education, leading to the experience of diverse barriers. More specifically, especially in the first year of higher education, low-SES students are more likely to feel out of place and to experience less sense of belonging than high-SES students (Hinz, 2016; Lee \& Kramer, 2013; Reay, Crozier, \& Clayton, 2010; Ostrove \& Long, 2007). These experiences are related to their salient minority status in higher 
education noticed by others and themselves (Martin, 2015), and with negative stereotypes regarding their competences that low-SES students have to deal with (Durante, Tablante, \& Fiske, 2017). Moreover, they also have lower perceptions of their own competences, reporting lower estimations of their own intelligence and lower self-efficacy than high-SES students (Ivcevic \& Kaufman, 2013; Kudrna, Furnham, \& Swami, 2010). In addition, lowSES students are more likely to feel and express greater emotional distress than high-SES students (Jury et al., 2017). Low-SES students report lower well-being (Padgett, Johnson, \& Pascarella, 2012; Pascarella, Wolniak, Pierson, \& Terenzini, 2003), and higher levels of depression (Steptoe, Tsuda, Tanaka, \& Wardle, 2007) than high-SES students. Research also shows that low-SES students have more difficulties embracing their new identity as college students than high-SES students (Hinz, 2016; Lee \& Kramer, 2013; Reay et al., 2010).

In sum, literature shows that low-SES students experience psychological barriers that contributes to their higher likelihood to drop out, partly driven by unwritten cultural rules and codes in the most selective institutions.

\section{Cultural codes}

Differences in subjective experiences between low- and high-SES students may partly result from interactions between individuals and the university setting, set up in a manner that advantage high-SES students (Browman \& Destin, 2016; Jury et al., 2017; Stephens, Markus, \& Fryberg, 2012). University settings, especially the most selective ones, may promote behavior, attitudes, and speech that are more compatible with norms in high-SES environments than in low-SES environments (Jæger, 2009). According to theories of cultural capital, high-SES teachers and students notice SES-related differences between students from low- and high-SES parents. These cultural differences could lead to lower levels of social integration and even social exclusion of low-SES students in higher education (De Graaf, De Graaf, \& Kraaykamp, 2000; Jæger, 2009; Walpole, 2003). In addition, high-SES students might be more aware of cultural codes familiar to and preferred by lecturers and fellow students in universities, providing high-SES students with social advantages (Lamont \& Lareau, 1988).

A specific cultural difference between low- and high-SES students that seems to play an important role in college, is students' cultural orientation. Whereas high-SES students tend to have independent cultural values (e.g., working independently, prioritizing individual goals above others' goals) promoted in college settings, low-SES students are more likely to embrace interdependent values (e.g., adjusting to others' expectations, focusing on interests for the group) (Stephens, Townsend, \& Dittmann, 2019). The cultural mismatch experiences by low-SES students that results from these cultural values promoted at universities, adds to the psychological barriers in college (Stephens, Fryberg et al., 2012; Stephens et al., 2019). Taken together, cultural norms in college may advantage high-SES students, and may disadvantage low-SES students, particularly in the most selective institutions. 


\section{Undermatching and the experience of barriers}

Low-SES students are more likely to attend a college that is less selective than their credentials would permit (Smith et al., 2013). Whereas in the most selective institutions, low-SES students form a small minority, low-SES students form more substantial part of the population in the less selective institutions of higher education (e.g., Walpole, 2003). For example, in the Netherlands, many more students have a low-SES background in less selective institutions (40\%) than in the most selective institutions (25\%) (Dutch Inspectorate of Education, 2018). These differences in student composition between most selective and less selective institutions may bring along differences in prevailing cultural norms. Studies on minorities in work settings have shown that small groups can overturn established norms if they reach a critical mass (e.g., Kanter, 1977). A recent experimental study indeed showed the existence of a tipping point in the dynamics of changing social conventions. This study estimated that in order to truly change established social conventions minorities have to have a share of more than $25 \%$ (Centola, Becker, Brackbill, \& Baronchelli, 2018). Therefore, the social norms in the less selective institutions may fit better with low-SES students than the norms in the most selective institutions. However, students who attend colleges that are misaligned with their cognitive capacities may experience mismatch with academic aspects, such as the content and structure of the study program (Hoxby \& Turner, 2013; Light \& Strayer, 2000). This academic mismatch may diminish student satisfaction for students, regardless of their socioeconomic backgrounds. In sum, whereas low-SES students may experience fewer social barriers after undermatching, they might experience an academic mismatch in less selective institutions during their integration into college.

\section{First year in college}

Models on student retention predict that the college environment includes an academic and a social system, and students have to integrate in both systems to stay and succeed in college (Aljohani, 2016). The academic environment is the educational context in higher education and includes teaching methods, subject areas, and qualities of lecturers (Aljohani, 2016). The social environment is the social context in higher education, and includes social relationships amongst students, between students and academic staff, and social codes (Jury et al., 2017). Models of student retention also predict that in the beginning of their time in college, students go through a phase of transition in which they adapt to the norms and values of their college (Aljohani, 2016). Student retention in the first year of college can be partly explained by the failure to integrate into the social environment, the academic environment, or both (Aljohani, 2016). A smooth first year in college, in terms of positive subjective experiences with university life, predicts future educational achievement and persistence (Lowe \& Cook, 2003).

\section{Undermatching in the Netherlands}

Research on undermatching in higher education has been mainly conducted in the United States. In the U.S. context, both institutions' selectivity and students' eligibility have to 
be estimated. The reliability of these estimates, and their comparability across studies, are subject to debate (Rodriguez, 2015). In the Netherlands, in contrast, undermatching is easier to determine. First, students can enroll in higher education at two types of institutions: the most selective institutions (science universities) and less selective institutions (universities of applied science). Second, eligibility to these institutions is determined by students' highest level attained during high-school, which is clearly determined. In this study, we take advantage of this clarity by applying data from the educational context in the Netherlands.

\section{The current study}

Subjective experiences of socially mobile students in higher education determine largely their chance to succeed and are therefore important to identify. Whereas low-SES students are more likely to undermatch than high-SES students, little is known about the consequences of undermatching for low-SES students' subjective experience in college. Undermatching may reduce experiences of social mismatch, but at the same time, may induce experiences of academic mismatch. Therefore, in the present study, we compare low-SES students' satisfaction in the most selective institutions with satisfaction of undermatched low-SES students during the first year in higher education. In addition, we investigate the development of low-SES students' satisfaction with their social and academic environment during the first year in higher education in the most selective institutions, and compare their satisfaction with undermatched low-SES students, and high-SES students.

\subsection{Method}

\section{Data}

We preregistered our hypotheses, analyses, sample size, and constructs at the Open Science Framework (https://osf.io/bemj6). A preregistration is a study plan that clearly states, among other things, which statistical analyses are confirmatory and which ones exploratory (Wagenmakers, Wetzels, Borsboom, van der Maas, \& Kievit, 2012). The emerging view in psychological science is that preregistered research is more likely to produce findings that replicate (Chambers, 2013; Nosek \& Lakens, 2014; Nelson, Simmons, \& Simonsohn, 2017).

We used the Start-dataset from the Startmonitor, a large-scale survey in the Netherlands of students' experiences in their first year in higher education. Each year, from 2010 to 2015, researchers randomly selected participants from all higher education institutions in the Netherlands. The resulting dataset contains data from six waves (2010 to 2015) and a total sample of $N=21,160$. From these data, we selected students who (a) were qualified to enter the most selective institutions of tertiary education (according to Dutch law when they have obtained a VWO-diploma); (b) obtained their secondary education diploma in the 
academic year before entering higher education; (c) had not switched between different fields of study and did not drop out, during the first year in higher education. This selection process resulted in a sample of 7,305 participants whose age ranged from 16 to 24 years of age $(M=18.25, S D=0.70)$. The characteristics of this sample are comparable with the demographic composition of first-year Dutch students, with an average age between 18 and 19 years old, and a small overrepresentation of female students (Dutch Inspectorate of Education, 2018).

Undermatching. We compared students who enter the most selective institutions (science universities) with students who enter less selective institutions of higher education (universities of applied science). We selected students eligible for the most selective institutions. This selection minimizes the effects of pre-existent differences in cognitive and social skills developed during secondary education on their experiences in higher education. The Dutch educational system is tracked in high school (secondary education). Diplomas from secondary tracks (VWO is the highest educational track, HAVO is a lower track) each give specific eligibility for either the most selective institutions (science universities) or less selective institutions (universities of applied sciences) in higher education. The most selective institutions are only accessible for students with a VWO diploma. Less selective institutions are open to students with a HAVO or VWO diploma. Our participants were either matched (i.e., enrolled in the most selective institutions) or undermatched (i.e., enrolled in less selective institutions).

SES. We measured socioeconomic status (SES) in two different ways. First, we used the conventional notion of SES as a combination of parental education, income and occupation (SES definition 1). Second, given our focus on students' experience in education, we used a measure of SES that is based solely on parental educational level (SES definition 2). Using both of these measures, rather than a single one, is important because the various components of SES, like parental educational level or income, could affect students' social and academic outcomes through different processes (American Psychological Association, Task Force on Socioeconomic Status, 2007).

SES definition 1 (three categories: low-, middle-, and high-SES) is based on standard income scores (lower than average $=-1$, average $=0$, higher than average $=1$ ), education (both parents have not obtained a diploma in higher education $=-1$, one or both parents obtained diplomas in higher education $=1$ ), and occupation (both parents are unemployed $=-1$, one parent is employed $=0$, both parents are employed $=1$ ). We combined these measures with equal weightings (range -3 to 3 ) and recode them to low-SES ( -3 and -2 ), middle-SES $(-1,0,1)$ and high-SES (2 and 3$)$. In sum, SES definition 1 represents parental educational level, occupation, and income in a composite score. SES definition 2 has two levels: 1) high-SES, one or both parents obtained a diploma in higher education, and 2) low- and middle-SES, both parents have not obtained a diploma in higher education. Hence, SES definition 2 represents parental educational level. 
Satisfaction. In the Startmonitor, participants fill in a questionnaire about their experiences in their first year of higher education. Participants completed this questionnaire twice, once at the beginning of the first academic year (Time 1, September), and once at the end of it (Time 2, June). Five items of this questionnaire reflect students' satisfaction. Of these, three reflect their experiences with the academic environment and two with the social environment. The response scale ranged from 1, very poor, to 10 , excellent. Students were given the prompt "please rate the following aspects of your study program at your institution" in order to assess their perception of their academic environment: (1) the content of the program of study, (2) teaching methods and (3) quality of lecturers. In addition, students rated aspects of their social environment: (1) personal contact with fellow students and (2) personal contact with lecturers. Because we are interested in the distinct results of these items, we decided to analyze them separately.

\subsection{Results}

We provide descriptive statistics in Tables 1 and 2.

TABLE 1 Descriptive Statistics of Dependent Variables (1-5) and Independent Variables (6-10) of Analysis 1 and 2

\begin{tabular}{lcccc}
\hline & $\begin{array}{c}\text { Mean or } \\
\text { percentage }\end{array}$ & SD & Minimum & Maximum \\
\hline 1. Satisfaction with content of study program & 8.05 & 1.02 & 1 & 10 \\
2. Satisfaction with teaching methods & 7.34 & 1.13 & 1 & 10 \\
3. Satisfaction with quality of lecturers & 7.38 & 1.13 & 1 & 10 \\
4. Satisfaction with personal contact with lecturers & 6.79 & 1.70 & 1 & 10 \\
5. Satisfaction with personal contact with fellow students & 7.98 & 1.28 & 1 & 10 \\
6. Average grade final exams secondary education & 7.13 & 0.68 & 5 & 9.5 \\
7. Institution (most selective institutions) & $82 \%$ & & 0 & 1 \\
8. SES (low-SES) (def.1) & $11 \%$ & & 0 & 1 \\
9. SES (low-SES) (def. 2) & $30 \%$ & & 0 & 1 \\
10. Gender (female) & $68 \%$ & & 0 & 1 \\
\hline
\end{tabular}

Note. $N=7,305$

TABLE 2 Correlations amongst SES and Institution

\begin{tabular}{lccc}
\hline & $\begin{array}{c}\text { Institution (most selective } \\
\text { institutions) }\end{array}$ & $\begin{array}{c}\text { SES (low-SES) } \\
\text { (def.1) }\end{array}$ & $\begin{array}{c}\text { SES (low-SES) } \\
\text { (def.2) }\end{array}$ \\
\hline Institution (most selective institutions) & - & - \\
SES (low-SES) (def.1) & $.09^{\star \star}$ & $-.79^{\star *}$ & - \\
SES (low-SES) (def. 2) & $-.10^{\star *}$ & - \\
\hline
\end{tabular}

Note. Kendall's tau, ${ }^{\star} p<.05,{ }^{\star \star} p<.01,{ }^{\star \star \star} p<.001$. 
First, to examine the question whether undermatched takes along benefits for low-SES students in terms of satisfaction, we examined whether there are differences between undermatched low-SES students and matched low-SES students in satisfaction at the end of the first year in college. We selected low-SES students (def. 1), and performed five separate linear regression analyses with satisfaction at Time 2 (five items) as the response variable, institution ( $1=$ most selective institutions, $0=$ less selective institutions) as the predictor variable and average grade on final exams in secondary education, age and gender ( $1=$ female, $0=$ male) as covariates. We present the results of five models predicting satisfaction in Table 3. Because our units of measurement have a meaningful interpretation, we report unstandardized regression coefficients.

Regression analyses showed no significant difference between low-SES students' satisfaction in the most selective institutions compared to low-SES students' satisfaction in less selective institutions with the content of the study program, teaching methods, and quality of lecturers. However, low-SES students' satisfaction was significantly higher after undermatching regarding personal contact with lecturers, and personal contact with fellow students. Including covariates led to similar statistically significant results, and results showed the same pattern when applying SES definition 2 (results not shown). These results indicate that undermatching may take along benefits for low-SES students regarding their satisfaction, but only with regard to social aspects in college.

\section{SES, undermatch, and satisfaction}

Second, we examined whether the relation between undermatching and satisfaction with several aspects in college is moderated by students' SES. Using the whole sample including students from all SES-backgrounds $(\mathrm{N}=7,305)$, we performed five separate linear regression analyses, with satisfaction at Time 2 (five items) as the response variable, institution ( 1 = most selective institutions, 0 = less selective institutions), SES-background, and institution $\times$ SES-background interaction as predictor variables, and average grade on final exams in secondary education, age, and gender as covariates. We present the results of these five models predicting satisfaction in Table 4 .

Results showed no significant interaction between undermatching and SES on students' satisfaction at the end of their first academic year on the content of the study program, teaching methods, quality of lecturers, and personal contact with lecturers. These results do not indicate a moderating role of SES in the relation between undermatching and satisfaction with these aspects in college. 
TABLE 3 Results from Linear Regression Models Predicting Satisfaction among Low-SES Students in Higher Education (SES definition 1)

\begin{tabular}{|c|c|c|c|c|}
\hline \multirow[b]{2}{*}{ Predictor } & \multicolumn{2}{|c|}{ Model 1 (without covariates) } & \multicolumn{2}{|c|}{ Model 2 (with covariates) } \\
\hline & $b$ & $95 \% \mathrm{Cl}$ & $b$ & $95 \% \mathrm{Cl}$ \\
\hline & \multicolumn{4}{|c|}{ Outcome: satisfaction with content of the study program } \\
\hline Intercept & $8.03^{\star \star}$ & {$[7.76,8.30]$} & $5.62^{\star \star}$ & {$[2.64,8.60]$} \\
\hline Institution (most selective) & -0.04 & {$[-0.33,0.25]$} & -0.12 & {$[-0.41,0.17]$} \\
\hline Average grade final exams & - & - & $0.26^{\star \star}$ & {$[0.09,0.43]$} \\
\hline Age & - & - & 0.04 & {$[-0.10,0.18]$} \\
\hline \multirow[t]{2}{*}{ Gender (female) } & - & - & -0.12 & {$[-0.34,0.11]$} \\
\hline & \multicolumn{4}{|c|}{ Outcome: satisfaction with teaching methods } \\
\hline Intercept & $7.30^{\star \star}$ & {$[7.02,7.58]$} & $8.90^{\star *}$ & {$[5.76,12.03]$} \\
\hline Institution (most selective) & -0.02 & {$[-0.32,0.28]$} & -0.05 & {$[-0.36,0.25]$} \\
\hline Average grade final exams & - & - & 0.07 & {$[-0.11,0.25]$} \\
\hline Age & - & - & -0.11 & {$[-0.26,0.03]$} \\
\hline \multirow[t]{2}{*}{ Gender (female) } & - & - & -0.03 & {$[-0.27,0.2]$} \\
\hline & \multicolumn{4}{|c|}{ Outcome: satisfaction with quality of lecturers } \\
\hline Intercept & $7.21^{\star \star}$ & {$[6.94,7.48]$} & $6.17^{\star \star}$ & {$[3.11,9.23]$} \\
\hline Institution (most selective) & 0.25 & {$[-0.05,0.54]$} & 0.18 & {$[-0.12,0.48]$} \\
\hline Average grade final exams & - & - & 0.10 & {$[-0.08,0.28]$} \\
\hline Age & - & - & 0.03 & {$[-0.11,0.17]$} \\
\hline \multirow[t]{2}{*}{ Gender (female) } & - & - & $-0.26^{\star}$ & {$[-0.49,-0.02]$} \\
\hline & \multicolumn{4}{|c|}{ Outcome: satisfaction with personal contact with lecturers } \\
\hline Intercept & $7.84^{\star *}$ & {$[7.42,8.26]$} & $6.69^{*}$ & {$[1.92,11.45]$} \\
\hline Institution (most selective) & $-1.27^{\star \star}$ & {$[-1.73,-0.81]$} & $-1.33^{\star \star}$ & {$[-1.80,-0.86]$} \\
\hline Average grade final exams & - & - & 0.15 & {$[-0.13,0.42]$} \\
\hline Age & - & - & 0.02 & {$[-0.21,0.24]$} \\
\hline \multirow[t]{2}{*}{ Gender (female) } & - & - & -0.13 & {$[-0.50,0.23]$} \\
\hline & \multicolumn{4}{|c|}{ Outcome: satisfaction with personal contact with fellow students } \\
\hline Intercept & $8.49^{\star \star}$ & {$[8.16,8.83]$} & $6.95^{\star \star}$ & {$[3.15,10.75]$} \\
\hline Institution (most selective) & $-0.70^{\star \star}$ & {$[-1.07,-0.34]$} & $-0.73^{\star \star}$ & {$[-1.10,-0.36]$} \\
\hline Average grade final exams & - & - & 0.16 & {$[-0.06,0.38]$} \\
\hline Age & - & - & 0.02 & {$[-0.15,0.20]$} \\
\hline Gender (female) & - & - & 0.06 & {$[-0.23,0.35]$} \\
\hline
\end{tabular}

Note. $\mathrm{Cl}=$ confidence interval, ${ }^{*} p<.05,{ }^{\star *} p<.01$. 
TABLE 4 Results from Linear Regression Models Predicting Satisfaction among Students from All SES-Backgrounds in Higher Education (SES definition 1)

\begin{tabular}{|c|c|c|c|c|}
\hline \multirow[b]{2}{*}{ Predictor } & \multicolumn{2}{|c|}{ Model 1 (without covariates) } & \multicolumn{2}{|c|}{ Model 2 (with covariates) } \\
\hline & $b$ & $95 \% \mathrm{Cl}$ & $b$ & $95 \% \mathrm{Cl}$ \\
\hline & \multicolumn{4}{|c|}{ Outcome: satisfaction with content of the study program } \\
\hline Intercept & $7.87^{\star \star}$ & {$[7.76,7.98]$} & $6.62^{\star \star}$ & {$[5.62,7.62]$} \\
\hline Institution (most selective) & $0.24^{\star \star}$ & {$[0.12,0.35]$} & $0.16^{\star}$ & {$[0.05,0.28]$} \\
\hline SES (low-SES) & 0.16 & {$[-0.11,0.43]$} & 0.14 & {$[-0.12,0.41]$} \\
\hline Institution $\times$ SES & -0.27 & {$[-0.56,0.02]$} & -0.23 & {$[-0.52,0.06]$} \\
\hline Average grade final exams & - & - & $0.16^{\star \star}$ & {$[0.11,0.21]$} \\
\hline Age & - & - & 0.01 & {$[-0.04,0.06]$} \\
\hline \multirow[t]{2}{*}{ Gender (female) } & - & - & $0.07^{\star}$ & {$[0.00,0.14]$} \\
\hline & \multicolumn{4}{|c|}{ Outcome: satisfaction with teaching methods } \\
\hline Intercept & $7.19^{\star \star}$ & {$[7.08,7.31]$} & $7.03^{\star \star}$ & {$[5.93,8.12]$} \\
\hline Institution (most selective) & $0.21^{\star \star}$ & {$[0.09,0.34]$} & $0.16^{\star}$ & {$[0.03,0.29]$} \\
\hline SES (low-SES) & 0.10 & {$[-0.19,0.40]$} & 0.10 & {$[-0.19,0.39]$} \\
\hline Institution x SES & -0.23 & {$[-0.55,0.09]$} & -0.20 & {$[-0.52,0.12]$} \\
\hline Average grade final exams & - & - & $0.10^{\star \star}$ & {$[0.04,0.15]$} \\
\hline Age & - & - & -0.03 & {$[-0.08,0.02]$} \\
\hline \multirow[t]{2}{*}{ Gender (female) } & - & - & 0.04 & {$[-0.04,0.11]$} \\
\hline & \multicolumn{4}{|c|}{ Outcome: satisfaction with quality of lecturers } \\
\hline Intercept & $7.21^{\star \star}$ & {$[7.09,7.33]$} & $5.38^{\star \star}$ & {$[4.29,6.47]$} \\
\hline Institution (most selective) & $0.25^{\star \star}$ & {$[0.13,0.37]$} & $0.17^{\star}$ & {$[0.04,0.29]$} \\
\hline SES (low-SES) & 0.00 & {$[-0.29,0.29]$} & -0.01 & {$[-0.30,0.28]$} \\
\hline Institution x SES & 0.00 & {$[-0.32,0.31]$} & 0.04 & {$[-0.28,0.35]$} \\
\hline Average grade final exams & - & - & 0.17 & {$[0.11,0.22]$} \\
\hline Age & - & - & $0.04^{*}$ & {$[-0.01,0.09]$} \\
\hline \multirow[t]{2}{*}{ Gender (female) } & - & - & -0.03 & {$[-0.10,0.05]$} \\
\hline & \multicolumn{4}{|c|}{ Outcome: satisfaction with personal contact with lecturers } \\
\hline Intercept & $7.56^{\star \star}$ & {$[7.38,7.74]$} & $4 \cdot 77^{\star \star}$ & {$[3.12,6.42]$} \\
\hline Institution (most selective) & $-0.92^{\star \star}$ & {$[-1.10,-0.73]$} & $-1.07^{\star \star}$ & {$[-1.26,-0.87]$} \\
\hline SES (low-SES) & 0.28 & {$[-0.17,0.72]$} & 0.27 & {$[-0.17,0.71]$} \\
\hline Institution x SES & -0.35 & {$[-0.83,0.13]$} & -0.29 & {$[-0.77,0.18]$} \\
\hline Average grade final exams & - & - & $0.28^{\star \star}$ & {$[0.20,0.36]$} \\
\hline Age & - & - & 0.05 & {$[-0.03,0.13]$} \\
\hline \multirow[t]{2}{*}{ Gender (female) } & - & - & $-0.16^{\star}$ & {$[-0.25,-0.02]$} \\
\hline & \multicolumn{4}{|c|}{ Outcome: satisfaction with personal contact with fellow students } \\
\hline Intercept & $8.20^{\star \star}$ & {$[8.06,8.33]$} & $7 \cdot 99^{\star \star}$ & {$[6.73,9.25]$} \\
\hline Institution (most selective) & $-0.25^{\star \star}$ & {$[-0.39,-0.10]$} & $-0.31^{\star \star}$ & {$[-0.46,-0.16]$} \\
\hline SES (low-SES) & 0.30 & {$[-0.04,0.63]$} & 0.29 & {$[-0.04,0.63]$} \\
\hline Institution x SES & $-0.46^{\star}$ & {$[-0.82,-0.09]$} & $-0.42^{\star}$ & {$[-0.79,-0.06]$} \\
\hline Average grade final exams & - & - & $0.11^{\star \star}$ & {$[0.05,0.17]$} \\
\hline Age & - & - & -0.03 & {$[-0.09,0.03]$} \\
\hline Gender (female) & - & - & $0.01^{*}$ & {$[-0.07,0.10]$} \\
\hline
\end{tabular}

Note. $\mathrm{Cl}=$ confidence interval, ${ }^{\star} p<.05,{ }^{* \star} p<.01$. 
There was, however, a significant interaction between institution and SES on students' rating on personal contact with fellow students (see also Figure 1). Follow-up analyses of simple effects show that undermatched low-SES (SES definition 1) students were more satisfied with personal contact with fellow students than matched low-SES students in the most selective institutions, $b=0.73, t(432)=3.86, p<.001,95 \% \mathrm{Cl}=[0.36,1.10$, ], and this positive relation between undermatching and satisfaction is stronger than among high-SES students $(b=0.25, t(2454)=2.66, p=.008,95 \% \mathrm{Cl}=[0.07,0.43]$. We found no differences between low- and high SES students within the most selective institutions $(b=-0.13, t(2606)=1.73, p=.083)$. However, when applying SES definition 2, we found differences within the most selective institutions: low-SES youth were less satisfied with personal contact with fellow students than students from high-SES backgrounds, $b=-0.09$, $t(5813)=2.43, p=.015,95 \% \mathrm{Cl}=[-0.17,-0.02]$. These results suggest that undermatching is related to more satisfaction with social aspects in college, especially among low-SES students.

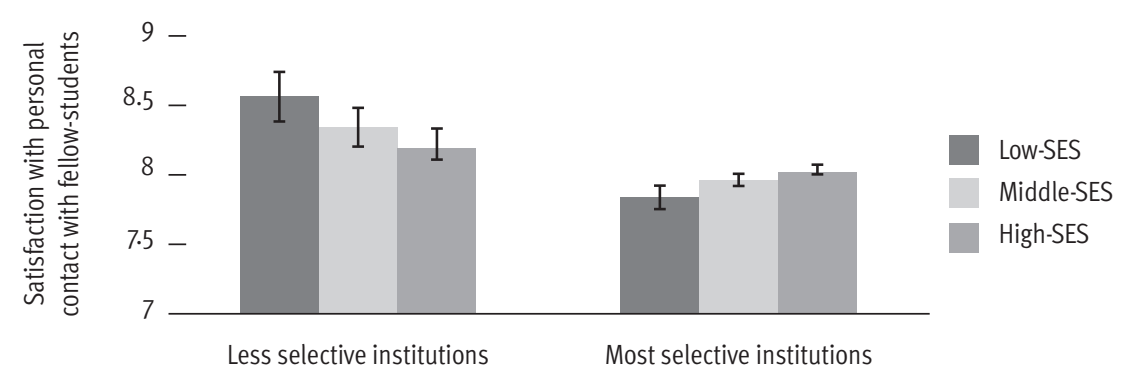

FIGURE 1 LoW-, middle-, and high-SES (definition 1) students' satisfaction with personal contact with fellow students (scale 1-10) in the most selective institutions and less selective institutions in higher education. Error bars represent 1 SEM.

\section{Development of satisfaction in the first academic year}

Third, we examined whether the relation between undermatching and the development of satisfaction with academic and social aspects in the course of the first year of higher education, is moderated by students' SES (SES def 1). We selected respondents ( $N=$ 1,757) who filled in the questionnaire twice (Time 1 and Time 2). We performed five separate repeated measures ANCOVAs with satisfaction at Time 1 and Time 2 (five items) as the response variables, time as the within-subject factor, SES and institution as the independent variable, and average grade on final exams in secondary education, age and gender as covariates. We show descriptive statistics in Table 5. 
TABLE 5 Descriptive Statistics of Dependent Variables (1-5) and Independent Variables (6-10) of Analysis 3

\begin{tabular}{lcccc}
\hline & $\begin{array}{c}\text { Mean or } \\
\text { percentage }\end{array}$ & SD & Minimum & Maximum \\
\hline 1. Satisfaction with content of study program (time 1) & 8.30 & 0.99 & 1 & 10 \\
1. Satisfaction with content of study program (time 2) & 8.03 & 1.06 & 2 & 10 \\
2. Satisfaction with teaching methods (time 1) & 7.48 & 1.07 & 1 & 10 \\
2. Satisfaction with teaching methods (time 2) & 7.31 & 1.12 & 1 & 10 \\
3. Satisfaction with quality of lecturers (time 1) & 7.74 & 1.01 & 3 & 10 \\
3. Satisfaction with quality of lecturers (time 2) & 7.37 & 1.15 & 1 & 10 \\
4. Satisfaction with personal contact with lecturers (time 1) & 6.85 & 1.57 & 1 & 10 \\
4. Satisfaction with personal contact with lecturers (time 2) & 6.69 & 1.69 & 1 & 10 \\
5. Satisfaction with personal contact with fellow students (time 1) & 8.22 & 1.16 & 1 & 10 \\
5. Satisfaction with personal contact with fellow students (time 2) & 7.98 & 1.25 & 1 & 10 \\
6. Average grade final exams secondary education & 7.23 & 0.68 & 5.5 & 9.31 \\
7. Institution (most selective) & $79 \%$ & & 0 & 1 \\
8. SES (low-SES) (def.1) & $11 \%$ & & 0 & 1 \\
9. SES (low-SES) (def. 2) & $31 \%$ & & 0 & 1 \\
10. Gender (female) & $64 \%$ & & 0 & 1 \\
\hline
\end{tabular}

Note. $N=1,757$

Repeated measures ANCOVA showed no significant interaction between educational level, SES and time on students' satisfaction on the content of the study program, $F(1,810)=$ 2.61, $p=.106$, teaching methods, $F(1,810)=0.66, p=.418$, quality of lecturers, $F(1,810)$ $=0.27, p=.990$, personal contact with lecturers, $F(1,810)=0.55, p=.458$, and personal contact with fellow students, $F(1,810)=2.28, p=.132$ when applying SES definition 1. By contrast, when applying SES definition 2, we found a significant interaction between institution, SES and time for satisfaction with personal contact with fellow students, $F(1$, $1431)=7.10, p=.008$, and this relation remained significant after controlling for average grade on final exam in secondary education, age, and gender. Follow-up analyses showed no interaction between institution and time within low-SES students, $F(1,408)=2.19$, $p=.140$, suggesting that during the first year, both in the beginning end the end, lowSES students in the most selective institutions are less satisfied with personal contact with fellow students compared to undermatched low-SES students. In addition, analyses showed no interaction between institution and time within the most selective institutions, $F(1,1282)=0.02, p=.891$, and suggest that high-SES students are more satisfied with personal contact with fellow students than low-SES students, a difference that remains stable during the first year in higher education (see Figure 2). Interestingly, we found a significant interaction between institution and time among high-SES students, $F(1,1020)$ $=6.53, p<.01$, suggesting that high-SES students' satisfaction diminishes more among undermatched high-SES students than among high-SES students in the most selective institutions. 
These results suggest that the relation between undermatching and development of satisfaction during the first year in higher education is not moderated by students' SES for aspects regarding the academic environment. However, with regard to satisfaction with personal contact with fellow students, undermatching appears to protect the development of low-students' satisfaction whereas it diminishes the development of high-SES students' satisfaction.

In addition to our confirmatory analyses, we conducted exploratory analyses testing the moderating roles of gender and subject area of the study program (science and technology versus other subject areas). We found no moderating effects of gender or subject area in the interaction between SES-background and institution on satisfaction with the social environment.

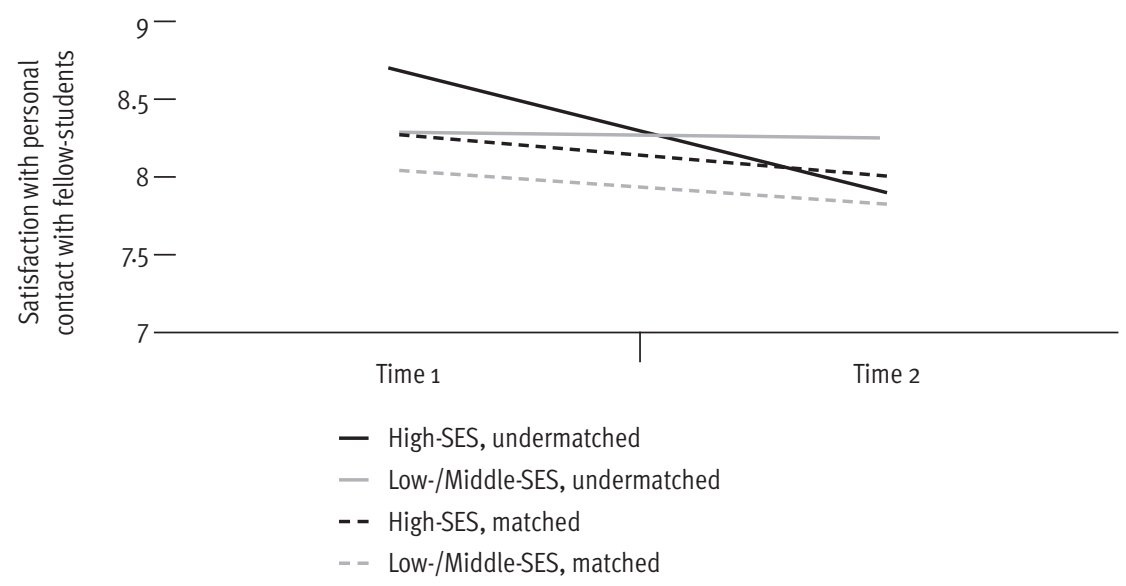

FIGURE 2 LOW-, middle-, and high-SES students' satisfaction with personal contact with fellow students (scale 1-10) in the most selective institutions and less selective institutions (undermatching) of higher education: development during the first academic year.

\subsection{Discussion}

Although societies promote upward social mobility by enabling anyone with ability and motivation to succeed at the highest educational level, low-SES students are still less likely to attain degrees in the most selective institutions. Undermatching, when students attend less selective institutions than their credentials would permit, more often occurs among low-SES students; even with eligibility for the most selective institutions, low-SES students are more likely to make less ambitious educational decisions than high-SES students (Tieben \& Wolbers, 2010). In addition, it is well documented that when low-SES students attend highly selective institutions, they may experience social barriers during their integration in college (Jury et al., 2017), enhancing their risk on retention in their first year in college (Aljohani, 2016; Longden, 2004). 
However, less is known about the relation between undermatching and students' subjective experiences during this important phase in their educational career. From an academic misalignment perspective, regardless of their social background, students who are undermatched may experience an academic mismatch between their capacities and the demands regarding academic aspects (e.g., a less challenging study program) (Light \& Strayer, 2000). Nevertheless, from a social mismatch perspective, low-SES student may benefit from undermatching.

In the current research, we replicated the well-documented finding regarding the social barriers that low-SES students may experience in highly selective institutions. Our study shows that low-SES students in their first year in highly selective institutions, experience less satisfaction with their social environment than their high-SES fellow students. These findings illustrate the well-known cost of upward social mobility for low-SES youth entering the most prestigious level of higher education. In addition, we showed that the differences in satisfaction with social aspects in college between matched and undermatched lowSES students are substantial: among low-SES students, enrollment in the most selective institutions amounts to 1.33 less satisfaction with personal contact with lecturers, and 0.73 less satisfaction with personal contact with fellow students, on a 10-point scale. These findings indicate that from the beginning to the end of the first year, undermatched low-SES students suffer less, or not at all, from social barriers. In contrast, among highSES students, undermatching does show a negative relation with the development of satisfaction with the social environment during the first year.

These patterns and are not found for subjective experiences with the academic environment. The present study shows no differences in satisfaction with the academic environment between matched and undermatched low-SES students, nor a moderating role of students' SES in this relation. Although research on consequences of undermatching predicts an academic mismatch after undermatching, our study has not provided support for a negative relation between undermatching and satisfaction with academic matters in the first year in higher education.

These findings add to literature about undermatching in several ways. First, although undermatching is generally considered as a negative outcome because of the long-term consequences for wages and job opportunities (Ovink et al., 2017), we show that undermatching may have positive consequences on the short term. Undermatching may be a way for low-SES students to circumvent the social barriers in highly selective institutions. The enhanced satisfaction with the social environment in less selective institutions might stimulate low-SES students' chance to attain a college degree, although in a less selective institution. Still, undermatching appeared not to yield these advantages for high-SES students.

Second, investigating factors and processes that determine social selectivity is important because enrollment decisions in the transition to higher education have consequences for students' academic development and future financial prospects (Mayhew, Rockenbach, Bowman, Seifert, \& Wolniak, 2016; Ovink et al., 2017). Low-SES students tend to choose the 
less selective institution, even in educational systems where enrollment in all institutions of higher education are equally expensive (Parker et al., 2012; Tieben \& Wolbers, 2010; Smith et al., 2013). Research on students' decisions during transitions to higher education underscores the importance of adolescents' familiarity with the most selective institutions in guiding them toward these institutions. Low-SES youth have a harder time envisioning their own attendance in the most selective institutions due to concerns about their match with the academic and social environment (Hossler \& Gallagher, 1987; Radford, 2013). Low-SES youth's choice for a less selective institution may in part be driven by the belief that they will experience higher well-being in a less selective institution due to their better fit with the social environment, even though choosing a less selective institution leads to lower educational attainment and lower income in the long term. Our results suggest that low-SES youth's considerations might be valid with regard to their match with social environment in college.

Third, our findings add to a growing literature suggesting that there are costs to upward social mobility. Some studies report a negative impact of upward social mobility on lowSES youth's health (Houle, 2011; House, 1974; Rahe, 1968). In addition, whereas among low-SES youth high levels of self-control lead to more academic success, self-control also seems to undermine their health and accelerate epigenetic aging (Brody et al., 2013; Chen, Miller, Brody, \& Lei, 2014; Miller, Yu, Chen, \& Brody, 2015). Moreover, adverse influence of early stressful experiences may be increased by striving for upward social mobility (Brody et al., 2013; Wickrama, O’Neal, \& Lee, 2016). These adverse outcomes of upward social mobility might be caused by a mismatch with the environment (Brody et al., 2013; Frankenhuis \& Del Giudice, 2012). We show that low-SES youth in the most selective institutions experience social barriers, both in the beginning and end of the first year, but find no evidence for academic barriers. Accordingly, social barriers might be particularly important to understanding the adverse outcomes associated with upward social mobility.

\section{Experiencing social mismatch}

Our results did not indicate social barriers for low-SES students in the less selective institutions of higher education. The composition of the student population could be pivotal. Whereas in the most selective institutions, low SES students form a small minority, low-SES students form more substantial part of the population in the less selective institutions of higher education (Radford, 2013; Walpole, 2003). In the Netherlands, lowSES students form around $40 \%$ of the student population in less selective institutions, in contrast with $25 \%$ in the highly selective institutions (Dutch inspectorate of education, 2018). Studies on minorities have shown that in minorities have to have a share of more than 25 percent to be able to overturn established norms (Centola et al., 2018). Therefore, the larger share of low-SES students in less selective institutions may promote social norms in the less selective institutions that fit better with low-SES students than the norms in the most selective institutions. 
A basic social process is people's tendency to associate and bond with similar others (McPherson, Smith-Lovin, \& Cook, 2001). Students in the same level of higher education are more likely to be similar on a number of characteristics, including skills, IQ, academic interests and ambitions. In addition, students can be similar in their socioeconomic backgrounds. Our results suggest that similarity in socioeconomic backgrounds might be a more important determinant of well-being than having similar academic characteristics as fellow students. This finding suggests a significant role of social background that may explain low-SES youth's less ambitious decisions during transitions, and costs of upward social mobility.

Our results also suggest a social mismatch for high-SES students in the less selective educational institutions of higher education. Although the experiences of high-SES students were not the focus of our investigation, this result evokes challenging questions about the role of social background in educational attainment more broadly. For example, policies typically promote students choosing and accomplishing educational institutions that fit with their talents and ambitions, regardless of their social background. However, social mismatch costs for high-SES students entering less selective institutions might function as a barrier to entering and accomplishing a less selective institution, even if these institutions best fit their capacities.

\section{Interventions supporting low-SES students}

Interventions that facilitate upward social mobility may inadvertently entail costs as well benefits. It is a multifaceted political question whether the benefits (e.g., academic success, better job perspectives) outweigh the costs (e.g., reduced well-being, compromised health and accelerated aging). The goal of this study is to contribute to the understanding of forces that play a role when low-SES youth is upwardly mobile through education. This understanding could help to answer questions about how to facilitate the success of students with diverse needs, expectations, and backgrounds. Hence, research on upward social mobility helps to paint a clearer picture of both costs and benefits, contributing to the development of appropriate interventions that do justice to the gamut of experiences of target populations.

Many policies and interventions have focused mainly on addressing academic or economic barriers to higher education. In our sample, low-SES students did not seem to experience a mismatch with the academic environment in the most selective institutions, possibly because previous policies have successfully removed this barrier. However, social barriers might also determine who enter and complete the most selective institutions and such barriers have been targets of recent experimental interventions, with promising initial results (Okonofua, Paunesku, \& Walton, 2016; Yeager et al., 2016; Stephens, Hamedani, \& Destin, 2014). Some of these interventions are based on the idea that disadvantaged students' and teachers' mindsets (beliefs and expectations) can produce a 'cascade of selffulfilling prophecies', resulting in disadvantaged youth spiraling toward negative outcomes. By changing negative mindsets, these interventions enhanced social and academic 
integration, dramatically reducing suspension and dropout rates of disadvantaged youth. These studies show the significance of addressing social barriers in order to facilitate academic achievement among low-SES students. Interventions that focus on removing the social barriers that low-SES youth experience in the most selective institutions could well be a productive avenue to promoting equal developmental opportunities for individuals from diverse social backgrounds. However, in order to understand both the benefits and the costs of socially upward mobility via the most selective institutions, we need more insight into the long-term consequences of the social barriers that low-SES students experience in these institutions and the consequences of these barriers for their educational achievement, health, and well-being.

\section{Limitations}

Our results suggest that parental education (SES definition 2) is more important in predicting social barriers for low-SES students in the most selective institutions than the concurrence of parental education, income and occupation (SES definition 1). This finding resonates with the cultural capital hypothesis, which proposes that non-financial social assets (such as parental educational level) are key to parents' abilities to support their children in higher education. However, we were not able to examine the possible contribution of parental wealth (e.g. housing, car) as well as the subjective experience of students' social status in this study.

We have focused on SES as a source of mismatch, without being able to tease SES apart from other factors that may be correlated with SES, and which might produce mismatch. Ethnicity, which is associated with personal characteristics (such as skin color) and culture (such as behavioral norms), might be important. Both SES and ethnicity are known to be related to stigmatization of academic abilities, and intersectionality between low-SES and ethnic minority-backgrounds can double this stigmatization (Ivcevic \& Kaufman, 2013). Our data set does not include refined data about ethnicity, hence does not allow us to examine this factor and to tease it apart from SES. The intersectionality between SES and ethnicity is an important avenue for future research.

\section{Conclusion}

Despite policy efforts to make highly selective institutions accessible or students from all socioeconomic background, low-SES students are less likely to attain a degree at this level (Breen \& Jonsson, 2005; Lucas, 2001; Parker et al., 2012; Shavit \& Blossfeld, 1993). This inequality is partly related to experiences of barriers in the most selective institutions, leading to early retention (Jury et al., 2017; Longden, 2004). In addition, this inequality is related to the higher likelihood among low-SES students to attend colleges that are less selective than their credentials would permit (i.e., undermatching) (Smith et al., 2013). Whereas subjective experiences during the integration in college are important predictors of performance and degree completion (Jury et al., 2017), little is known about the relation between undermatching and low-SES students' subjective experience in their 
first year in college. This study shows with preregistered analyses of a 6-year longitudinal cohort study of 7,305 students who are eligible for the most selective institutions (aged 16-24), the well-documented social barriers that low-SES students experience in highly selective colleges. Interestingly, we also show that these barriers seem to be absent in less selective institutions during the whole first year in college, when low-SES students are undermatched. On the contrary, for high-SES students, we found that undermatching predicts decreased satisfaction with social aspects in college toward the end of the first year. In addition, with regard to academic aspects of college, undermatching does not promote positive experiences, neither for low-SES nor for high-SES students. We conclude that, although undermatching predicts less positive integration in the social environment for high-SES students, it may promote integration during the first year in college among low-SES students. Therefore, undermatching may be a way to prevent early retention, but only for low-SES students. 


\subsection{References}

Aljohani, O. (2016). A comprehensive review of the major studies and theoretical models of student retention in higher education. Higher Education Studies, 6(2), 1-18.

American Psychological Association,Task Force on Socioeconomic Status. (2007). Report of the APA Task Force on Socioeconomic Status. Washington, DC.

Breen, R., \& Jonsson, J. O. (2005). Inequality of opportunity in comparative perspective: Recent research on educational attainment and social mobility. Annual Review of Sociology, 31, 223-243.

Brody, G. H., Yu, T., Chen, Y. F., Kogan, S. M., Evans, G. W., Beach, S. R., \& Philibert, R. A. (2013). Cumulative socioeconomic status risk, allostatic load, and adjustment: A prospective latent profile analysis with contextual and genetic protective factors. Developmental Psychology, 49(5), 913-927.

Browman, A. S., \& Destin, M. (2016). The effects of a warm or chilly climate toward socioeconomic diversity on academic motivation and self-concept. Personality and Social Psychology Bulletin, 42(2), 172-187. doi:10.1177/0146167215619379

Cahalan, M., \& Perna, L. (2015). Indicators of Higher Education Equity in the United States: 45 Year Trend Report. Pell Institute for the Study of Opportunity in Higher Education.

Centola, D., Becker, J., Brackbill, D., \& Baronchelli, A. (2018). Experimental evidence for tipping points in social convention. Science, 360(6393), 1116-1119.

Chambers, C. D. (2013). Registered reports: A new publishing initiative at Cortex. Cortex, 49, 609-610.

Chen, E., Miller, G. E., Brody, G. H., \& Lei, M. (2014). Neighborhood poverty, college attendance, and diverging profiles of substance use and allostatic load in rural African American youth. Clinical Psychological Science, 3(5), 675-685.

De Graaf, N. D., De Graaf, P. M., \& Kraaykamp, G. (2000). Parental cultural capital and educational attainment in the Netherlands: A refinement of the cultural capital perspective. Sociology of Education, 73(2), 92-111.

Dutch inspectorate of education (2018). The State of Education in the Netherlands 2016/2017. Utrecht: Dutch inspectorate of education.

Durante, F., Tablante, C., \& Fiske, S. T. (2017). Poor but warm, rich but cold (and competent): Social classes in the stereotype content model. Journal of Social issue, 73(1), 138-157.

Frankenhuis, W. E., \& Del Giudice, M. (2012). When do adaptive developmental mechanisms yield maladaptive outcomes? Developmental Psychology, 48(3), 628-642.

Hinz, S. E. (2016). Upwardly mobile: Attitudes toward the class transition among firstgeneration college students. Journal of College Student Development, 22(3), 285-299.

Hossler, D., \& Gallagher, K. S. (1987). Studying student college choice: A three-phase model and the implications for policymakers. College and university, 62(3), 207-221.

Houle, J. N. (2011). The psychological impact of intragenerational social class mobility. Social Science Research, 40(3), 757-772.

House, J. S. (1974). Occupational stress and coronary heart disease: A review and theoretical integration. Journal of Health and Social Behavior, 15(1), 12-27. 
Hoxby, C. M., \& Turner, S. (2013). Expanding college opportunities for high achieving, low income students (SIEPR 12-014). Stanford, CA: Stanford Institute for Economic Policy Research.

Ivcevic, Z., \& Kaufman, J. C. (2013). The can and cannot do attitude: How self-estimates of ability vary across ethnic and socioeconomic groups. Learning and Individual Differences, 27, 144-148.

Jæger, M. M. (2009). Equal access but unequal outcomes: Cultural capital and educational choice in a meritocratic society. Social Forces, 87(4), 1943-1971.

Jury, M., Smeding, A., Stephens, N. M., Nelson, J. E., Aelenei, C., \& Darnon, C. (2017). The experience of low SES students in higher education: Psychological barriers to success and interventions to reduce social class inequality. Journal of Social Issues, 73(1), 23-41.

Kanter, R. M. (1977). (1977b). Some effects of proportions on group life: Skewed sex ratios and responses to token women. American Journal of Sociology, 82, 965-990.

Kudrna, L., Furnham, A., \& Swami, V. (2010). The influence of social class salience on selfassessed intelligence. Social Behavior and Personality: An International Journal, 38(6), 859-964.

Lamont, M., \& Lareau, A. (1988). Cultural capital: Allusions, gaps and glissandos in recent theoretical developments. Sociological Theory, 6(2), 153-168.

Lee, E. M., \& Kramer, R. (2013). Out with the old, in with the new? Habitus and social mobility at selective colleges. Sociology of Education, 86(1), 18-35.

Light, A., \& Strayer, W. (2000). Determinants of college completion: School quality or student ability? The Journal of Human Resources, 35(2), 299-332.

Longden, B. (2004). Interpreting student early departure from higher education through the lens of cultural capital. Tertiary Education \& Management, 10(2), 121-138.

Lowe, H., \& Cook, A. (2003). Mind the gap: are students prepared for higher education? Journal of Further and Higher Education, 27(1), 53-76.

Lucas, S. R. (2001). Effectively maintained inequality: Education transitions, track mobility, and social background effects. American Journal of Sociology, 106(6), 1642-169o.

Martin, G. L. (2015). "Always in my face": An exploration of social class consciousness, salience, and values. Journal of College Student Development, 56(19), 471-487.

McPherson, M., Smith-Lovin, L., \& Cook, J. M. (2001). Birds of a feather: Homophily in social networks. Annual Review of Sociology, 27, 415-444.

Mayhew, M. J., Rockenbach, A. B., Bowman, N. A., Seifert, T. A., \& Wolniak, G. C. (2016). How college affects students: 21st Century evidence that higher education works. San Francisco: Jossey-Bass.

Miller, G. E., Yu, T., Chen, E., \& Brody, G. H. (2015). Self-control forecasts better psychosocial outcomes but faster epigenetic aging in low-SES youth. Proceedings of the National Academy of Sciences, 112(33), 10325-10330.

Nelson, L. D., Simmons, J., \& Simonsohn, U. (2017). Psychology’s renaissance. Annual Review of Psychology, 69, 511-534.

Nosek, B. A., \& Lakens, D. (2014). Registered reports: A method to increase the credibility of published results. Social Psychology, 45, 137-141. 
Okonofua, J. A., Paunesku, D., \& Walton, G. M. (2016). Brief intervention to encourage empathic discipline cuts suspension rates in half among adolescents. Proceedings of the National Academy of Sciences, 113(19), 5221-5226.

Ovink, S., Kalogrides, D., Nanney, M., \& Delaney, P. (2017). College match and undermatch: Assessing student preferences, college proximity, and inequality in post-college outcomes. Research in Higher Education, 58 (7), 1-38.

Ostrove, J. M., \& Long, S. M. (2007). Social class and belonging: Implications for college adjustment. The Review of Higher Education, 30(4), 363-389.

Padgett, R. D., Johnson, M. P., \& Pascarella, E. T. (2012). First-generation undergraduate students and the impacts of the first year of college: Additional evidence. Journal of College Student Development, 53(2), 243-266.

Parker, P. D., Schoon, I., Tsai, Y. M., Nagy, G., Trautwein, U., \& Eccles, J. S. (2012). Achievement, agency, gender, and socioeconomic background as predictors of postschool choices: A multicontext study. Developmental Psychology, 48(6), 1629-1642.

Pascarella, E. T., Wolniak, G. C., Pierson, C. T., \& Terenzini, P. T. (2003). Experiences and outcomes of first-generation students in community colleges. Journal of College Student Development, 44(3), 420-429.

Radford, A. W. (2013). Top student, top school?: How social class shapes where valedictorians go to college. University of Chicago Press.

Rahe, R. H. (1968). Life-change measurement as a predictor of illness. Proceedings of the Royal Society of Medicine, 61(11), 1124-1126.

Reay, D., Crozier, G., \& Clayton, J. (2010). "Fitting in" or "standing out”: Working-class students in UK higher education. British Educational Research Journal, 36(1), 107-124.

Rodriguez, A. (2015). Tradeoffs and limitations: Understanding the estimation of college undermatch. Research in Higher Education, 56(6), 566-594.

Shavit, Y., \& Blossfeld, H. P. (1993). Persistent inequality: Changing educational attainment in thirteen countries. Social Inequality Series. Westview Press.

Smith, J., Pender, M., \& Howell, J. (2013). The full extent of student-college academic undermatch. Economics of Education Review, 32, 247-261.

Stephens, N. M., Hamedani, M. G., \& Destin, M. (2014). Closing the social-class achievement gap: A difference-education intervention improves first-generation students' academic performance and all students' college transition. Psychological Science, 25, 943-953.

Stephens, N. M., Fryberg, S. A., Markus, H. R., Johnson, C. S., \& Covarrubias, R. (2012). Unseen disadvantage: How American universities' focus on independence undermines the academic performance of first-generation college students. Journal of Personality and Social Psychology,102(6), 1178-1197.

Stephens, N. M., Markus, H. R., \& Fryberg, S. A. (2012). Social class disparities in health and education: Reducing inequality by applying a sociocultural self model of behavior. Psychological Review, 119(4), 723-744.

Stephens, N. M., Townsend, S. S., \& Dittmann, A. G. (2019). Social-class disparities in higher education and professional workplaces: The role of cultural mismatch. Current Directions in Psychological Science, 28(1), 67-73. 
Steptoe, A., Tsuda, A., Tanaka, Y., \& Wardle, J. (2007). Depressive symptoms, socioeconomic background, sense of control, and cultural factors in university students from 23 countries. International Journal of Behavioral Medicine, 14(2), 97-107.

Tieben, N., \& Wolbers, M. H. (2010). Transitions to post-secondary and tertiary education in the Netherlands: A trend analysis of unconditional and conditional socio-economic background effects. Higher Education, 60(1), 85-100.

Wagenmakers, E. J., Wetzels, R., Borsboom, D., van der Maas, H. J. L., \& Kievit, R. A. (2012). An agenda for purely confirmatory research. Perspectives on Psychological Science, 7 , 632-638.

Walpole, M. (2003). Socioeconomic status and college: How SES affects college experiences and outcomes. The Review of Higher Education: Journal of the Association for the Study of Higher Education, 27, 45-73.

Wickrama, K. A. S., O’Neal, C. W., \& Lee, T. K. (2016). The health impact of upward mobility: Does socioeconomic attainment make youth more vulnerable to stressful circumstances? Journal of Youth and Adolescence, 45 (2), 271-285.

Yeager, D. S., Walton, G. M., Brady, S. T., Akcinar, E. N., Paunesku, D., Keane, L., ... Dweck, C. S. (2016). Teaching a lay theory before college narrows achievement gaps at scale. Proceedings of the National Academy of Sciences, 113(24), 3341-3348. 


\subsection{Appendix A}

TABLE A1 Results from Linear Regression Models Predicting Satisfaction among Low-SES Youth in Higher Education (SES definition 2)

\begin{tabular}{|c|c|c|c|c|}
\hline \multirow[b]{2}{*}{ Predictor } & \multicolumn{2}{|c|}{ Model 1 (without covariates) } & \multicolumn{2}{|c|}{ Model 2 (with covariates) } \\
\hline & $b$ & $95 \% \mathrm{Cl}$ & $b$ & $95 \% \mathrm{Cl}$ \\
\hline & \multicolumn{4}{|c|}{ Outcome: satisfaction on content of the study program } \\
\hline Intercept & $8.02^{\star \star}$ & {$[7.91,8.14]$} & $6.31^{\star \star}$ & {$[4.97,7.65]$} \\
\hline Institution (most selective) & 0.02 & {$[-0.11,0.14]$} & -0.03 & {$[-0.15,0.10]$} \\
\hline Average grade final exams & - & - & $0.17^{\star \star}$ & {$[0.09,0.24]$} \\
\hline Age & - & - & 0.03 & {$[-0.03,0.09]$} \\
\hline \multirow[t]{2}{*}{ Gender (female) } & - & - & 0.05 & {$[-0.05,0.14]$} \\
\hline & \multicolumn{4}{|c|}{ Outcome: satisfaction on teaching methods } \\
\hline Intercept & $7.32^{* \star}$ & {$[7.20,7.44]$} & $6.95^{\star *}$ & {$[5.52,8.38]$} \\
\hline Institution (most selective y) & 0.04 & {$[-0.09,0.18]$} & 0.02 & {$[-0.12,0.15]$} \\
\hline Average grade final exams & - & - & $0.09^{*}$ & {$[0.01,0.17]$} \\
\hline Age & - & - & -0.01 & {$[-0.08,0.05]$} \\
\hline \multirow[t]{2}{*}{ Gender (female) } & - & - & 0.03 & {$[-0.07,0.13]$} \\
\hline & \multicolumn{4}{|c|}{ Outcome: satisfaction on quality of lecturers } \\
\hline Intercept & $7.31^{\star \star}$ & {$[7.18,7.44]$} & $5.57^{\star \star}$ & {$[4.07,7.08]$} \\
\hline Institution (most selective) & 0.11 & {$[-0.03,0.25]$} & 0.06 & {$[-0.08,0.20]$} \\
\hline Average grade final exams & - & - & $0.13^{\star \star}$ & {$[0.05,0.22]$} \\
\hline Age & - & - & 0.05 & {$[-0.02,0.12]$} \\
\hline \multirow[t]{2}{*}{ Gender (female) } & - & - & -0.09 & {$[-0.20,0.02]$} \\
\hline & \multicolumn{4}{|c|}{ Outcome: satisfaction on personal contact with lecturers } \\
\hline Intercept & $7.70^{\star \star}$ & {$[7.51,7.89]$} & $4.21^{\star \star}$ & {$[1.95,6.48]$} \\
\hline Institution (most selective) & $-1.13^{\star \star}$ & {$[-1.34,-0.92]$} & $-1.21^{\star \star}$ & {$[-1.42,-1.00]$} \\
\hline Average grade final exams & - & - & $0.25^{* *}$ & {$[0.12,0.37]$} \\
\hline Age & $-0.16^{*}$ & {$[-0.32,0.00]$} & $0.11^{*}$ & {$[0.00,0.21]$} \\
\hline \multirow[t]{2}{*}{ Gender (female) } & - & - & $-0.16^{\star}$ & {$[-0.32,0.00]$} \\
\hline & \multicolumn{4}{|c|}{ Outcome: satisfaction on personal contact with fellow students } \\
\hline Intercept & $8.33^{\star \star}$ & {$[8.18,8.48]$} & $8.40^{\star \star}$ & {$[6.62,10.17]$} \\
\hline Institution (most selective) & $-0.49^{\star \star}$ & {$[-0.66,-0.33]$} & $-0.53^{\star \star}$ & {$[-0.69,-0.36]$} \\
\hline Average grade final exams & - & - & $0.10^{\star}$ & {$[0.00,0.19]$} \\
\hline Age & - & - & -0.04 & {$[-0.12,0.04]$} \\
\hline Gender (female) & - & - & 0.03 & {$[-0.10,0.15]$} \\
\hline
\end{tabular}

Note. $\mathrm{Cl}=$ confidence interval, ${ }^{\star} p<.05,{ }^{\star \star} p<.01$. 
TABLE A2 Results from Linear Regression Models Predicting Satisfaction among Students from All SES-Backgrounds in Higher Education (SES definition 2)

\begin{tabular}{|c|c|c|c|c|}
\hline \multirow[b]{2}{*}{ Predictor } & \multicolumn{2}{|c|}{ Model 1 (without covariates) } & \multicolumn{2}{|c|}{ Model 2 (with covariates) } \\
\hline & $b$ & $95 \% \mathrm{Cl}$ & $b$ & $95 \% \mathrm{Cl}$ \\
\hline & \multicolumn{4}{|c|}{ Outcome: satisfaction on content of the study program } \\
\hline Intercept & $7.90^{\star \star}$ & {$[7.81,7.99]$} & $6.64^{\star \star}$ & {$[5.87,7.40]$} \\
\hline Institution (most selective) & $0.21^{\star \star}$ & {$[0.11,0.31]$} & $0.12^{*}$ & {$[0.02,0.22]$} \\
\hline SES (low-SES) & 0.12 & {$[-0.02,0.27]$} & 0.12 & {$[-0.02,0.27]$} \\
\hline Institution x SES & $-0.19^{\star}$ & {$[-0.35,-0.03]$} & -0.15 & {$[-0.31,0.01]$} \\
\hline Average grade final exams & - & - & $0.16^{\star \star}$ & {$[0.13,0.20]$} \\
\hline Age & - & - & 0.01 & {$[-0.03,0.04]$} \\
\hline \multirow[t]{2}{*}{ Gender (female) } & - & - & $0.06^{*}$ & {$[0.01,0.11]$} \\
\hline & \multicolumn{4}{|c|}{ Outcome: satisfaction on teaching methods } \\
\hline Intercept & $7.19^{\star \star}$ & {$[7.09,7.30]$} & $6.64^{\star *}$ & {$[5.79,7.49]$} \\
\hline Institution (most selective) & $0.21^{\star \star}$ & {$[0.10,0.32]$} & $0.14^{\star \star}$ & {$[0.03,0.25]$} \\
\hline SES (low-SES) & 0.13 & {$[-0.04,0.29]$} & 0.13 & {$[-0.04,0.29]$} \\
\hline Institution x SES & -0.16 & {$[-0.34,0.01]$} & -0.13 & {$[-0.31,0.04]$} \\
\hline Average grade final exams & - & - & $0.12^{\star \star}$ & {$[0.08,0.16]$} \\
\hline Age & - & - & -0.01 & {$[-0.05,0.03]$} \\
\hline \multirow[t]{2}{*}{ Gender (female) } & - & - & 0.04 & {$[-0.01,0.10]$} \\
\hline & \multicolumn{4}{|c|}{ Outcome: satisfaction on quality of lecturers } \\
\hline Intercept & $7.20^{\star \star}$ & {$[7.10,7.31]$} & $5.46^{\star \star}$ & {$[4.61,6.31]$} \\
\hline Institution (most selective) & $0.24^{\star *}$ & {$[0.13,0.35]$} & $0.15^{\star \star}$ & {$[0.04,0.26]$} \\
\hline SES (low-SES) & 0.11 & {$[-0.06,0.27]$} & 0.11 & {$[-0.06,0.27]$} \\
\hline Institution x SES & -0.13 & {$[-0.31,0.05]$} & -0.10 & {$[-0.27,0.08]$} \\
\hline Average grade final exams & - & - & $0.16^{\star *}$ & {$[0.12,0.20]$} \\
\hline Age & - & - & 0.04 & {$[0.00,0.08]$} \\
\hline \multirow[t]{2}{*}{ Gender (female) } & - & - & -0.04 & {$[-0.10,0.01]$} \\
\hline & \multicolumn{4}{|c|}{ Outcome: satisfaction on personal contact with lecturers } \\
\hline Intercept & $7.58^{\star \star}$ & {$[7.42,7.74]$} & $4.82^{\star \star}$ & {$[3.53,6.11]$} \\
\hline Institution (most selective) & $-0.91^{\star \star}$ & {$[-1.07,-0.74]$} & $-1.06^{\star \star}$ & {$[-1.23,-0.89]$} \\
\hline SES (low-SES) & 0.12 & {$[-0.13,0.37]$} & 0.12 & {$[-0.13,0.37]$} \\
\hline Institution x SES & -0.22 & {$[-0.49,0.05]$} & -0.16 & {$[-0.43,0.11]$} \\
\hline Average grade final exams & - & - & $0.27^{\star \star}$ & {$[0.21,0.34]$} \\
\hline Age & - & - & 0.06 & {$[0.00,0.12]$} \\
\hline \multirow[t]{2}{*}{ Gender (female) } & - & - & $-0.12^{\star \star}$ & {$[-0.21,-0.04]$} \\
\hline & \multicolumn{4}{|c|}{ Outcome: satisfaction on personal contact with fellow students } \\
\hline Intercept & $8.20^{\star \star}$ & {$[8.08,8.32]$} & $8.23^{\star \star}$ & {$[7.24,9.22]$} \\
\hline Institution (most selective) & $-0.23^{\star \star}$ & {$[-0.36,-0.11]$} & $-0.31^{\star \star}$ & {$[-0.44,-0.18]$} \\
\hline SES (low-SES) & 0.13 & {$[-0.06,0.32]$} & 0.13 & {$[-0.06,0.32]$} \\
\hline Institution x SES & $-0.26^{*}$ & {$[-0.47,-0.06]$} & $-0.23^{*}$ & {$[-0.44,-0.02]$} \\
\hline Average grade final exams & - & & $0.12^{\star \star}$ & {$[0.07,0.17]$} \\
\hline Age & - & & -0.05 & {$[-0.09,0.00]$} \\
\hline Gender (female) & - & & 0.02 & {$[-0.05,0.09]$} \\
\hline
\end{tabular}




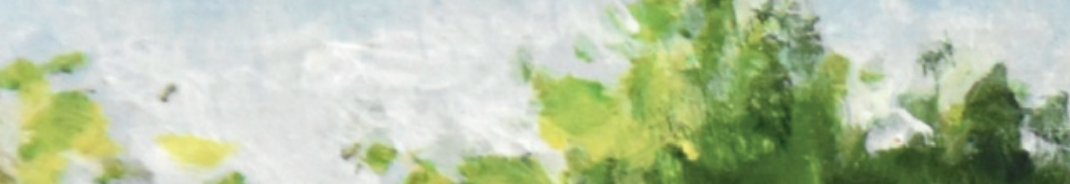

tes is un wat

$2 x-4 x^{2}$

\section{$2=$ \\ SW \\ $x_{2} \cos ^{2}$ \\ $8 x$ \\ $\approx$}

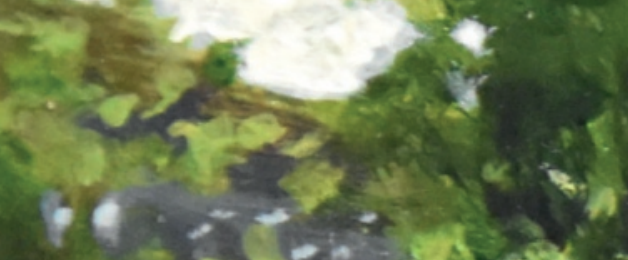

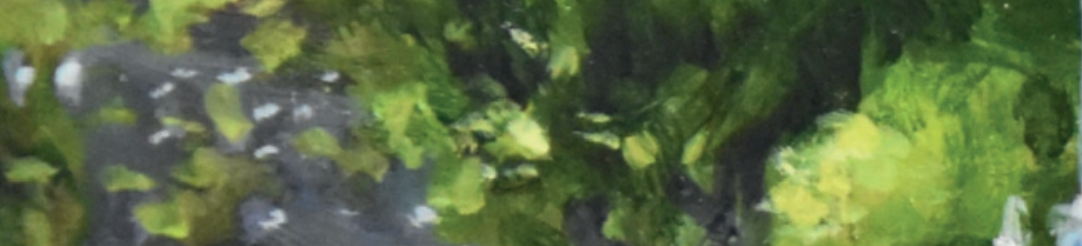

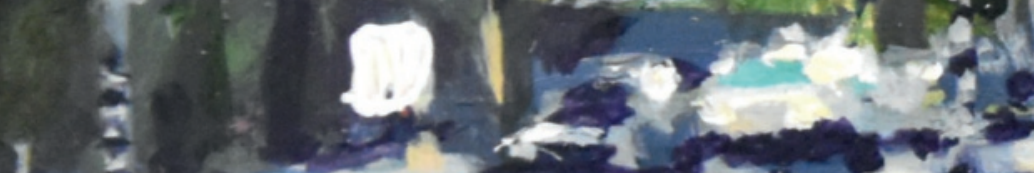

$1 \frac{2}{3}=2$

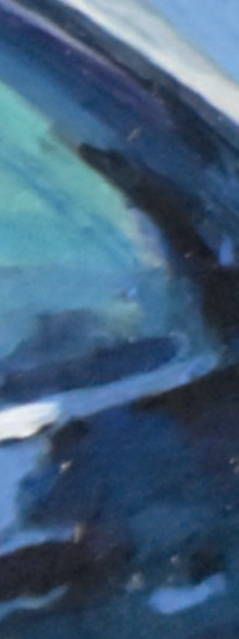




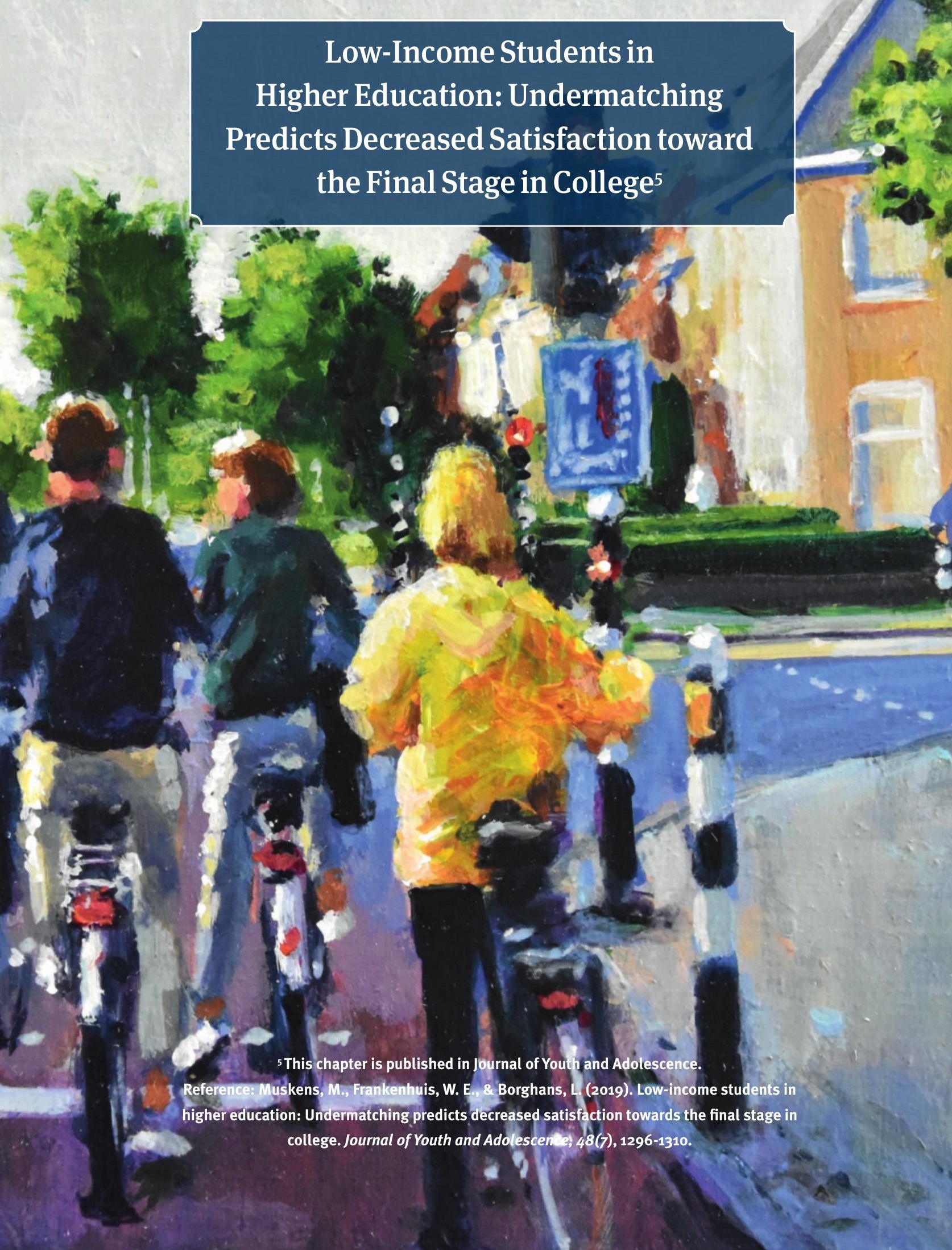




\begin{abstract}
It is undesirable when students attend institutions that are less selective than their academic credentials would permit (i.e., undermatching) because of the long-term consequences for their job opportunities and wages, in particular for students from lowsocioeconomic (SES) backgrounds. Undermatching may also affect students' satisfaction during college. Research from a life course perspective shows that subjective experiences during college may have long-term impact on adolescents' development. However, little is known about the relation between undermatching and students' subjective experiences during their years in college, and about whether this relation is moderated by SES. From an academic misalignment perspective, undermatching may lead to less satisfaction because undermatched students are not maximizing their potential. However, from a social misalignment perspective, experiences of social mismatch when low-SES students enter the most selective institutions are well documented, and such mismatch may be less pronounced in less selective institutions. Consequently, there may be a positive relation between undermatching and satisfaction with the social environment for low-SES students. The current study tested these relations by using propensity score matching (PSM) to analyse the association between undermatching, SES, and satisfaction among 21,452 respondents $\left(67 \%\right.$ female) among $1^{\text {st }}, 2^{\text {nd }}, 3^{\text {th }}$, and $4^{\text {th }}$ year college students from a cohort study among students in the Netherlands (Dutch Student Monitor), all of whom were eligible for the most selective institutions. The results indicated a negative relation between undermatching and satisfaction with the social and academic environment, both for low- and high-SES students. The negative relation between undermatching and both forms of satisfaction increases toward the last year in college, especially for low-SES students. This lowered satisfaction in the final stage in higher education implies that the negative consequences of undermatching become more pronounced after students have become more integrated in their colleges. These findings have important implications for the understanding about undermatching in relation to students' development and for the formulation of policies and programs for promoting social mobility.
\end{abstract}




\subsection{Introduction}

Graduating from the most selective institutions is related to more career opportunities and higher wages in the long term compared to graduating from less selective institutions (Mayhew, Rockenbach, Bowman, Seifert, \& Wolniak, 2016). Because of these economic benefits of college completion in selective institutions, undermatching, when students attend institutions that are less selective than their academic achievements would enable, is an undesirable outcome (Tiboris, 2014). In addition, from an academic misalignment perspective, undermatching leads to less positive college experiences than matching. Because of the less rigorous study program in less selective institutions, high-achieving undermatched students would be less challenged to develop their full potential (Hoxby \& Turner, 2013). This mismatch may lead to less academic integration, contributing to the risk of dropping out of college (Bowen, Chingos, \& McPherson, 2009). The recurrent finding that undermatching is negatively related to college completion (Ovink, Kalogrides, Nanney, \& Delaney, 2018) supports this hypothesis. Importantly, not all groups are equally likely to undermatch. Social scientists and policymakers are particularly worried about the high prevalence of undermatching among students from low-socioeconomic (low-SES) backgrounds (Howell \& Pender, 2016), because this leads to reinforcement of social and economic inequality (Deutschlander, 2017).

Research from a life course perspective has shown that early circumstances and experiences can have cumulative and enduring consequences for life experiences in later developmental stages (Yoshioka \& Noguchi, 2009). More specifically, adolescents' subjective experiences during their years in college may have far-reaching consequences for their development toward adulthood, for example for academic achievement and psychosocial well-being (Wickrama, O’Neal, \& Lee, 2016). However, little is known about the subjective experiences of students who undermatch. The present study will investigate the relationship between undermatching, socioeconomic status, and students' subjective experiences.

\section{Socioeconomic Status}

An extensive body of research has shown that students' SES is one of the strongest correlates of academic performance (e.g., Sirin, 2005). In addition, the size of the relationship between SES and educational attainment increases by each school level, suggesting that the gap between low- and high-SES students in academic achievement expands through students' lives (Sirin, 2005). The relation between SES and educational achievement is related to several factors, such as differences in cognitive development related to harmful effects of poverty (Mullainathan \& Sharif, 2013), low-SES students' higher chance to encounter lower educational expectations from their parents (DavisKean, 2005), and their teachers (Jussim, Eccles, \& Madon, 1996), and a higher likelihood of being placed in low-resource schools compared to high-SES students (Lee \& Burkam, 2002). Moreover, researchers have also suggested that there may be a mismatch between the social-cognitive skills that children from disadvantaged circumstances develop in 
interaction with their home environments, and social-cognitive skills demanded in schoolenvironments (Ellis, Bianchi, Griskevicius, \& Frankenhuis, 2017). For example, in higher education, a cultural mismatch with regard to norms in interacting with others seems to fuel social-class disparities (Stephens, Townsend, \& Dittmann, 2018).

\section{Student experiences}

Theoretical models of student experiences in college distinguish between integration in the social and academic system. In the classical work of Tinto (1975), integration in these two systems was defined as primary condition for student success. The social environment refers to social aspects of college, such as interactions with fellow students and teachers, whereas the academic environment refers to intellectual aspects of college, such as the content and structure of the study program. Indeed, decades of research testing Tinto's model in different college systems and environments, has shown that when examining students' integration, it is valuable to distinguish between the social and academic environment (Aljohani, 2016).

Notably, theories about cultural capital predict difficulties with integration in the social environment when low-SES youth enter the most selective institutions, and the existence of these social barriers is well documented (Jury et al., 2017). Differences in cultural capital and codes between low- and high-SES students (Jæger, 2009), signalled by teachers and fellow-students, might lead to social exclusion of low-SES students (Walpole, 2003). This experience of not fitting in can lead to lower levels of satisfaction with the social environment in the most selective tracks (Jury et al., 2017). This mismatch may be less present in less selective institutions because of the presence of more low-SES students (Bastedo \& Jaquette, 2011), and, as a consequence, cultural codes that may match better with low-SES students' backgrounds (Walpole, 2003). At the same time, it is also suggested that undermatching leads to a misalignment between students' academic capacities and demands from the educational program. This misalignment may lead to less satisfaction with academic aspects in college (Hoxby \& Turner, 2013), and difficulties with integration in the academic environment (Aljohani, 2016). By contrast, in the most selective tracks, highly qualified students may be more likely to experience an academic match (Hoxby \& Avery, 2013).

Student integration is a developmental process, and classical research on students' integration suggests that students go through several stages after their transitions into college. First, there is the stage of separation when students start to detach themselves from the norms and values of their previous communities, such as family and high school. Second, students go through the phase of transition, when they adapt new norms and value from their college. Third, in the phase of incorporation, students are successfully integrating in the new community (Aljohani, 2016). Therefore, in studying student experiences, it is important to incorporate a time perspective. 


\section{Undermatching in the Netherlands}

Research on the phenomenon of undermatching has been conducted mainly in the educational context of the U.S. To determine undermatching, researchers have to define institutions' selectivity levels and, in addition, they have to determine which students are eligible to gain admission to these levels. These distinctions are in practice gradual. Because researchers have used different approaches in defining both elements, estimating undermatching has leaded to different results, such as a wide range of undermatch rates, and conflicting findings for underrepresented students (Rodriguez, 2015). By focussing on undermatching in the Netherlands, these issues are circumvented, since the Dutch education system has a clear distinction between two levels of higher education, with almost no variation in quality within each level, and there are clear rules who can attend the higher level. On other aspects, the Dutch situation is very similar to higher education in the U.S. and in Europe. Both in the U.S. and in Europe, high school (or secondary education) is followed by postsecondary (or tertiary) education, generally during late adolescence. The contexts are similar regarding academic degrees that can be attained, and educational and professional careers during and after higher education. In addition, patterns of educational inequality, such as the tendency among low-SES students to undermatch (Dutch Inspectorate of Education, 2018), are comparable between the U.S and the European context.

The main differences the current study takes advantage of are differences in admission procedures and institutions selectivity. First, there are differences with regard to admission procedures to higher education. In the U.S, institutions for higher education determine their own admission standards, and therefore, it depends on the specific higher education institution who is admitted and who is not. Students' eligibility is determined in the last stage of high school, with performance on standardized tests (i.e., SAT, ACT), and by assessing their academic and extracurricular performance. In the Netherlands, as in many other European countries, students' eligibility for higher education is determined by students' track in secondary education, and all institutions of higher education follow these standards. In the Netherlands, the eligibility for the most selective institutions is determined by having completed the highest secondary track (i.e., VWO), whereas participating in a lower track (i.e., HAVO) leads to eligibility to less selective institutions. Therefore, in the Netherlands, undermatching is a consequence of students' choice to attend a less selective institution after completing the highest secondary track. The prevalence of undermatching among students with the eligibility to attend the most selective institutions ranges between $10 \%$ and $13 \%$ (Van den Broek et al., 2018).

Second, there is an important difference between the U.S. context and the European context regarding higher education institutions' selectivity. Whereas in the U.S. context, selectivity is often seen as a continuum (Roderick et al., 2006), in the Dutch context there are only two types of higher education institutions: most selective institutions and less selective institutions. In the U.S. context, both students' admissibility and institutions' selectivity have to be empirically estimated, leading to discussion about the accuracy of 
the estimation of undermatching (Smith, Pender, \& Howell, 2013). By contrast, in the Dutch context, both students' admissibility and institutions' selectivity are fully transparent and clearly defined, and therefore undermatching is easy to determine. Respondents are determined as 'matched' when they are eligible for the most selective institutions and subsequently enrolled in an institution that can be characterized as 'most selective'. Respondents are determined as 'undermatched' when they are eligible for the most selective institutions and subsequently have chosen to enrol in an institution that is less selective.

\section{Current Study}

The predominant view is that undermatching is undesirable and has negative longterm consequences for wages and job status. Subjective experiences during college are also important because of their relation with college completion and, from a life course development perspective, because these experiences accumulate and may have enduring consequences during adulthood. However, little is known about the relation between undermatching and subjective experiences of students during their years in higher education, and about the possibility that students' SES plays a moderating role in this relation. Using a representative sample of 22,521 adolescents, the current study attempts to enhance knowledge about these relations and their development over the course of students' time in higher education. From an academic misalignment perspective, undermatching leads to less satisfaction because of the mismatch between students' high capacity and the less rigorous educational program in less selective institutions. However, from a social misalignment perspective, undermatching may have differential effects for low- and high-SES students, predicting more satisfaction for students with a low-SES background because of less experiences of social mismatch in the less selective institutions than in the highly selective institutions. In addition, the role of students' time spent in college (i.e., $1^{\text {st }}, 2^{\text {nd }}, 3^{\text {th }}$ and $4^{\text {th }}$ year) in these relationships is explored.

\subsection{Methods}

\section{Participants and Data Set}

Research questions, inclusion criteria, sample size, and constructs of this study are preregistered (Frankenhuis \& Nettle, 2018) at Open Science Framework (https://osf. io/4x5p8/). During the review procedure, this study has deviated from the preregistration on several aspects, reported in an addendum added to the preregistration (https://osf.io/ crhjx/). This section reports how the sample size was determined, all data exclusions, and all measures in the study.

Data for this study come from the Dutch Student Monitor, a large-scale survey of youth's experiences during their time in higher education in the Netherlands. Participants were selected with a random sample procedure from all higher education institutions in the 
Netherlands. The dataset used for this study contains data from 3 waves (from 2013 to 2015; $N=58,177$ ). To minimize pre-existing differences in skills and cognitive abilities, only respondents were included who are eligible for the most selective institutions (i.e., about $45 \%$ of this sample), who are enrolled in higher education (i.e., university or higher professional education) in a full-time educational program, and who are between 17 and 25 years old $(M=21.58, S D=1.77)$. Therefore, the final sample includes 21,452 students who are all eligible for the most selective institutions. The majority (87\%) chose to enrol in the most selective institutions (i.e., matched students), and a minority (13\%) chose to enrol in less selective institutions (i.e., undermatched students).

\section{Measures}

Satisfaction. The outcome measure of interest is student satisfaction. Because of the theoretical model for student integration that this study builds on (Davidson \& Wilson, 2013), two scales for satisfaction were applied: (1) satisfaction with the social environment, and (2) satisfaction with the academic environment. In the Student Monitor survey, participants responded to 20 single items on satisfaction. From these items, drawing on this theoretical model, three items were selected, that reflect their experiences with the social environment, and three items were selected that capture their experiences with the academic environment. Students responded using a 5 -point Likert scale $(1=$ very dissatisfied, 5 = very satisfied) assessing how satisfied they are with several aspects regarding their experiences in college. The scale for social satisfaction was constructed from the items regarding satisfaction with: (1) the general atmosphere, (2) students' attitude toward fellow students, and (3) teachers' attitude toward students. The scale for academic satisfaction was constructed from the items regarding satisfaction with: (1) the content of the programme, (2) general skills learned in the programme, and (3) the degree to which the educational program is academically challenging. The two scales were reidentified by a joint factor analysis, explaining $65 \%$ of the variance. Internal consistencies (Cronbach's Alpha) were .72 for both scales.

Match and Undermatch. The treatment variable is undermatching. In the Netherlands, students are either eligible for the most selective institutions of higher education (i.e., they attained a diploma in the highest level in high school) or not (i.e., they have not attained a diploma in the highest level in high school ${ }^{6}$ ). In addition, there are two types of institutions in higher education: most selective institutions (i.e., students are only admitted when they have attained a diploma in the highest level of high school 7 ), and less selective institutions (i.e., a diploma from the highest level of secondary education (i.e., vWO) is not

\footnotetext{
${ }^{6}$ Although the vast majority of students in the most selective institutions in higher education are admitted after attainment of a diploma in the highest level of secondary education, a bachelors' degree attained in a less selective institution in higher education can also give eligibility to the most selective institutions in higher education (applies to less than $4 \%$ off all students in the most selective institutions) (van den Broek et.al., 2018).

${ }^{7}$ Apart from some exceptions regarding specific majors, such as dentistry and medicine, selective institutions hold no additional requirements.
} 
required, a diploma in a lower level of secondary education (i.e., HAVO) gives eligibility). Respondents were determined as 'matched' when they are eligible for the most selective institutions in higher education and subsequently choose to attend an institution that can be characterized as 'most selective'. Respondents are determined as 'undermatched' when they are eligible for the most selective institutions in higher education and subsequently have chosen to attend an institution that is less selective.

Socioeconomic Status. SES was measured in two ways. Because various components of SES could work through different processes when affecting health and psychosocial outcomes, using more than one single measure of SES is important. Following recommendations by the APA task force Socioeconomic Status (2007), an objective and a subjective measure of SES was employed. Research has shown that subjective social status is correlated with well-established measures of SES, but also that it may capture unique aspects socioeconomic circumstances that predict outcomes related to wellbeing often missed by objective indicators of SES (Bradshaw, Kent, Henderson, \& Setar, 2017). This study thus included: 1 ) an objective measure based on parental educational level with two levels: (1, Low-SES), both parents have not obtained a diploma in higher education, (o, High-SES), one or both parents obtained a diploma in higher education, and 2) a subjective measure (Singh-Manoux, Adler, \& Marmot, 2003) based on a single item regarding students' view of their parents' social class ("Some people belong to a higher social class, others to a lower one. Considering your own social background, where on the scale would you place your parents/caregivers?"). The response scale ranged from 1, lower social class, to 10 , higher social class.

\section{Covariates}

Grades. In Dutch education, the grading system consists of marks from 1 (very poor) to 10 (outstanding). The pass mark for a single course is ' 6 '. Students' average grades were measured by asking them to report their average grade during their last year in high school using a single-item question. Self-reported grades are reasonably good reflections of actual grades, especially among high-performers, and are strong predictors of future grade points (Kuncel, Credé, \& Thomas, 2005).

Motivation Before College. To control for the potential confounding effects of differences in students' motivation before their transition to higher education, respondents were asked how motivated they were before going to college to attain a degree in higher education on a 5 -point Likert scale $(1=$ not at all motivated, 5 = very motivated).

Additional Controls. Other covariates in the models include indicators for gender, age, immigrant status (i.e., at least one parent was born abroad) (with non-immigrant status as reference category), the language spoken at home with parents (with Dutch as reference category), students' disability (measured with a question about suffering from a disability or chronicle disease (yes $=1$, no $=0$ ), and college major. Descriptive statistics for all variables are displayed in Table 1. 


\section{Analytic Plan}

In testing the relation between undermatching in student satisfaction, selection effects might play a role. Students who choose to undermatch may differ from students who choose to go the most selective institutions on several characteristics. For example, it is possible that undermatched students, compared to matched students, are likely to be less motivated to attain a degree before their entrance to college. In this way, matched and undermatched students differ on salient characteristics that spuriously inflate the effect that undermatching would have on the development of satisfaction when using traditional methodological techniques. For that reason, propensity score matching (PMS; Thoemmes \& Kim, 2011) was applied. PSM creates a treatment group (in the current study, students who are undermatched) and a comparison group (in the current study, students who are matched), and compares these groups on the outcome measure of interest (in the current study, student satisfaction). PSM creates two equal groups by matching them on several covariates that may affect the propensity to undermatch and student satisfaction (Rosenbaum \& Rubin, 1983). More specifically, PSM estimates a logit model predicting the treatment (i.e., undermatching) with covariates. In this study, the propensity to undermatch was first estimated with a logit model (conducted with $R$ essentials for SPSS 24) that predicts undermatch with the covariates described earlier (except for the covariate college major, because experiences with college major occurred after being matched or undermatched). Each respondent was assigned a propensity to undermatch, varying from propensity scores close to o (very low chance to undermatch) to 1 (very high chance to undermatch). Second, respondents from the treatment group (i.e., undermatched students) were matched with respondents from the comparison group (i.e., matched students) using the propensity scores. In the analyses presented, the two-toone nearest-neighbour matching technique was applied, without replacement and with a conservative .02 caliper level. The two-to-one nearest-neighbour matching technique was the most suitable approach because there are more untreated (matched) respondents ( $N$ $=18,626)$ than treated (undermatched) respondents $(\mathrm{N}=2,826)$ in the dataset, and the 2:1 ratio is found to improve precision without a commensurate increase in bias (Austin, 2010). (However, estimating models with the specification one-to-one nearest neighbour matching appeared to show the same results as the two-to-one nearest neighbour matching, results not shown). Each undermatched student was matched to a maximum of two matched student based on their propensity score, and the difference between these two respondents was not larger than 0.02 . This resulted in 2,826 students in the treatment and 5,507 respondents in the comparison group.

After following the PSM procedure, the relation between undermatching, SES, and satisfaction was tested with linear regression models, applying satisfaction (with the social and academic environment, separate analyses) as dependent variable, undermatching, SES, and the interaction undermatching $\times$ SES as predictors, and college major as covariate (eight dummy variables). In addition, in order to assess the development of these relationships during the years in college, the predictor year, and the interaction undermatching $\times$ SES $\times$ year (and all relevant two-way interactions) were added to the 
model. Finally, the simple mean differences with regard to year, SES and undermatching were evaluated. The presentation of the results is focuses on results of interactions in linear regression models (conducted with software package SPSS 24), as these assess the research questions.

\section{$5 \cdot 3$ Results}

Descriptive statistics and correlations among study variables are shown in Table 1.

The analysis began by estimating a logit model by predicting undermatching with nine covariates. Most of the covariates were statistically significant predictors of undermatching (see Table 2).

Overall, five of the covariates reached statistical significance ( $p<.01$, two-tailed tests). Covariates that were not a significant predictor of undermatching, such as age, were still included in the final model to create the propensity score.

Next, a two-to-one nearest-neighbour matching technique was conducted without replacement and with a .02 caliper level (i.e., matched neighbours differ no more than .02 standard deviations of the logit of the estimated propensity score). Results indicate that the matching was successful, with all covariates having a standardized mean difference smaller than .25 after matching. In addition, the Relative Multivariate Imbalance test showed a lower L1 after matching (.670) than before matching (.732) (lacus, King, \& Porro, 2011), indicating that the matching procedure generated balance.

Table 3 shows the results of the pre- and postmatching t-tests, examining differences between undermatched and matched students on the covariates. In the left-hand columns (i.e., the unmatched sample), can be seen that matched and undermatched students differ significantly on six out of nine covariates. These significant differences show that the results may be confounded when analysing the relationship between undermatching and students' satisfaction, and that PSM may address this issue. In the right-hand columns (i.e., the matched sample), the results are shown of t-tests after matching on the propensity score. The results show that matching was indeed successful, because it eliminated all significant differences on covariates between matched and undermatched students.

Because the PSM procedure generated balance between undermatched and matched students with regard to covariates, next, the matched dataset was appropriate for examining the interaction between undermatching and SES in relation to student satisfaction. The results of the final analyses, with satisfaction as outcome measure, undermatching, SES, and the interaction undermatching $\times$ SES as predictor, and college major as covariates, are shown in Table 4. The results show no interaction between undermatching and SES. 
TABLE 1 Descriptive Statistics among Study Variables

\begin{tabular}{|c|c|c|c|c|c|c|c|c|c|c|c|}
\hline & 1 & 2 & 3 & 4 & 5 & 6 & 7 & 8 & 9 & 10 & 11 \\
\hline \multicolumn{12}{|l|}{1 Satisfaction (social) } \\
\hline 2 Satisfaction (academic) environment & $0.47^{\star}$ & & & & & & & & & & \\
\hline 3 Undermatched & $-0.07^{\star}$ & $-0.10^{\star}$ & & & & & & & & & \\
\hline 4 SES def. 1 & $-0.03^{*}$ & $-0.03^{*}$ & $0.08^{*}$ & & & & & & & & \\
\hline 5 SES def. 2 & $0.05^{*}$ & $0.06^{\star}$ & $-0.04^{*}$ & $-0.41^{*}$ & & & & & & & \\
\hline 6 Male & $0.02^{*}$ & 0.01 & $-0.05^{*}$ & 0.01 & $-0.03^{*}$ & & & & & & \\
\hline 7 Age & $-0.05^{*}$ & $-0.04^{*}$ & 0.00 & $0.02^{*}$ & 0.01 & $0.07^{\star}$ & & & & & \\
\hline 8 Immigrant status & $-0.07^{\star}$ & $-0.02^{*}$ & $-0.03^{*}$ & $0.05^{\star}$ & $-0.11^{*}$ & $0.02^{*}$ & $0.03^{*}$ & & & & \\
\hline 9 Home language Dutch & $0.06^{*}$ & $0.02^{\star}$ & $0.01^{*}$ & $-0.05^{*}$ & $0.10^{*}$ & -0.01 & $-0.03^{*}$ & $-0.35^{*}$ & & & \\
\hline 10 No disability / disease & $0.03^{*}$ & $0.02^{\star}$ & $-0.02^{*}$ & -0.01 & $0.05^{*}$ & $0.07^{\star}$ & $-0.03^{*}$ & 0.00 & 0.00 & & \\
\hline 11 Grade in high school secondary education & $0.08^{*}$ & $0.08^{*}$ & $-0.22^{*}$ & $-0.12^{*}$ & $0.08^{*}$ & $0.02^{\star}$ & 0.00 & $-0.02^{\star}$ & $-0.03^{*}$ & $0.05^{\star}$ & \\
\hline 12 Motivation before college & $0.16^{\star}$ & $0.17^{*}$ & $0.04^{*}$ & 0.00 & $0.03^{*}$ & $-0.09^{*}$ & $-0.03^{*}$ & -0.01 & 0.00 & -0.01 & $0.09^{\star}$ \\
\hline 13 Years in higher education & -0.01 & $-0.04^{\star}$ & $-0.07^{\star}$ & 0.01 & $0.01^{*}$ & $0.03^{\star}$ & $0.78^{\star}$ & $-0.02^{*}$ & 0.00 & $0.03^{*}$ & $0.12^{*}$ \\
\hline 14 Economics & $-0.06^{*}$ & $-0.05^{*}$ & $0.12^{\star}$ & $0.03^{*}$ & 0.01 & $0.08^{*}$ & -0.05 & $0.03^{*}$ & $-0.04^{*}$ & $0.04^{\star}$ & $-0.02^{*}$ \\
\hline 15 Education & 0.01 & $-0.02^{\star}$ & $0.27^{\star}$ & 0.01 & 0.00 & $-0.05^{*}$ & $0.00^{*}$ & $-0.02^{*}$ & 0.01 & $-0.01^{*}$ & $-0.06^{\star}$ \\
\hline 16 Agriculture & $0.11^{\star}$ & $0.04^{\star}$ & $-0.05^{*}$ & $-0.01^{*}$ & 0.00 & $-0.03^{*}$ & $-0.02^{*}$ & $-0.04^{*}$ & $0.01^{*}$ & $-0.02^{*}$ & $-0.04^{*}$ \\
\hline 17 Nature & $0.06^{*}$ & $0.04^{*}$ & $-0.12^{*}$ & $-0.03^{*}$ & 0.00 & $0.08^{*}$ & 0.00 & $0.02^{*}$ & 0.00 & $-0.01^{*}$ & $0.09^{*}$ \\
\hline 18 Science & 0.01 & $0.03^{*}$ & $0.02^{*}$ & -0.01 & 0.00 & $0.28^{\star}$ & 0.01 & $-0.02^{*}$ & $0.02^{\star}$ & $0.01^{*}$ & $0.04^{*}$ \\
\hline 19 Health & $-0.05^{*}$ & $0.04^{*}$ & $0.01^{\star}$ & $-0.02^{*}$ & $0.05^{*}$ & $-0.15^{*}$ & $0.06^{*}$ & $0.02^{*}$ & 0.00 & $0.02^{\star}$ & $0.07^{\star}$ \\
\hline 20 Law & $-0.06^{*}$ & $-0.02^{*}$ & $-0.07^{\star}$ & $0.03^{\star}$ & $0.00^{*}$ & $-0.02^{*}$ & -0.01 & $0.03^{*}$ & $-0.03^{*}$ & $-0.01^{\star}$ & $-0.03^{*}$ \\
\hline 21 Behavior & $-0.04^{*}$ & $-0.08^{*}$ & $-0.03^{\star}$ & $0.02^{\star}$ & $-0.03^{*}$ & $-0.14^{*}$ & -0.01 & 0.00 & $0.02^{\star}$ & $-0.01^{*}$ & $-0.14^{*}$ \\
\hline 22 Language & $0.05^{\star}$ & 0.01 & $-0.03^{*}$ & 0.00 & -0.03 & $-0.07^{\star}$ & 0.01 & $-0.03^{*}$ & 0.00 & $-0.04^{*}$ & $0.03^{*}$ \\
\hline Mean or Proportion & 4.10 & 3.85 & 0.13 & 0.31 & 6.78 & 0.33 & 21.58 & 0.06 & 0.92 & 0.72 & 7.21 \\
\hline SD & 0.61 & 0.64 & 0.34 & 0.46 & 1.50 & 0.47 & 1.77 & 0.23 & 0.27 & 0.45 & 0.64 \\
\hline Minimum & 1 & 1 & 0 & 0 & 1 & 0 & 17 & -1 & 0 & 0 & 5.51 \\
\hline \multirow[t]{2}{*}{ Maximum } & 5 & 5 & 1 & 1 & 10 & 1 & 25 & 1 & 1 & 1 & 10 \\
\hline & 12 & 13 & 14 & 15 & 16 & 17 & 18 & 19 & 20 & 21 & 22 \\
\hline \multicolumn{12}{|l|}{1 Satisfaction (social) } \\
\hline \multirow{2}{*}{\multicolumn{12}{|c|}{$\begin{array}{l}2 \text { Satisfaction (academic) environment } \\
3 \text { Undermatched }\end{array}$}} \\
\hline & & & & & & & & & & & \\
\hline \multicolumn{12}{|l|}{4 SES def. 1} \\
\hline \multicolumn{12}{|l|}{5 SES def. 2} \\
\hline \multicolumn{12}{|l|}{6 Male } \\
\hline \multicolumn{12}{|l|}{7 Age } \\
\hline \multicolumn{12}{|l|}{8 Immigrant status } \\
\hline \multicolumn{12}{|l|}{9 Home language Dutch } \\
\hline \multicolumn{12}{|l|}{10 No disability / disease } \\
\hline \multicolumn{12}{|l|}{$\begin{array}{l}11 \text { Grade in high school secondary education } \\
12 \text { Motivation before college }\end{array}$} \\
\hline 13 Years in higher education & $-0.03^{*}$ & & & & & & & & & & \\
\hline 14 Economics & $-0.04^{*}$ & $-0.04^{*}$ & & & & & & & & & \\
\hline 15 Education & $0.03^{*}$ & -0.01 & 0.00 & & & & & & & & \\
\hline 16 Agriculture & -0.01 & -0.01 & 0.00 & 0.00 & & & & & & & \\
\hline 17 Nature & $-0.02^{*}$ & 0.01 & 0.00 & 0.00 & 0.00 & & & & & & \\
\hline 18 Science & $-0.03^{*}$ & 0.01 & 0.00 & 0.00 & 0.00 & 0.00 & & & & & \\
\hline 19 Health & $0.07^{\star}$ & $0.06^{\star}$ & 0.00 & 0.00 & 0.00 & 0.00 & 0.00 & & & & \\
\hline 20 Law & $-0.03^{*}$ & $-0.02^{\star}$ & 0.00 & 0.00 & 0.00 & 0.00 & 0.00 & 0.00 & & & \\
\hline 21 Behavior & -0.01 & -0.01 & 0.00 & 0.00 & 0.00 & 0.00 & 0.00 & 0.00 & 0.00 & & \\
\hline 22 Language & $0.04^{*}$ & $-0.02^{\star}$ & 0.00 & 0.00 & 0.00 & 0.00 & 0.00 & 0.00 & 0.00 & 0.00 & \\
\hline Mean or Proportion & 4.29 & 2.61 & 0.14 & 0.02 & 0.06 & 0.11 & 0.15 & 0.19 & 0.05 & 0.16 & 0.11 \\
\hline SD & 0.78 & 1.16 & 0.34 & 0.13 & 0.24 & 0.32 & 0.36 & 0.40 & 0.22 & 0.37 & 0.32 \\
\hline Minimum & 1 & 1 & 0 & 0 & 0 & 0 & 0 & 0 & 0 & 0 & 0 \\
\hline Maximum & 5 & 4 & 1 & 1 & 1 & 1 & 1 & 1 & 1 & 1 & 1 \\
\hline
\end{tabular}


TABLE 2 Logistic Regression Estimates for Propensity Score Models

\begin{tabular}{llll}
\hline Variable & Coefficient (Exp B) & Standard error & p-Value \\
\hline SES def. 1 (1 = first generation) & $1.42^{\star \star}$ & 0.05 & $<.000$ \\
SES def. 2 (subjective social class) & 0.99 & 0.02 & .565 \\
Gender (1 = male) & $0.74^{\star \star}$ & 0.05 & $<.000$ \\
Age & 1.02 & 0.01 & .156 \\
Immigrant status (1 = immigrant status) & $0.54^{\star \star}$ & 0.11 & $<.000$ \\
Home language (1 = Dutch) & 0.96 & 0.09 & .668 \\
Disability or chronicle disease (1 = no) & 0.99 & 0.05 & .775 \\
Average grade high school & $0.19^{\star \star}$ & 0.62 & $<.000$ \\
Motivation before entrance to higher education & $1.24^{\star \star}$ & 0.03 & $<.000$ \\
Constant & $919.70^{\star *}$ & 2.20 & $<.000$ \\
\hline
\end{tabular}

${ }^{\star} p<.05 ;{ }^{*} p<.01^{\star \star}, N=21,452$

TABLE 3 Achieving Balance among Undermatched and Matched Students: Pre- and Post-test Matching T-tests Using 2-1 Nearest Neighbour Matching.

\begin{tabular}{|c|c|c|c|c|c|c|}
\hline & \multicolumn{3}{|c|}{ Unmatched sample } & \multicolumn{3}{|c|}{ Matched sample } \\
\hline & $\begin{array}{l}\text { Control } \\
\text { (Matched) }\end{array}$ & $\begin{array}{l}\text { Treatment } \\
\text { (Undermatched) }\end{array}$ & t-value & $\begin{array}{l}\text { Control } \\
\text { (Matched) }\end{array}$ & $\begin{array}{l}\text { Treatment } \\
\text { (Undermatched) }\end{array}$ & $\begin{array}{l}\text { t-val- } \\
\text { ue }\end{array}$ \\
\hline SES def. 2 ( 1 = first generation) & 0.29 & 0.41 & $12.08^{\star \star}$ & 0.39 & 0.41 & 1.62 \\
\hline SES def. 2 (subjective social class) & 6.80 & 6.65 & $-5.27^{\star \star}$ & 6.62 & 6.65 & 0.69 \\
\hline Gender $(1=$ male $)$ & 0.34 & 0.27 & $-7.48^{\star \star}$ & 0.28 & 0.27 & -1.01 \\
\hline Age & 21.58 & 21.59 & 0.10 & 21.58 & 21.59 & 0.19 \\
\hline Immigrant status (1 = immigrant status) & 0.06 & 0.04 & $-4.53^{\star \star}$ & 0.04 & 0.04 & -0.70 \\
\hline Home language (1 = Dutch) & 0.92 & 0.93 & 1.73 & 0.92 & 0.93 & 0.68 \\
\hline Disability or chronicle disease $(1=$ no) & 0.73 & 0.70 & $-2.56^{\star}$ & 0.71 & 0.70 & -0.51 \\
\hline Average grade high school & 7.26 & 6.84 & $-33.55^{\star \star}$ & 6.87 & 6.85 & -1.94 \\
\hline Motivation before entrance to higher education & 4.28 & $4 \cdot 37$ & $5 \cdot 76^{\star \star}$ & 4.36 & 4.37 & 0.54 \\
\hline N & 18626 & 2826 & & 5507 & 2826 & \\
\hline
\end{tabular}

${ }^{\star} p<.05,{ }^{\star \star} p<.01$

TABLE 4 Estimated coefficients (Beta) Predicting Student Satisfaction after Propensity Score Matching

\begin{tabular}{lllll}
\hline & \multicolumn{2}{l}{$\begin{array}{l}\text { Outcome: } \\
\text { Satisfaction with the social environment }\end{array}$} & \multicolumn{2}{l}{$\begin{array}{l}\text { Outcome: } \\
\text { Satisfaction with the academic environment }\end{array}$} \\
\cline { 2 - 4 } & $\beta$ & SE & $\beta$ & SE \\
\hline Undermatching (1 = undermatched) & $-0.12^{\star \star}$ & 0.02 & $-0.18^{\star \star}$ & 0.02 \\
Low-SES (1 = low) & -0.01 & 0.02 & -0.03 & 0.02 \\
Interaction: Low-SES $\times$ Undermatching & 0.01 & 0.03 & 0.03 & 0.03 \\
Constant & $3.98^{\star \star}$ & 0.02 & $3.78^{\star \star}$ & 0.02 \\
R-sq & 0.03 & & 0.03 & 8333 \\
$\mathrm{~N}$ & 8333 & & & \\
\hline
\end{tabular}

${ }^{\star} p<.05 ;{ }^{* \star} p<.01$. Standardized coefficients. Both models control for college major (8 dummies, results not shown). SES = socioeconomic status. 
Moreover, undermatching is related to less satisfaction with the academic and social environment. These relations are significant with modest effect sizes. These results indicate that regardless of SES, undermatching is related to lower satisfaction during college. These results show no evidence suggesting that undermatching may take along benefits in terms of more satisfaction for students with a low-SES background.

\section{Undermatching, SES, and student satisfaction during four years in higher education}

In order to test whether the relation between undermatching and satisfaction develops differently among low- and high-SES students during higher education, the interaction SES $\times$ undermatching $\times$ year (in addition to all relevant two-way interactions) was added to the models presented in Table 2. Results indicate a three-way interaction between SES, undermatching, and year 4 (with year 1 as reference category) (see Table 5) with regard to satisfaction with the academic environment, but not regarding satisfaction with the social environment. In order to interpret this significant interaction, exploratory analyses for each separate year in higher education (year 1, 2, 3, and 4) were conducted, assessing simple mean comparisons. These analyses, shown in Figure 1, reveal that this significant interaction indicates that among matched students, low- and high-SES students are equally satisfied by the fourth year of college. On the contrary, among undermatched students, the low-SES students become less satisfied than their high-SES fellow students by the fourth year of college.

Importantly, these simple mean comparisons reveal that undermatched students (both low- and high SES students) are less satisfied with the social and academic environment than matched students in most academic years. In addition, these differences become more pronounced toward the fourth year in college, especially for low-SES students. In the fourth year in college, undermatched low-SES students are on average 0.43 point less satisfied with the academic environment, and 0.25 point less satisfied with the social environment than matched students on a 5-point scale (results shown in Figures 1 and 2).

Results from all models show the same pattern when applying the alternative definition of SES (subjective SES); therefore, these results are not shown.

\section{Sensitivity analyses}

The results of analyses after propensity score matching are presented, a technique that addresses issues concerning endogeneity. Although this procedure addresses possible confounding effects from covariates, it may at the same time harm the generalizability of the findings. After all, the matching procedure excluded participants in the control group (matched students) who have no counterpart in the treatment group because of their estimated propensity score. Therefore, a sensitivity analysis was run, by applying a conventional linear regression model predicting student satisfaction, with all covariates as controls, including the whole sample $(N=21,452)$. Results show the same pattern as the 
results found after applying the PSM procedure, indicating that the results are not biased by the selection based on the PSM. Therefore, results showed no evidence to suggest that the findings do not generalize to the whole student sample (see Appendix A, Table A1).

TABLE 5 Estimated coefficients (Beta) Predicting Student Satisfaction after Propensity Score Matching: Interaction between SES, Undermatch, and Year

\begin{tabular}{|c|c|c|c|c|}
\hline & \multicolumn{2}{|c|}{$\begin{array}{l}\text { Satisfaction with the social } \\
\text { environment }\end{array}$} & \multicolumn{2}{|c|}{$\begin{array}{l}\text { Satisfaction with the aca- } \\
\text { demic environment }\end{array}$} \\
\hline & $\beta$ & SE & $\beta$ & SE \\
\hline \multicolumn{5}{|l|}{ Predictors } \\
\hline Undermatching (1 = undermatched) & $-0.06^{*}$ & 0.03 & $-0.12^{\star \star}$ & 0.04 \\
\hline Low-SES, objective (1 = low-SES) & -0.01 & 0.03 & -0.04 & 0.04 \\
\hline \multicolumn{5}{|l|}{ Year (year 1 = ref) } \\
\hline Year 2 & -0.01 & 0.02 & $-0.05^{*}$ & 0.02 \\
\hline Year 3 & -0.03 & 0.03 & $-0.08^{\star *}$ & 0.02 \\
\hline Year 4 & -0.01 & 0.02 & $-0.05^{*}$ & 0.02 \\
\hline \multicolumn{5}{|l|}{ Three-way interactions } \\
\hline Interaction SES $\times$ Undermatching $\times$ Year 2 & 0.00 & 0.03 & 0.00 & 0.02 \\
\hline Interaction SES $\times$ Undermatching $\times$ Year 3 & 0.04 & 0.02 & 0.01 & 0.02 \\
\hline Interaction SES $\times$ Undermatching $\times$ Year 4 & -0.03 & 0.02 & $-0.05^{*}$ & 0.02 \\
\hline Constant & $4.00^{\star \star}$ & 0.03 & $3.85^{\star \star}$ & 0.03 \\
\hline R-sq & 0.04 & & 0.04 & \\
\hline $\mathrm{N}$ & 8333 & & 8333 & \\
\hline
\end{tabular}

Note. ${ }^{*} p<.05 ;{ }^{* \star} p<.01$. Standardized coefficients. Both models also included college major as covariates and all two-way interactions regarding undermatching, SES, and Year (results not shown).

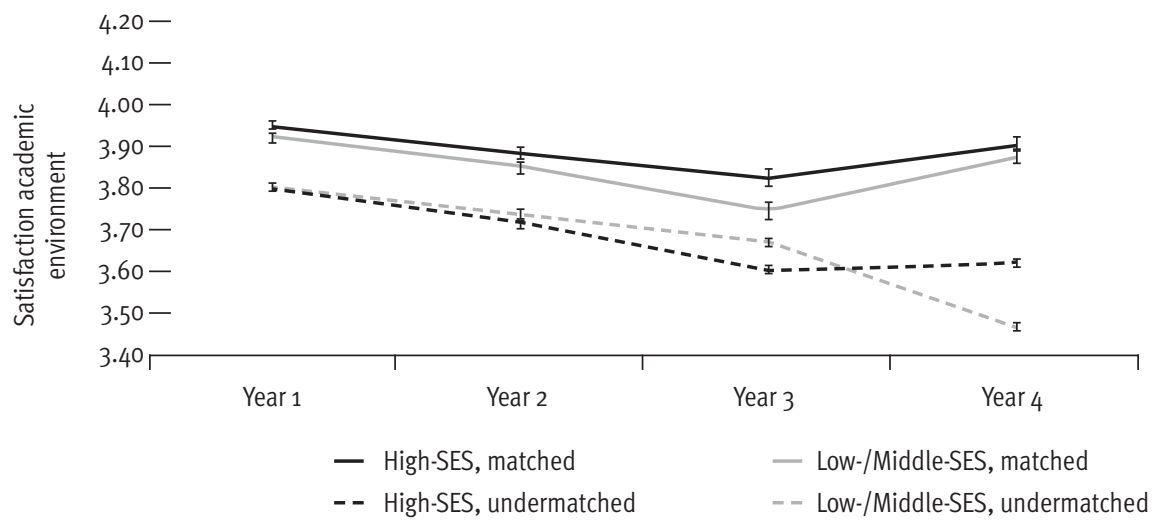

FIGURE 1 Satisfaction with the academic environment (scale 1-5) during the years in higher education among matched- and undermatched low-and high-SES students (objective SES, cross-sectional data) after propensity score matching, $\mathrm{N}=8,333$. 


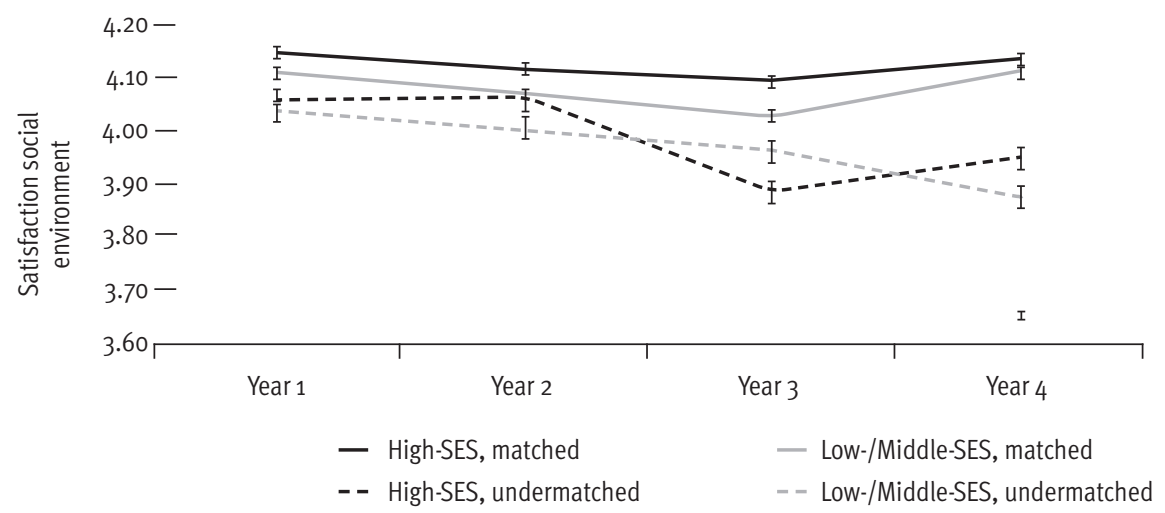

FIGURE 2 Satisfaction with the social environment (scale 1-5) during the years in higher education among matched- and undermatched low-and high-SES students (objective SES, cross-sectional data) after propensity score matching, $\mathrm{N}=8,333$.

\subsection{Discussion}

Undermatching, when students attend post-secondary institutions which are less selective than their academic credentials would permit, is generally considered as an undesirable outcome because of the long-term consequences for students' job opportunities and wages (Ovink et al., 2018), especially for low-SES students, who are more likely to undermatch (Bastedo \& Jaquette, 2011). However, there is a gap in the literature regarding the relation between undermatching and students' subjective experiences during college, and its relation with SES. Studying adolescents' subjective experiences during their years in college is important because of its consequences for college completion (Bowen et al., 2009), and because of the accumulating effects that both positive and negative experiences can have in their development toward adulthood (Yoshioka \& Noguchi, 2009). The literature suggests that when students are undermatched, the academic demands from their institutions are misaligned with their potential (e.g., Hoxby \& Turner, 2013), which may lead to less satisfaction with the academic environment. However, experiences of social mismatch and feeling 'out of place' when low-SES students enter the most selective institutions are well documented (Jury et al., 2017). The cultural codes in less selective institutions may match better with low-SES students than the cultural codes in highly selective institutions (Deutschlander, 2017). Consequently, there may be a positive relation between undermatching and satisfaction with the social environment, but only among low-SES students.

In the present study, the relation between undermatching and satisfaction, and the moderating role of SES, was investigated with a large, representative Dutch dataset that includes information about student self-reported satisfaction and student characteristics 
such as age, motivation, and grades during high school ( $\mathrm{N}=21,452$ respondents). Up till now, undermatching has been studied mainly in the U.S., where undermatching has to be estimated from institutions' selectivity levels, and students' eligibility to these institutions. Because there are many different ways to define these constructs, concerns have raised regarding comparability and accuracy of these estimations (Rodriguez, 2015). In the Netherlands, both institutional selectivity and students' qualifications are much easier to determine, leading to highly accurate and undebatable estimations of undermatching,

The study examined satisfaction among low-SES students all of whom are eligible for the most selective institutions, but who are either in the most selective institutions (match), or in less selective institutions (undermatch). To test whether any effects are specific to lowSES students, their satisfaction was compared with the satisfaction of high-SES students in both selective and non-selective institutions. In addition, it was examined whether these relations (both for low-and high-SES students) change throughout the four years in higher education. Because pre-existing differences may confound the relation between undermatching and satisfaction, propensity score matching (PSM) was applied to test the consequence of undermatching, excluding as much as possible the confounding influences of covariates.

The present study findings show that undermatching is related to less satisfaction with the academic and social environment, and that this relation becomes stronger toward the fourth year in higher education. The study did not provide any evidence showing that undermatching is related to more satisfaction among low-SES students. These results do not only suggest that there are no benefits for low-SES students related to undermatching, undermatching even seems to have costs in terms of less satisfaction with the social and academic environment during college, especially toward the later years in higher education.

\section{Undermatching and academic and social satisfaction}

The finding that undermatched student are less satisfied with the academic environment (i.e., the content and rigorousness of the educational program) is in line with literature that suggests that de demands from less selective institutions are misaligned with the capacities of undermatched students (Belasco \& Trivette, 2015). In less selective institutions, undermatched students are probably not maximizing their full potential (Hoxby \& Turner, 2013). Previous research has shown that students have higher chances of graduating if the quality level of their institution matches their observed skill levels (Light \& Strayer, 2000). The lower satisfaction after undermatching shown in the present study may be an important factor in the relation between undermatching and degree attainment.

The finding that low-SES students' satisfaction with the social environment does not benefit from undermatching, indicates that there may also be a social mismatch when lowSES students attend less selective institutions in higher education. In the less selective institutions, the proportion low-SES students is higher than in the most selective institutions 
(Bastedo \& Jaquette, 2011) (i.e., 40\% low-SES students in less selective tracks versus $25 \%$ low-SES students in the most selective tracks in the Netherlands; Dutch Inspectorate of Education, 2018). As a consequence, cultural codes in less selective institutions may match better with low-SES students' backgrounds (Walpole, 2003). However, the results suggest that the larger proportion of other low-SES students seem not to elevate their satisfaction regarding experiences with the social environment. This finding may suggest a social mismatch in all higher education institutions, regardless of the level of selectivity. In addition, the finding that students who are undermatched, experience less satisfaction with the social environment, suggests that undermatching does not take along benefits in terms of satisfaction with social aspects of college, both for low- and high-SES students.

The finding that the negative relationship between undermatching and satisfaction (social and academic) seems to manifest in the later years in college, suggests that undermatching has especially consequences after students have integrated in their new college. When this negative relationship would have been strongest in their first year, this might have been related to a process of adjustment related to separation from the old situation and transition into the new college. However, the enhanced negative relationship after the phase of transition, seems to reflect how students feel about their situation once they adjusted. Although speculative, this finding may also predict a negative relationship between undermatching and satisfaction on the job market, after college.

The present study findings show that low-SES students seem not to benefit from undermatching in terms of satisfaction. Endogeneity could lead to an overestimation of the relation between undermatching and satisfaction. For example, students who are not motivated to enter higher education may be more likely to undermatch and to become dissatisfied. In addition, students who are less cognitively talented may be more likely to undermatch and become less satisfied during higher education. Nevertheless, because students with the same eligibility for the most selective institution were selected, and because PSM was applied to exclude the confounding effects of covariates, such as motivation for college and grades during high school, endogeneity is unlikely to explain the current findings.

These findings add to the body of research on the consequences of undermatching. Although there are several differences between the U.S. context and the European context in higher education, the basic principles underlying undermatching (i.e., students attend less selective institutions than their academic credentials would permit) are the same in many regards. First, both in the U.S. and in Europe, the eligibility for the most selective institutions depends on academic performance during middle adolescence. Second, an important similarity is that attending less selective institutions is on average related to less prestigious jobs and lower wages on the long term. Third, both in the U.S. and Europe, low-SES students tend to undermatch more than high-SES students. Fourth, both in the U.S. and in Europe, students' years in college are usually spent during late adolescence, covering the same developmental stage toward early adulthood. Therefore, it is plausible that the results of the present study are generalizable to the U.S. context. 


\section{Implications}

The present study extends the knowledge about the effects of undermatching by showing that also in the short term, during college, undermatching affects students' wellbeing. These results are of important because of low-SES students' higher likelihood to undermatch (Belasco \& Trivette, 2015). The less positive college experiences related to undermatching may reinforce educational disadvantage for students from low-SES backgrounds. First, the lower satisfaction may have negative consequences for their college completion (Ovink et al., 2018). Second, these enduring experiences of lower satisfaction during college increase the likelihood of encountering stressful experiences related to a low socioeconomic background (Wickrama, O’Neal, Lee, \& Wickrama, 2015). This accumulation of stressful experiences during adolescence can have detrimental consequences for health and well-being in adulthood (Wickrama, et al., 2016), especially for social mobile adolescents (Miller, Yu, Chen, \& Brody, 2015; Wickrama et al., 2016). In addition, the finding that the negative relationship between undermatching and satisfaction enhances toward the later years in college, suggests that this relationship manifests after students' integration in college. Although speculative, this finding may also suggest a negative relation between undermatching and job-satisfaction after graduation.

Clearly, these findings have also implications for the formulation of policies and programs for promoting social mobility. Undermatching arises during the transition from high school to the most selective institutions and is related to a wide range of barriers (Page \& ScottClayton, 2016). Traditionally, the knowledge deficit approach states that students' choice to undermatch is a result of a lack of information about application processes and college costs. Research on college choice processes indeed shows that low-SES students' tendency to undermatch is highly related to having less access to information about institutions compared to high-SES students; low-SES students are less likely to undermatch when they receive high-quality information about their possibilities (Hoxby \& Avery, 2013). However, even with access to 'perfect information', undermatching still occurs among lowSES students (Black, Cortes, \& Lincove, 2015). The preference approach to undermatch explains this tendency by differences between low- and high-SES students in factors that students take into account during their college decision-making, like geographic factors, college fit, and opinions of relatives and peers (Black et al., 2015; Tiboris, 2014). From this perspective, it has been argued that undermatching can be the result of a well-informed, autonomous decision (Tiboris, 2014). In sum, both the knowledge deficit approach and the preference approach suggest that it is important to offer low-SES students highquality information during the transition to higher education. Policy on social equality has encouraged high schools to improve information during the college choice process. The present study indicates that low-SES students should also be informed about their higher risk on lower satisfaction during the later years in higher education when they are undermatched. 


\section{Limitations}

Despite the importance of these findings, the present study has several limitations. One limitation is that the data are cross-sectional, and therefore, it cannot with certainty be concluded whether the differences between the years are actually reflecting student development during these years. For example, students who are very dissatisfied may leave higher education, resulting in a biased estimation of satisfaction from year 1 to year 4. However, student drop-out peaks after the first year in higher education: 33\% switches or drops out after the first year. Yet, among students who continue after their first year, $86 \%$ obtains their diploma (Dutch Inspectorate of Education, 2018). Therefore, it is plausible that the data capture student development over years, especially in the later years of higher education when drop-out rates are low. However, longitudinal data are necessary to better map this development.

Moreover, although applying PSM in order to exclude confounding effects of covariates is a highly recommended method to approach the relation between undermatching and satisfaction as close as possible, there might be unobserved confounders. For example, personality traits may also partly determine whether students undermatch or match, and these were not measured. Therefore, despite the use of PSM methodology, it is important to remain cautious with causal interpretations.

Next, certain aspects of students' experiences in college that may influence their satisfaction, such as the possibility to engage in collaborative learning activities or in extracurricular events, were not measured. Some recent studies with small samples of first students suggest positive effects of undermatching on college experiences among first-year ethnic minorities (Fosnacht, 2014, 2015; Lowry, 2017), because undermatched students engage more in active and collaborative learning activities in less selective institutions. Especially black students were found to be less affected or even to benefit from undermatching. Because of limitations in the dataset, it was not possible to study the role of these college experiences, nor ethnicity, conclusively. Therefore, it is possible that undermatching can contribute positively to students' subjective experiences when institutions offer certain social activities.

Furthermore, the reason for students to undermatch may vary across students and affect satisfaction. For example, low-SES students are likely to undermatch for the reason that they can stay closer to their family and friends (Belasco \& Trivette, 2015). The motives for students to undermatch may moderate the negative relation between undermatching and satisfaction. In this study, the role of the reason to undermatch could not be tested because this was not measured in the dataset. Initial differences between matched and undermatched students, such as motives to undermatch or self-efficacy, although not of explicit interest in this study, are of potential interest in future research. 


\section{Conclusion}

Students who attend less selective institutions in higher education than they are eligible for (undermatching) tend to achieve less job opportunities and lower wages in the long term than students who do not undermatch (Ovink et al., 2018). Therefore, in the literature, undermatching is mainly regarded as an undesirable outcome (e.g., Hoxby \& Turner, 2013), especially among low-SES students, who are more likely to undermatch (Belasco \& Trivette, 2015). Because of the importance of subjective experiences during adolescents for their development toward adulthood (Yoshioka \& Noguchi, 2009), and the gap in the literature regarding undermatching, SES, and student satisfaction, the current study tested with a large-scale cohort study 21,452 respondents ( $67 \%$ female) among adolescents how undermatching is related to students' satisfaction in college by using propensity score matching (PSM, Thoemmes \& Kim, 2011). Results showed a negative relation between undermatch and satisfaction with the social and the academic environment that increases toward the fourth year in college. This relation appeared to be negative for both low- and high-SES students. For example, undermatched students, regardless of their SES, are at the end of the first academic year less satisfied with the academic environment than matched students. In addition, their satisfaction lowers during the first year whereas the satisfaction among matched students remains stable. These findings have important implications for the understanding about undermatching in relation to students' development, and for policy interventions. The higher likelihood to undermatch among students from low-SES backgrounds may reinforce their educational disadvantage, because the lower satisfaction tied to undermatching may have negative consequences for their college completion (Bowen et al., 2009), and because enduring stressful experiences during adolescence can have harmful consequences for their health and well-being in adulthood (Miller et al., 2015; Wickrama et al., 2016). The finding that the negative relationship between undermatching and satisfaction seems to increase after integration into college, suggests that the mismatch may also continue after graduation, on the labor market. Research on reasons to undermatch has shown that high-quality information is important in helping students to make appropriate decisions during their transition into higher education (Black et al., 2015; Hoxby \& Avery, 2013). Therefore, it is important that students, especially when they have a low-SES background, are informed about the negative relation between undermatching and satisfaction toward the later years in higher education. 


\subsection{References}

Aljohani, O. (2016). A comprehensive review of the major studies and theoretical models of student retention in higher education. Higher Education Studies, 6(2), 1-18.

Austin, P. C. (2010). Statistical criteria for selecting the optimal number of untreated subjects matched to each treated subject when using many-to-one matching on the propensity score. American Journal of Epidemiology, 172(9), 1092-1097.

American Psychological Association, Task Force on Socioeconomic Status. (2007). Report of the APA Task Force on Socioeconomic Status. Washington, DC.

Bastedo, M. N., \& Jaquette, O. (2011). Running in place: Low-income students and the dynamics of higher education stratification. Educational Evaluation and Policy Analysis, 33(3), 318-339.

Belasco, A. S., \& Trivette, M. J. (2015). Aiming low: Estimating the scope and predictors of postsecondary undermatch. The Journal of Higher Education, 86(2), 233-263.

Black, S. E., Cortes, K. E., \& Lincove, J. A. (2015). Apply yourself: Racial and ethnic differences in college application (No. w21368). National Bureau of Economic Research.

Bowen, W. G., Chingos, M. M., \& McPherson, M. S. (2009). Crossing the finish line: Completing college at America's public universities (Vol. 52). Princeton University Press.

Bradshaw, M., Kent, B. V., Henderson, W. M., \& Setar, A. C. (2017). Subjective social status, life course SES, and BMI in young adulthood. Health Psychology, 36(7), 682-694.

Davidson, C., \& Wilson, K. (2013). Reassessing Tinto's concepts of social and academic integration in student retention. Journal of College Student Retention: Research, Theory \& Practice, 15(3), 329-346.

Davis-Kean, P. E. (2005). The influence of parent education and family income on child achievement: the indirect role of parental expectations and the home environment. Journal of Family Psychology, 19(2), 294-304.

Deutschlander, D. (2017). Academic undermatch: How general and specific cultural capital structure inequality. Sociological Forum, 32(1), 162-185.

Dutch inspectorate of education (2018). The State of Education in the Netherlands 2016/2017. Utrecht: Dutch inspectorate of education.

Ellis, B. J., Bianchi, J., Griskevicius, V., \& Frankenhuis, W. E. (2017). Beyond risk and protective factors: An adaptation-based approach to resilience. Perspectives on Psychological Science, 12(4), 561-587.

Fosnacht, K. (2014). Selectivity and the college experience: How undermatching shapes the college experience among high-achieving students. Presented at the annual meeting of the American Educational Research Association, Philadelphia, PA.

Fosnacht, K. (2015). Undermatching and the first-year experience: Examining effect heterogeneity. Denver, Colorado.

Frankenhuis, W. E., \& Nettle, D. (2018). Open science is liberating and can foster creativity. Perspectives on Psychological Science, 13(4), 439-447.

Howell, J. S., \& Pender, M. (2016). The costs and benefits of enrolling in an academically matched college. Economics of Education Review, 51, 152-168.

Hoxby, C., \& Avery, C. (2013). The missing "one-offs": The hidden supply of high-achieving, lowincome students. Brookings papers on economic activity, 2013(1), 1-65. 
Hoxby, C. M., \& Turner, S. (2013). Expanding college opportunities for high achieving, low income students (SIEPR 12-014). Stanford, CA: Stanford Institute for Economic Policy Research.

lacus, S. M., King, G., \& Porro, G. (2011). Multivariate matching methods that are monotonic imbalance bounding. Journal of the American Statistical Association, 106(493), 345-361.

Jæger, M. M. (2009). Equal access but unequal outcomes: Cultural capital and educational choice in a meritocratic society. Social Forces, 87(4), 1943-1971.

Jury, M., Smeding, A., Stephens, N. M., Nelson, J. E., Aelenei, C., \& Darnon, C. (2017). The experience of low-SES students in higher education: Psychological barriers to success and interventions to reduce social-class inequality. Journal of Social Issues, 73(1), 23-41.

Jussim, L., Eccles, J., \& Madon, S. (1996). Social perception, social stereotypes, and teacher expectations: Accuracy and the quest for the powerful self-fulfilling prophecy. Advances in Experimental Social Psychology, 28, 281-388.

Kuncel, N. R., Credé, M., \& Thomas, L. L. (2005). The validity of self-reported grade point averages, class ranks, and test scores: A meta-analysis and review of the literature. Review of Educational Research, 75(1), 63-82.

Lee, V. E., \& Burkam, D. T. (2002). Inequality at the starting gate: Social background differences in achievement as children begin school. Economic Policy Institute, Washington DC.

Light, A., \& Strayer, W. (2000). Determinants of college completion: School quality or student ability? The Journal of Human Resources, 35(2), 299-332.

Lowry, K. M. (2017). Community college choice and the role of undermatching in the lives of African Americans. Community College Journal of Research and Practice, 41(1), 18-26.

Mayhew, M. J., Rockenbach, A. B., Bowman, N. A., Seifert, T. A., \& Wolniak, G. C. (2016). How college affects students: 21st Century evidence that higher education works. San Francisco: Jossey-Bass.

Miller, G. E., Yu, T., Chen, E., \& Brody, G. H. (2015). Self-control forecasts better psychosocial outcomes but faster epigenetic aging in low-SES youth. Proceedings of the National Academy of Sciences, 112(33), 10325-10330.

Mullainathan, S., \& Sharif, E. (2013). Scarcity: Why having too little means so much. London: Allen Lane.

Ovink, S., Kalogrides, D., Nanney, M., \& Delaney, P. (2018). College match and undermatch: Assessing student preferences, college proximity, and inequality in post-college outcomes. Research in Higher Education, 59(5), 553-590.

Page, L. C., \& Scott-Clayton, J. (2016). Improving college access in the United States: Barriers and policy responses. Economics of Education Review, 51, 4-22.

Thoemmes, F., \& Kim, E. S. (2011). A systematic review of propensity score methods in the social sciences. Multivariate Behavioral Research, 46, 90-118

Roderick, M., Nagaoka, J., Allensworth, E., Coca, V., Correa, M., \& Stoker, G. (2006). From high school to the future: A first look at Chicago public school graduates' college enrollment, college preparation, and graduation from four-year colleges. Chicago: Consortium on Chicago School Research.

Rodriguez, A. (2015). Tradeoffs and limitations: Understanding the estimation of college undermatch. Research in Higher Education, 56(6), 566-594. 
Rosenbaum, P. R., \& Rubin, D. B. (1983). The central role of the propensity score in observational studies for causal effects. Biometrika, 70(1), 41-55.

Singh-Manoux, A., Adler, N. E., \& Marmot, M. G. (2003). Subjective social status: Its determinants and its association with measures of ill-health in the Whitehall II study. Social Science \& Medicine, 56(6), 1321-1333.

Sirin, S. R. (2005). Socioeconomic status and academic achievement: A meta-analytic review of research. Review of Educational Research, 75(3), 417-453.

Smith, J., Pender, M., \& Howell, J. (2013). The full extent of student-college academic undermatch. Economics of Education Review, 32, 247-261.

Stephens, N. M., Townsend, S. S., \& Dittmann, A. G. (2018). Social-class disparities in higher education and professional workplaces: The role of cultural mismatch. Current Directions in Psychological Science, 28(1), 67-73.

Tiboris, M. (2014). What's wrong with undermatching? Journal of Philosophy of Education, 48(4), 646-664.

Tinto, V. (1975). Dropout from higher education: A theoretical synthesis of recent research. Review of Educational Research, 45(1), 89-125.

Thoemmes, F. J., \& Kim, E. S. (2011). A systematic review of propensity score methods in the social sciences. Multivariate Behavioral Research, 46(1), 90-118.

Van den Broek, A., de Korte, K, Cuppen, J., Wartenbergh, F., Bendig-Jacobs, J., Mulder, J., Hellegers, A. (2018). Monitor Beleidsmaatregelen 2017-2018: Studiekeuze, studiegedrag \& leengedrag in relatie tot beleidsmaatregelen in het hoger onderwijs. Nijmegen: ResearchNed.

Wickrama, K. A. S., O’Neal, C. W., Lee, T. K., \& Wickrama, T. (2015). Early socioeconomic adversity, youth positive development, and young adults' cardio-metabolic disease risk. Health Psychology, 34(9), 905-914.

Wickrama, K. A., O'Neal, C. W., \& Lee, T. K. (2016). The health impact of upward mobility: does socioeconomic attainment make youth more vulnerable to stressful circumstances? Journal of Youth and Adolescence, 45(2), 271-285.

Walpole, M. (2003). Socioeconomic status and college: How SES affects college experiences and outcomes. The Review of Higher Education, 27, 45-73.

Yoshioka, M. R., \& Noguchi, E. (2009). The developmental life course perspective: A conceptual and organizing framework for human behavior and the social environment. Journal of Human Behavior in the Social Environment, 19(7), 873-884. 


\subsection{Appendix A}

TABLE A1 Sensitivity Analyses: Estimated coefficients (Beta) Predicting Student Satisfaction without Propensity Score Matching

\begin{tabular}{|c|c|c|c|c|}
\hline & \multicolumn{2}{|c|}{$\begin{array}{l}\text { Outcome: } \\
\text { Satisfaction with the social } \\
\text { environment }\end{array}$} & \multicolumn{2}{|c|}{$\begin{array}{l}\text { Outcome: } \\
\text { Satisfaction with the } \\
\text { academic environment }\end{array}$} \\
\hline & $\beta$ & SE & $\beta$ & SE \\
\hline \multicolumn{5}{|l|}{ Predictors } \\
\hline Undermatching (1 = undermatched) & $-0.06^{\star \star}$ & 0.01 & $-0.10^{\star \star}$ & 0.01 \\
\hline Low-SES (1 = low) & $-0.02^{*}$ & 0.01 & $-0.02^{*}$ & 0.01 \\
\hline Interaction: Low-SES × Undermatching & 0.01 & 0.01 & 0.00 & 0.01 \\
\hline \multicolumn{5}{|l|}{ Covariates } \\
\hline Gender $(1=$ male $)$ & $0.02^{\star}$ & 0.01 & 0.01 & 0.01 \\
\hline Age & $-0.04^{\star \star}$ & 0.01 & $-0.03^{\star \star}$ & 0.01 \\
\hline Immigrant status (1 = immigrant status) & $-0.05^{\star \star}$ & 0.01 & -0.01 & 0.01 \\
\hline Home language (1 = Dutch) & $0.04^{\star \star}$ & 0.01 & $0.02^{*}$ & 0.01 \\
\hline Disability or chronicle disease $(1=$ no) & $0.03^{\star \star}$ & 0.01 & $0.02^{*}$ & 0.01 \\
\hline Average grade high school & $0.05^{\star *}$ & 0.01 & $0.02^{*}$ & 0.01 \\
\hline Motivation before higher education & $0.16^{\star \star}$ & 0.01 & $0.17^{\star \star}$ & 0.01 \\
\hline Constant & $3 \cdot 54^{\star \star}$ & 0.37 & $3.42^{\star \star}$ & 0.39 \\
\hline R-sq & 0.07 & & 0.06 & \\
\hline N & 21,452 & & 21,452 & \\
\hline
\end{tabular}

${ }^{\star} p<.05 ;{ }^{* \star} p<.01$. Standardized coefficients. Both models control for college major (8 dummies, results not shown). SES = socioeconomic status. 



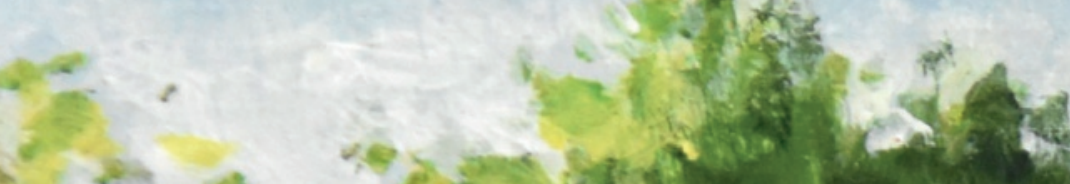

tes is un wat

$2 x-4 x^{2}$

\section{$2=$ \\ SW \\ $x_{2} \cos ^{2}$ \\ $8 x$ \\ $\approx$}

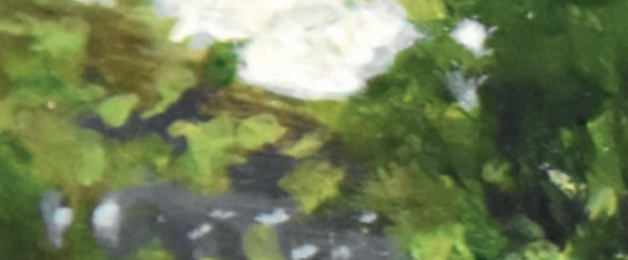

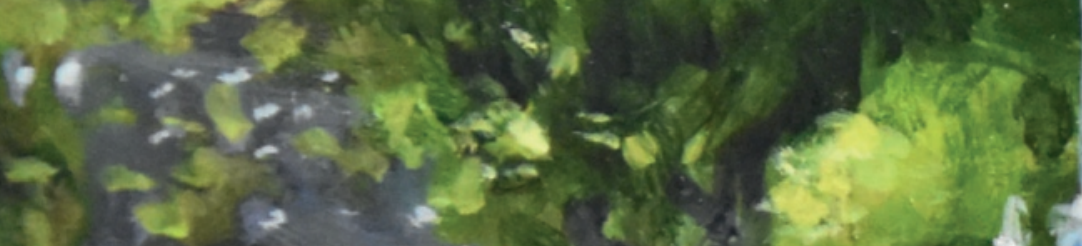

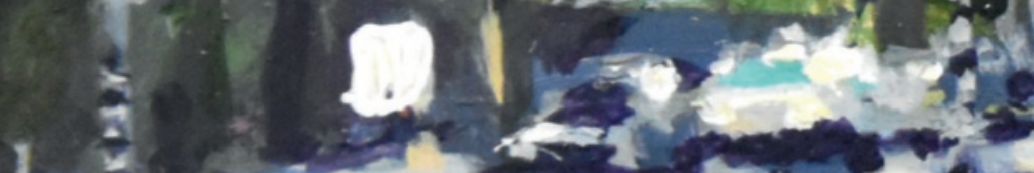

$1 \frac{2}{3}=2$

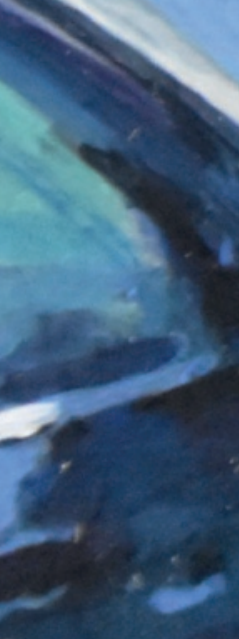




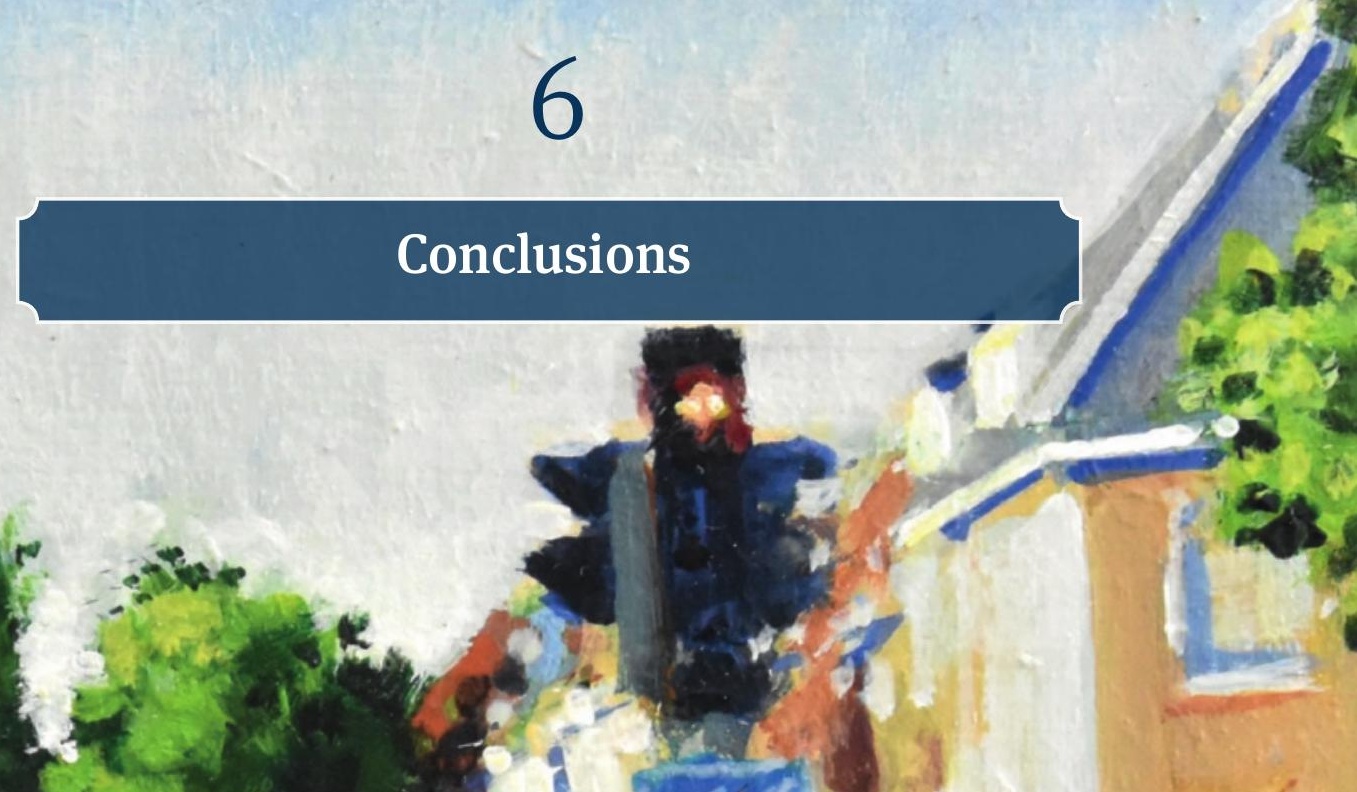

\section{Conclusions}

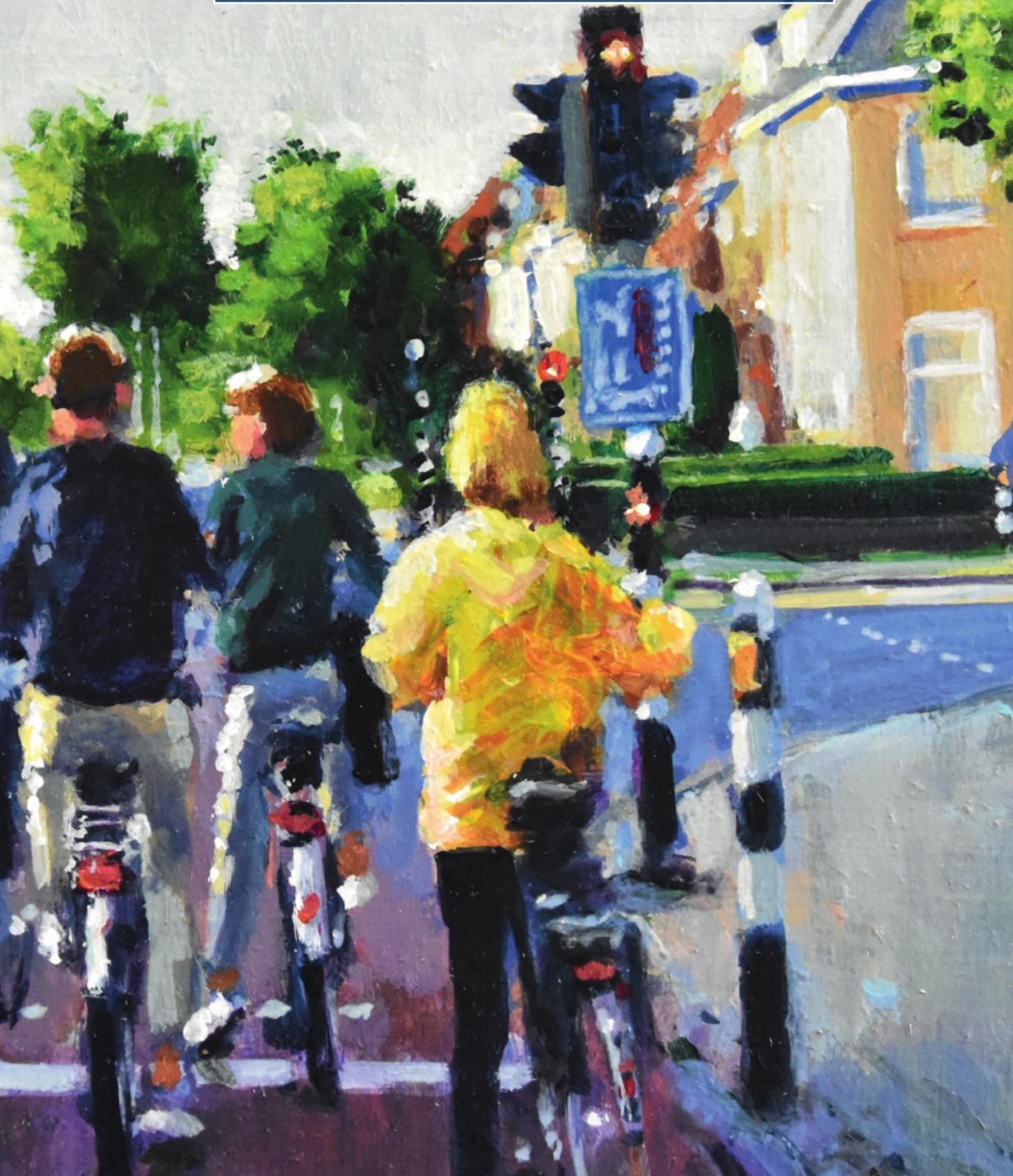




\subsection{Summary of the main findings}

The aim of this thesis is to investigate the existence and consequences of hidden barriers that students from low socioeconomic status (SES) encounter in educational settings. It is well documented that students' SES-background is a key predictor for academic performance and educational attainment (Sirin, 2005). Low socioeconomic circumstances have detrimental effects on cognitive development, parental resources, and educational opportunities. These effects are known to contribute to the relation between SES and educational attainment (Mani, Mullainathan, Shafir, \& Zhao, 2013; Vohs, 2013). However, less is known about the possibility that the relation between SES-background and educational attainment is also reinforced by a mismatch between low-SES students' skills and strengths, and features of educational environments (e.g., Ellis, Bianchi, Griskevicius, \& Frankenhuis, 2017; Rogoff et al., 2017; Valencia, 2010). Demands in school environments may be unintendedly tailored towards students from high-SES backgrounds, disadvantaging low-SES students (e.g., Rogoff et al., 2017; Sternberg, 2005; Valencia, 2010). This thesis adds to the current literature by investigating empirically the existence and consequences of mismatches related to students' socioeconomic background across three qualitatively different domains. Specifically, possible mismatches are examined with regard to the content of test materials offered to students, to personality traits, and to the study environment more broadly. First, I focus on features of math tests that may unintendedly bias low-SES students' performance. Second, I investigate whether the well-known positive relationship between certain personality traits (e.g., Openness) and educational outcomes differs by students' SES-background. Finally, I study the consequences from undermatching (i.e., when students attend less selective colleges than their credentials would permit) on students' subjective experiences, and focus on the possibility that these consequences differ by students' SES.

Chapter 2 shows that regardless of their level of intelligence, certain context (i.e., money, social interaction) applied in math-test items to explain mathematical problems, may impede low-SES students' performance. Neutral context, mathematical notation, and context related to food, were not related to impaired performance among low-SES students. These results suggest new insights in features of standardized tests that disadvantage low-SES students, and might have significant implications for fair testing.

Chapter 3 shows that the personality trait Openness in late childhood predicts educational attainment in middle adolescence, and that this relation is stronger for low-SES students than for high-SES students. These results suggest that the personality trait Openness may compensate for background disadvantage. Because we find this pattern with regard to performance related to teachers' subjective evaluations, but not with regard to students' objective performance, these findings may point to the possibility that this compensating role might work through teachers' evaluations. For low-SES students, who are more likely to meet low teacher expectations, displaying Openness may be a way to influence teachers' expectations. 
Chapter 4 shows that in their first year in higher education, students from low-SES environments who enroll in the most selective institutions in higher education experience a match with the academic environment, but at the same time experience a mismatch with the social environment. Undermatched low-SES students (i.e., students who are enrolled in less selective institutions than they are eligible for) are not experiencing this mismatch with the social environment during their first year. We conclude that undermatching may contribute to students' integration into college during their first year, but only among lowSES students.

Chapter 5 shows that students in higher education that are undermatched are in general less satisfied with their social and academic environment. The negative relation between undermatching and satisfaction increases toward the last year in college, especially for low-SES students. This lowered satisfaction in the final stage in higher education implies that the negative consequences of undermatching become more pronounced after students have become more integrated into their colleges. In sum, whereas undermatching may positively contribute to the social integration of low-SES students in higher education during their first year in college (chapter 4), undermatching seems to lead to less satisfaction with social and academic aspects in college toward the final stage in higher education (chapter 5).

The research reported in this dissertation made use of preregistration of research questions, data source, definitions, and statistical plans, in line with recent recommendations (Wagenmakers, Wetzels, Borsboom, van der Maas, \& Kievit, 2012). The current view in psychology on preregistration is that it reduces the risk of false positives, and that preregistered research is more likely to produce findings that replicate, compared to traditional research (Nelson, Simmons, \& Simonsohn, 2017). In Chapter 2, 4, and 5 , preregistrations were published on Open Science Framework (https://osf.io). Chapter 3 is a follow-up on a commissioned research by order of the Dutch Ministry of Education, and research questions and the data source were discussed and established in a research proposal before conducting the analyses. In all studies, the initial plan changed during the research process, for example because of new insights based on relevant literature or reviewers' comments, unforeseen flaws in the data, or new research questions that were evoked by the results. Therefore, in all studies, we added additional information about these deviations to the preregistration and/or to the manuscript, or decided to conduct additional exploratory analyses. Because of the preregistration, such changes are now systematically recorded during each research project. Furthermore, through preregistration, the distinction between confirmatory and exploratory analyses is clear for each chapter. Being able to make this distinction is important because it improves the accuracy of inferences, and therefore the likelihood that findings will replicate (Wagenmakers et al, 2012).

In sum, this dissertation shows new evidence for the existence and consequences of hidden barriers for low-SES students in educational contexts. It reveals several features of educational environments that may have differential consequences for students from 
low-and high-SES backgrounds including test materials used, interaction with personality traits, and the encountered social and academic environment. Together, the findings imply that focusing on mismatches that may occur when low-SES students attend schools or institutions for higher education, may lead to more insight in the negative relation between low socioeconomic background and academic performance. These insights may have implications for policy aimed at reducing the academic performance gap between lowand high-SES students. Moreover, with regard to research practices, the experiences with preregistration lead to policy recommendations as well. The main policy implications are discussed in the next section.

\subsection{Implications, Knowledge Valorisation, and Recommendations}

We found indications for hidden barriers in math-tests for low-SES students. Applying context such as money or social interaction seems to work against low-SES students' performance. When future research would replicate these findings and show that applying this context impedes low-SES students' performance, these barriers should be addressed with policy interventions. It may be recommendable for test developers to pay attention to the risk of biasing effects from context in math tests. Omitting context such as money and social interaction from standardized math-tests may lead to a reduction of the SES-gap in math performance.

In addition, we found indications that when low-SES students display high levels of Openness, this may positively influence teachers' evaluations about their abilities, compensating for their background disadvantage. Policy implications of this study are twofold. First, when this relationship turns out to be causal, strengthening beneficial personality traits during childhood, in particular Openness, may help to elevate teachers' expectations toward low-SES students, perhaps reducing the gap in educational achievement between low- and high-SES students. Second, although speculative, these results may imply that Openness especially influences teachers' evaluations, rather than students' objective performance. Therefore, it may be recommendable to investigate more thoroughly how both SES and personality play a role in the formation of teachers' expectations.

This dissertation also suggests barriers for low-SES students in higher education. The finding that students from low-SES backgrounds who enter highly selective institutions in higher education experience a mismatch with the social environment, rather than with academic matters, may have implications for policy in higher education institutions. Policy interventions aimed at preventing dropout and promoting equal opportunities for low-SES students in highly selective institutions, could focus on these experiences of mismatch with the social environment. It is recommendable to consider whether highly selective institutions can do more to reduce these barriers for low-SES students. In addition, whereas undermatching is generally considered as undesirable because of the long-term consequences for job status and salary, this thesis shows both beneficial and unbeneficial 
consequences of undermatching during students' time in higher education. Undermatching may contribute to the social integration of low-SES students in higher education during their first year in higher education. A better social integration may in turn reduce the risk on college dropout in their early years in college. However, whereas undermatching seems to promote well-being with the social environment during their first year in higher education, on the longer term, undermatched students become less satisfied with both the academic and social environment than matched students. Therefore, counselors that advise and help students during the transition from secondary to tertiary education should be aware that undermatching might not help low-SES students on the longer term. Therefore, during the transition into higher education, a careful tradeoff has to be made between the advantages of undermatching (better integration during their first year in college and reduced risk of drop-out in the early phase of higher education) and the advantages of matching (more satisfaction and better academic match on the longer term).

\subsection{Future Research}

In order to promote fair testing and equal opportunities for students from all SESbackgrounds, the causal relation between context in math tests and math performance has to be investigated thoroughly. It is of importance to investigate this relation with an experimental design. In addition, more research is needed to explore the underlying mechanisms, such as stereotype threat (e.g., Schmader, Johns, \& Forbes, 2008), and narrowed attention and cognitive control (Mullainathan \& Sharif, 2013) leading to this bias.

Whereas the importance of personality in predicting educational outcomes is beyond discussion (Borghans, Duckworth, Heckman, \& Ter Weel, 2008; Heckman \& Kautz, 2012; Roberts, Kuncel, Shiner, Caspi, \& Goldberg, 2007), the malleability of personality traits is subject to debate (Roberts et al., 2017). In order to take advantage of the compensating role of Openness for background disadvantage, more insight is needed in the possibility to design interventions that enhance Openness, especially among low-SES students. In addition, it is of importance to investigate further whether students who display high levels of Openness are indeed evaluated as having higher academic potential by their teachers, especially when they have a low-SES background. Moreover, it is important to examine whether these evaluations are valid. In addition, more research is needed to investigate whether all facets of Openness, such as aesthetic sensitivity, or preference for novelty, are equally important in explaining the findings.

This thesis suggests that undermatching leads to more satisfaction during the first year in college, and, at the same time, to less satisfaction toward the later years in college. To investigate the long-term consequences of undermatching, future research should examine experiences after students' transition into the labour market. In addition, it is important to pay attention to other outcome variables than satisfaction and educational attainment as well, such as satisfaction with social relations with friends and family, and problem behavior such as delinquency and addiction. In particular, research on undermatching 
should take into account the recent findings in the literature with regard to harmful effects of upward social mobility on low-SES students' health (Brody, Yu, Miller, \& Chen, 2016; Miller, Yu, Chen, \& Brody, 2015; Wickrama, O’Neal, \& Lee, 2016). Investigating long-term effects of undermatching on a wide range of important life outcomes contributes to the understanding of development among socially mobile students, and to the development of policy and interventions to promote equal opportunities in education. 


\subsection{References}

Borghans, L., Duckworth, A. L., Heckman, J. J., \& Ter Weel, B. (2008). The economics and psychology of personality traits. Journal of Human Resources, 43(4), 972-1059.

Brody, G. H., Yu, T., Miller, G. E., \& Chen, E. (2016). Resilience in adolescence, health, and psychosocial outcomes, Pediatrics, 138(6), e20161042.

Ellis, B. J., Bianchi, J., Griskevicius, V., \& Frankenhuis, W. E. (2017). Beyond risk and protective factors: An adaptation-based approach to resilience. Perspectives on Psychological Science, 12(4), 561-587.

Jussim, L., Eccles, J., \& Madon, S. (1996). Social perception, social stereotypes, and teacher expectations: Accuracy and the quest for the powerful self-fulfilling prophecy. Advances in Experimental Social Psychology, 28, 281-388.

Mani, A., Mullainathan, S., Shafir, E., \& Zhao, J. (2013). Poverty impedes cognitive function. Science, 341, 976-980.

Miller, G. E., Yu, T., Chen, E., \& Brody, G. H. (2015). Self-control forecasts better psychosocial outcomes but faster epigenetic aging in low-SES youth. Proceedings of the National Academy of Sciences, 112(33), 10325-10330.

Mullainathan, S., \& Sharif, E. (2013). Scarcity: Why having too little means so much. London: Allen Lane.

Nelson, L. D., Simmons, J., \& Simonsohn, U. (2017). Psychology's renaissance. Annual Review of Psychology, 69, 511-534.

Roberts, B. W., Kuncel, N. R., Shiner, R., Caspi, A., \& Goldberg, L. R. (2007). The power of personality: The comparative validity of personality traits, socioeconomic status, and cognitive ability for predicting important life outcomes. Perspectives on Psychological science, 2(4), 313-345.

Roberts, B. W., Luo, J., Briley, D. A., Chow, P. I., Su, R., \& Hill, P. L. (2017). A systematic review of personality trait change through intervention. Psychological Bulletin, 143(2), 117.

Rogoff, B., Coppens, A. D., Alcalá, L., Aceves-Azuara, I., Ruvalcaba, O., López, A., \& Dayton, A. (2017). Noticing learners' strengths through cultural research. Perspectives on Psychological Science, 12, 876-888.

Schmader, T., Johns, M., \& Forbes, C. (2008). An integrated process model of stereotype threat effects on performance. Psychological review, 115(2), 336.

Sirin, S. R. (2005). Socioeconomic status and academic achievement: A meta-analytic review of research. Review of educational research, 75(3), 417-453.

Sternberg, R. J. (2005). The theory of successful intelligence. Interamerican Journal of Psychology, 39(2), 189-202.

Valencia, R. R. (2010). Dismantling contemporary deficit thinking: Educational thought and practice. New York, NY: Routledge.

Vohs, K. D. (2013). The poor's poor mental power. Science, 341, 969-970.

Wagenmakers, E. J., Wetzels, R., Borsboom, D., van der Maas, H. J. L., \& Kievit, R. A. (2012). An agenda for purely confirmatory research. Perspectives on Psychological Science, 7, 632-638.

Wickrama, K. A. S., O'Neal, C. W., \& Lee, T. K. (2016). The health impact of upward mobility: Does socioeconomic attainment make youth more vulnerable to stressful circumstances? Journal of Youth and Adolescence, 45(2), 271-285. 



\section{Acknowledgements}

Ik wil een aantal mensen in het bijzonder bedanken die een belangrijke rol hebben gespeeld in of tijdens de totstandkoming van dit proefschrift. Allereerst heel veel dank voor mijn promotor en co-promotor, die het mogelijk hebben gemaakt om dit traject met succes te doorlopen. Lex, wat een geluk dat ik jou heb leren kennen. Dankjewel voor de vele interessante gesprekken en voor het plezier als we samen over onderzoek nadenken. Ik wil je ook erg bedanken voor het vertrouwen dat je in me stelt en de kansen die je me biedt. Willem, heel erg bedankt voor alles wat je voor mij gedaan hebt. Onze meetings, de feedback en coaching zijn ontzettend belangrijk voor mij. Ik heb heel veel van je geleerd en ik ben heel dankbaar dat jij mijn copromotor wilde zijn.

Ook wil ik Trudie Schils bedanken voor de inspirerende en coachende gesprekken, in Maastricht, Toronto en Tübingen, met name in de laatste fase van het schrijven van dit proefschrift. Moreover, lots of thanks to Marion Spengler, for your help with our study on personality and SES. I really enjoy your expertise and our meetings. Thanks for all the valuable input and feedback.

Heel veel dank gaat ook uit naar Anja van den Broek. In de jaren dat je mijn leidinggevende was heb ik heel veel van jou geleerd. Ook heb je me geholpen door mij te steunen in de keuze om dit proefschrift te gaan schrijven, en door me op weg te helpen met data die nodig waren om mijn onderzoeksvragen te beantwoorden. Heel veel dank daarvoor. Mijn speciale dank gaat daarnaast ook uit naar een aantal mensen van wie ik tijdens mijn werk bij ResearchNed heel veel heb geleerd en/of mijn passie voor wetenschap hebben aangewakkerd en zo (misschien soms onbedoeld) belangrijk zijn geweest bij de totstandkoming van dit proefschrift. Bas Kurver, dankjewel voor alle gesprekken over sociologie en psychologie, en voor de geweldige analyse-vrijdagen. Jules Warps, veel dank voor je hulp met de Startmonitor-data. Ook wil ik Arjan Heijma, Maarten Wolbers en Louise Elffers bedanken voor de inspirerende samenwerkingen. Veel dank ook voor mijn collega's bij de Inspectie van het Onderwijs, Stef Böger, Karin van den Bosch, Grytsje Durkstra, Yvonne van den Beuken en natuurlijk Robert Tholen, voor de fijne samenwerking tijdens ons thema-onderzoek, dat samenviel met een belangrijke fase in mijn PhD traject. De sterke teamspirit was bijzonder en motiverend.

I also want to thank Gregory Wolniak. Dear Greg, it is great working with you. Thank you for the nice cooperation and the pleasant meetings. It inspires and motivates, in good times and in tough times. Moreover, I want to thank my dear colleagues from the Maastricht University. I really enjoy the shared passion for educational research. I feel lucky to work in a place with so many enthusiastic and inspiring researchers.

Lieve vrienden en familie, wat fijn dat jullie er zijn. In het bijzonder wil ik een paar mensen (in alfabetische volgorde) bedanken. Brigit, Carlijn, Diederik, Geertje, Jessica, Lisabeth, Martijn, Martijn, Marieke, Marieke, Marije, Nienke, Peggy, Rinke, Steffie, Willemijn en Yvonne, dank voor de fijne dagen, avonden en gezelligheid. Relaxen met jullie is een 
heel fijne tegenhanger van werken. Diederik, heel veel dank voor je hulp bij de kaft van dit proefschrift. In het bijzonder wil ik ook collega-moeder en vriendin Nienke bedanken voor de morele steun en alle gezellige momenten samen met onze gezinnen. Ook heel veel dank voor mijn paranimfen Willemijn en Lisabeth. Ik vind het heel bijzonder dat jullie me bij zullen staan bij de verdediging van dit proefschrift, na een vriendschap van vele jaren die begon tijdens ons eerste jaar aan de Radboud Universiteit.

Dear Solke, Thera, Doshin, Nico, Joanna, Milan, and Marta, thank you for your continuous moral support, and for the pleasant family gatherings that I deeply enjoy. Lieve Doshin, speciale dank voor al je hulp en voor je wijze ondersteuning. In het bijzonder wil ik ook tante Mieke bedanken voor de lieve aandacht en steun. Het is heel erg fijn dat je er altijd voor me bent. Oma, jou ben ik ook heel dankbaar. Terwijl je opgegroeid bent in een tijd waarin de wereld er heel anders uitzag, zeker ook wat betreft mogelijkheden voor vrouwen, heb ik me door jou altijd begrepen en gesteund gevoeld. Lieve Pierre, dank voor je continue liefdevolle ondersteuning en wijze raad. Pap, Coen, Marlou, dank voor jullie aandacht en ook voor het vertrouwen dat ik dit proefschrift tot een goed einde zou brengen. Veel dank gaat ook uit naar mijn moeder. Lieve mama, dankjewel voor de sterke, liefdevolle basis, en voor alles wat je voor mij gedaan hebt.

En natuurlijk ontzettend veel dank voor mijn heerlijke gezin. Dank voor de liefde, de gezelligheid, het avontuur, de ontspanning en de inspiratie. Bij jullie ben ik thuis. Lieve Harm, Tobias en Max, jullie betekenen alles voor mij. 


\section{Curriculum Vitae}

Marjolein Muskens was born in Nuenen, the Netherlands, on April 28, 1980. She graduated from high school (Gymnasium: Eckart College, REC) in Eindhoven in 1999. She attained a bachelor in clinical psychology in 2005, and graduated Cum Laude from the Research Master Behavioural Science in 2007 at the Radboud University. During her studies she worked as a research assistant at the Max Planck Institute for Psycholinguistic and at the Radboud University.

From 2008 to 2017, Marjolein worked as policy researcher at ResearchNed, a research institute specialized in social-scientific research, policy advice, and knowledge transfer in the overall field of education. As senior researcher, she conducted various studies by order of the Dutch Ministry of Education and the Dutch Inspectorate of Education. In 2015, Marjolein started as external Phd-student at Maastricht University, School of Business and Economics. Marjolein published her work in a variety of reports and journals, both academic and professional. She presented her research at various (inter)national conferences, among which (annual) meetings of the EAIR - The European Higher Education Society, the Dutch Educational Research Days (ORD), the American Educational Research Association (AERA), the International Sociological Association's RC28 (ISA RC28), and the Learning, Educational Achievement, and Life Course Development (LEAD) Research Network.

Since June 2017 Marjolein holds the position of postdoctoral researcher at the Department of Economics at Maastricht University, in the theme group "Learning and work", where she is involved in The Human Enhancement and Learning ( $\mathrm{HEaL}$ ) research programme, and the Educational Monitor Limburg (OML, Onderwijs Monitor Limburg). Marjolein is also member of the Developmental Evolutionary Ecological Psychology (DEEP) Lab at the Behavioural Science Institute (Radboud University). 




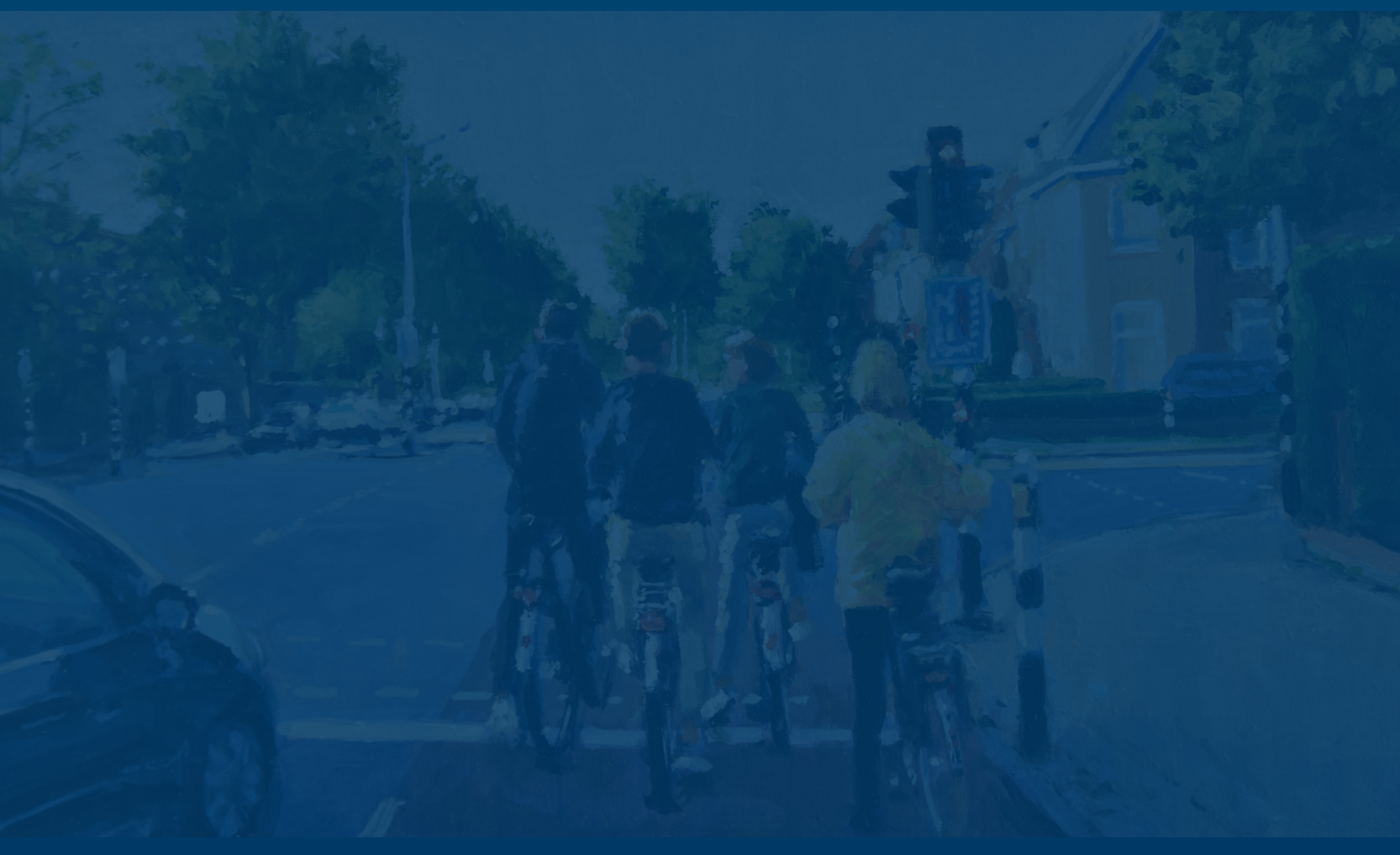

